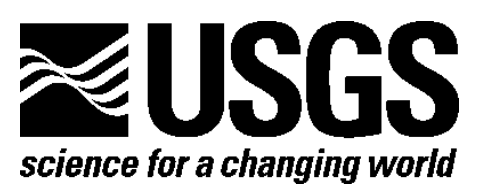

\title{
QRev-Software for Computation and Quality Assurance of Acoustic Doppler Current Profiler Moving-Boat Streamflow Measurements-Technical Manual for Version 2.8
}

By David S. Mueller

Open-File Report 2016-1068 


\section{U.S. Department of the Interior \\ SALLY JEWELL, Secretary}

\section{U.S. Geological Survey \\ Suzette M. Kimball, Director}

U.S. Geological Survey, Reston, Virginia: 2016

For more information on the USGS—-the Federal source for science about the Earth, its natural and living resources, natural hazards, and the environment-visit http://www.usgs.gov/ or call 1-888-ASK-USGS (1-888-275-8747).

For an overview of USGS information products, including maps, imagery, and publications, visit http://www.usgs.gov/pubprod/.

Any use of trade, firm, or product names is for descriptive purposes only and does not imply endorsement by the U.S. Government.

Although this information product, for the most part, is in the public domain, it also may contain copyrighted materials as noted in the text. Permission to reproduce copyrighted items must be secured from the copyright owner.

Suggested citation:

Mueller, D.S., 2016, QRev—Software for computation and quality assurance of acoustic Doppler current profiler moving-boat streamflow measurements-Technical manual for version 2.8: U.S. Geological Survey Open-File Report, 2016-1068, 79 p., http://dx.doi.org/10.3133/ofr20161068. 


\section{Contents}

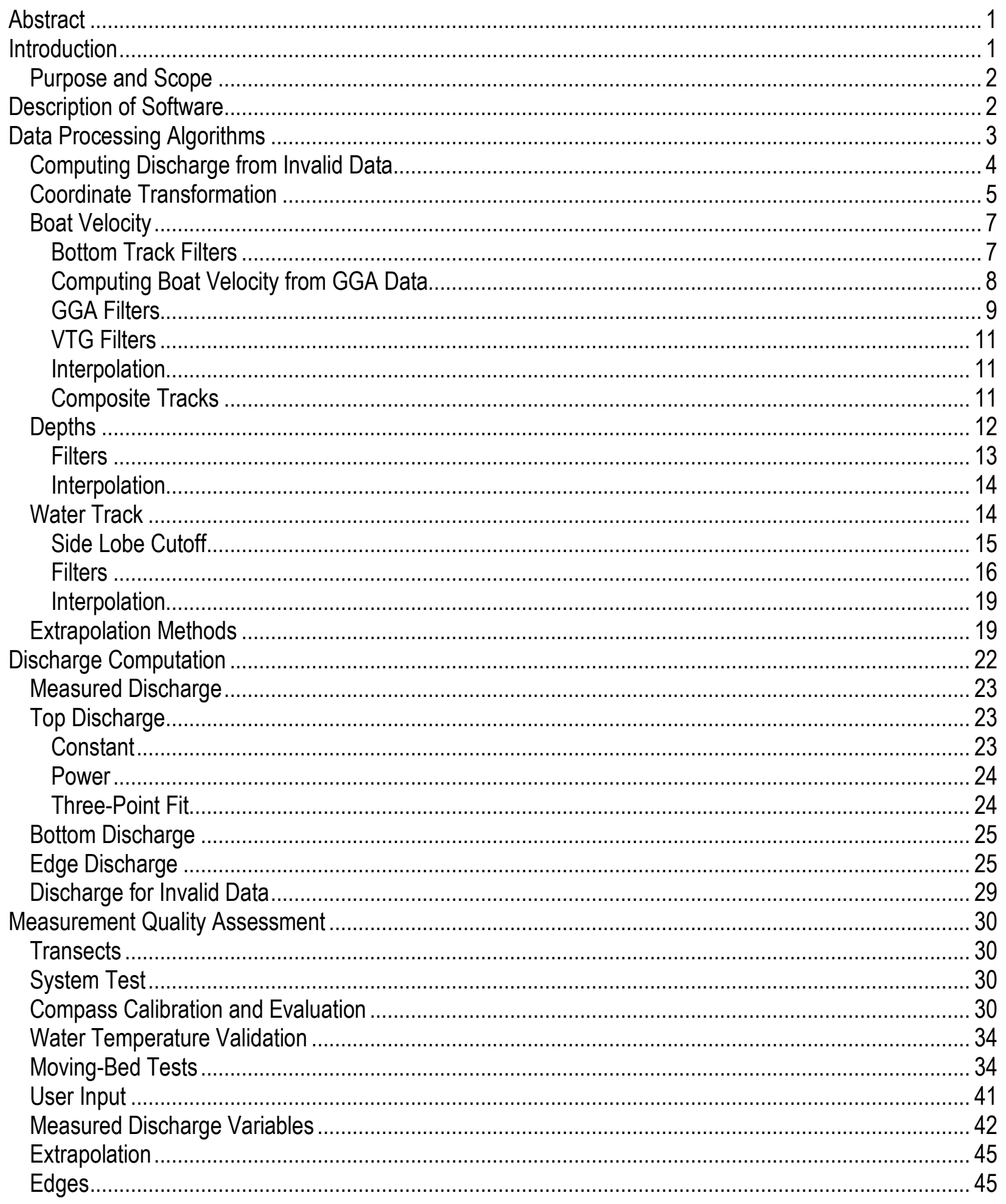




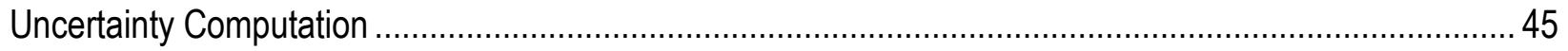

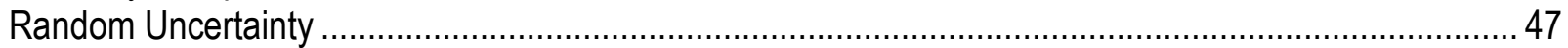

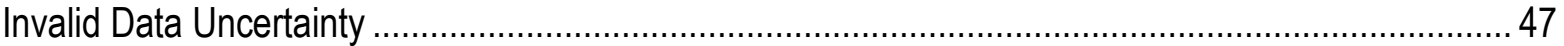

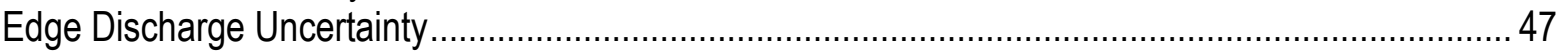

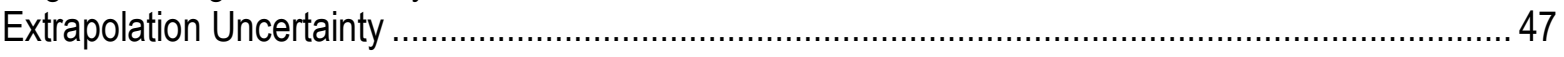

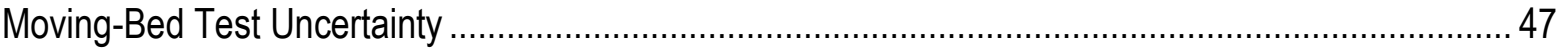

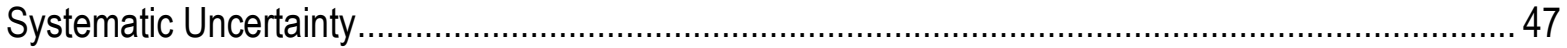

Estimated 95 Percent Uncertainty ........................................................................................... 47

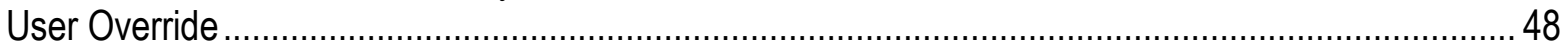

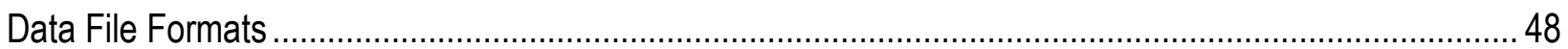

Summary and Need for Further Development .............................................................................. 48

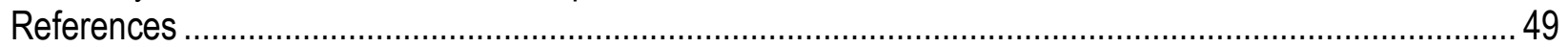

Appendix 1-Internal Data Format ......................................................................................... 51

Appendix 2-Extensible Markup Language (XML) Output Format ........................................................ 71

\section{Figures}

1. QRev main user interface .............................................................................................. 3

2. Acoustic Doppler current profiler beam pattern and locations of unmeasured areas in each

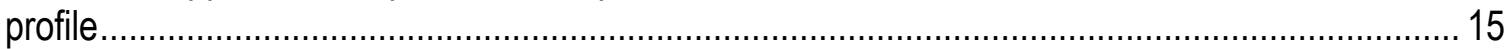

3. Example from RiverSurveyor Live of air entrainment partially blocking the acoustic signal in

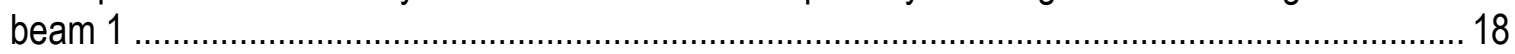

4. Illustration of measured and unmeasured zones of an acoustic Doppler current profiler

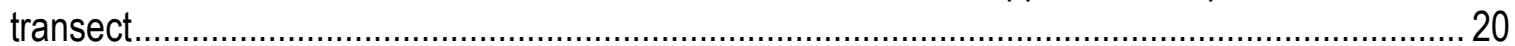

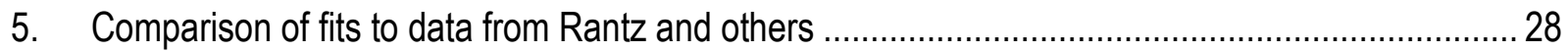

6. Comparison of proposed rectangular edge coefficients........................................................ 29

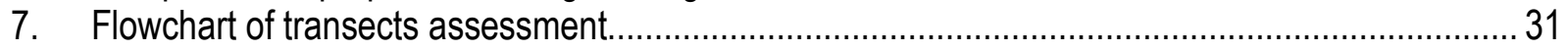

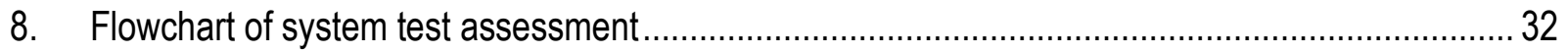

9. Flowchart of compass calibration and evaluation quality assessment ........................................33

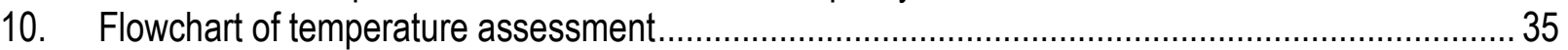

11. Flowchart of loop moving-bed test data quality evaluation .......................................................36

12. Flowchart of loop moving-bed test moving-bed evaluation ........................................................3

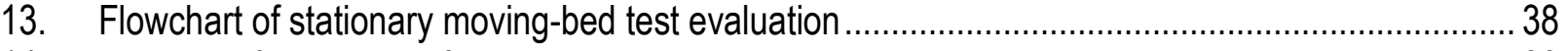

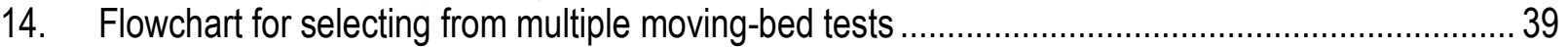

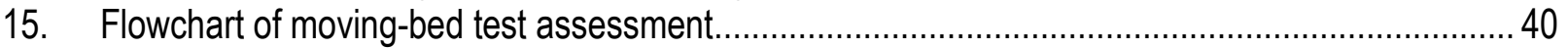

16. Flowchart of user input assessment .................................................................................. 41

17. Flowchart of measured discharge variable assessment .......................................................... 42

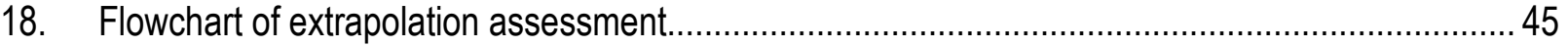

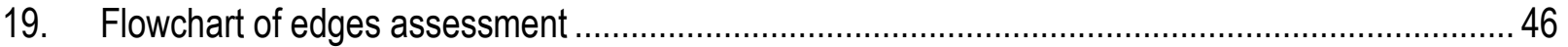




\section{Tables}

1. Summary of difference in software filter capabilities and handling of invalid data......................... 4

2. Progression of boat velocity reference in RiverSurveyor Live when composite tracks are

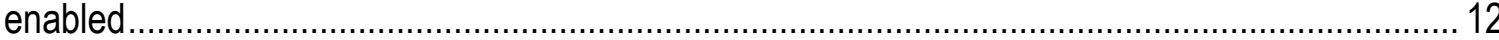

3. Progression of boat velocity reference in QRev when composite tracks are enabled.................... 12

4. QRev priority for composite depths................................................................................. 14

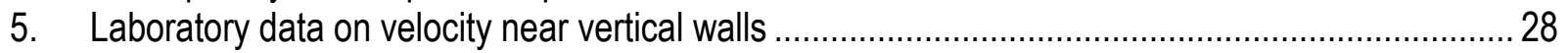

6. Summary of quality assessment messages for depth............................................................... 43

7. Summary of quality assessment messages for boat velocity ................................................... 43

8. Summary of quality assessment messages for water velocity .................................................. 44 


\section{Conversion Factors}

[International System of Units to U.S. customary units]

\begin{tabular}{|c|c|c|}
\hline Multiply & By & To obtain \\
\hline \multicolumn{3}{|c|}{ Length } \\
\hline meter $(\mathrm{m})$ & 3.281 & foot $(\mathrm{ft})$ \\
\hline meter $(\mathrm{m})$ & 1.094 & yard (yd) \\
\hline \multicolumn{3}{|c|}{ Flow rate } \\
\hline meter per second $(\mathrm{m} / \mathrm{s})$ & 3.281 & foot per second $(\mathrm{ft} / \mathrm{s})$ \\
\hline cubic meter per second $\left(\mathrm{m}^{3} / \mathrm{s}\right)$ & 35.31 & cubic foot per second $\left(\mathrm{ft}^{3} / \mathrm{s}\right)$ \\
\hline
\end{tabular}

Temperature in degrees Celsius $\left({ }^{\circ} \mathrm{C}\right)$ may be converted to degrees Fahrenheit $\left({ }^{\circ} \mathrm{F}\right)$ as follows:

$$
{ }^{\circ} \mathrm{F}=\left(1.8 \times{ }^{\circ} \mathrm{C}\right)+32 \text {. }
$$




\title{
QRev-Software for Computation and Quality Assurance of Acoustic Doppler Current Profiler Moving-Boat Streamflow Measurements-Technical Manual for Version 2.8
}

\author{
By David S. Mueller
}

\begin{abstract}
The software program, QRev applies common and consistent computational algorithms combined with automated filtering and quality assessment of the data to improve the quality and efficiency of streamflow measurements and helps ensure that U.S. Geological Survey streamflow measurements are consistent, accurate, and independent of the manufacturer of the instrument used to make the measurement. Software from different manufacturers uses different algorithms for various aspects of the data processing and discharge computation. The algorithms used by QRev to filter data, interpolate data, and compute discharge are documented and compared to the algorithms used in the manufacturers' software. QRev applies consistent algorithms and creates a data structure that is independent of the data source. QRev saves an extensible markup language (XML) file that can be imported into databases or electronic field notes software. This report is the technical manual for version 2.8 of QRev.
\end{abstract}

\section{Introduction}

The use of acoustic Doppler current profilers (ADCPs) from a moving boat is a commonly used method for measuring streamflow or discharge. The development of the ADCP has provided hydrographers and hydrologists with a tool that can substantially reduce the time for making streamflow measurements and has improved the safety of field personnel. These measurements have been reviewed and post-processed using manufacturer-supplied software. Using the manufacturer-supplied software, the review and processing depends on the user's knowledge and experience to interpret the quality of the measurement and set appropriate thresholds to screen out erroneous data. Software from different manufacturers use different algorithms for various aspects of the data processing and discharge computation. Consequently, if the same data set could be processed by each of the manufacturers' software, the resulting discharges would be different. Development of common and consistent computational algorithms combined with automated filtering and quality assessment of the data will provide substantial improvements in quality and efficiency of streamflow measurements and will ensure that U.S. Geological Survey (USGS) streamflow measurements are consistent, accurate, and independent of the manufacturer of the instrument used to make the measurement.

The USGS Office of Surface Water developed the computer program QRev that can be used to compute the discharge from a moving-boat ADCP measurement using data collected 
with any of the Teledyne RD Instruments (TRDI) or SonTek bottom tracking ADCPs. QRev applies consistent algorithms for the computation of discharge independent of the manufacturer of the ADCP. In addition, QRev automates filtering and quality checking of the collected data and provides feedback to the user of potential quality issues with the measurement. Various statistics and characteristics of the measurement, including a simple uncertainty assessment, are provided to assist the user in properly rating the measurement. QRev saves an extensible markup language (XML) file that can be combined with field notes collected in SVMobile to facilitate efficient loading of results into USGS databases.

\section{Purpose and Scope}

This report is the technical manual for version 2.8 of QRev. The purpose of this report is to document the algorithms used to process, filter, and interpolate the ADCP data; check the measurement for potential quality issues; compute discharge; and estimate the uncertainty of the measurement. Although QRev has the capability of applying the algorithms used in WinRiver II and RiverSurveyor Live, those methods have been documented by the manufacturer and will not be repeated herein, except where the same algorithm is used by default in QRev.

\section{Description of Software}

QRev was developed using Matlab and compiled for release in 2015 using Matlab version 2015b and compiler and runtime libraries version 9.0. Most of the code is written using object oriented programming techniques, although a few functions outside of an object class are also used. The general design of QRev was guided by the following criteria:

1. process SonTek and TRDI data,

2. use consistent algorithms,

3. use the best available data (interpolate only what is missing or invalid),

4. provide a logical workflow,

5. automate data quality assessment and feedback,

6. provide manual override for all automated filters,

7. use dialog windows with displays and output designed to evaluate specific problems,

8. provide uncertainty information to the user, and

9. use tablet friendly graphical user interface (GUI).

The GUI, shown in figure 1, is designed to be logical and tablet friendly. Processing steps, outlined by buttons, follow a logical top to bottom workflow. Logically, to load and review data the user would work from top to bottom along the buttons on the left. The approach leads the user through the premeasurement steps first. Depth, boat velocity, and water velocity are needed to compute discharge. Working from top to bottom, the best boat velocities are obtained first, then best depths, and finally the best water velocities, which are dependent on boat velocities and depths. Thus, the final discharge is based on the best boat velocities, depths, and water velocities that are available. 


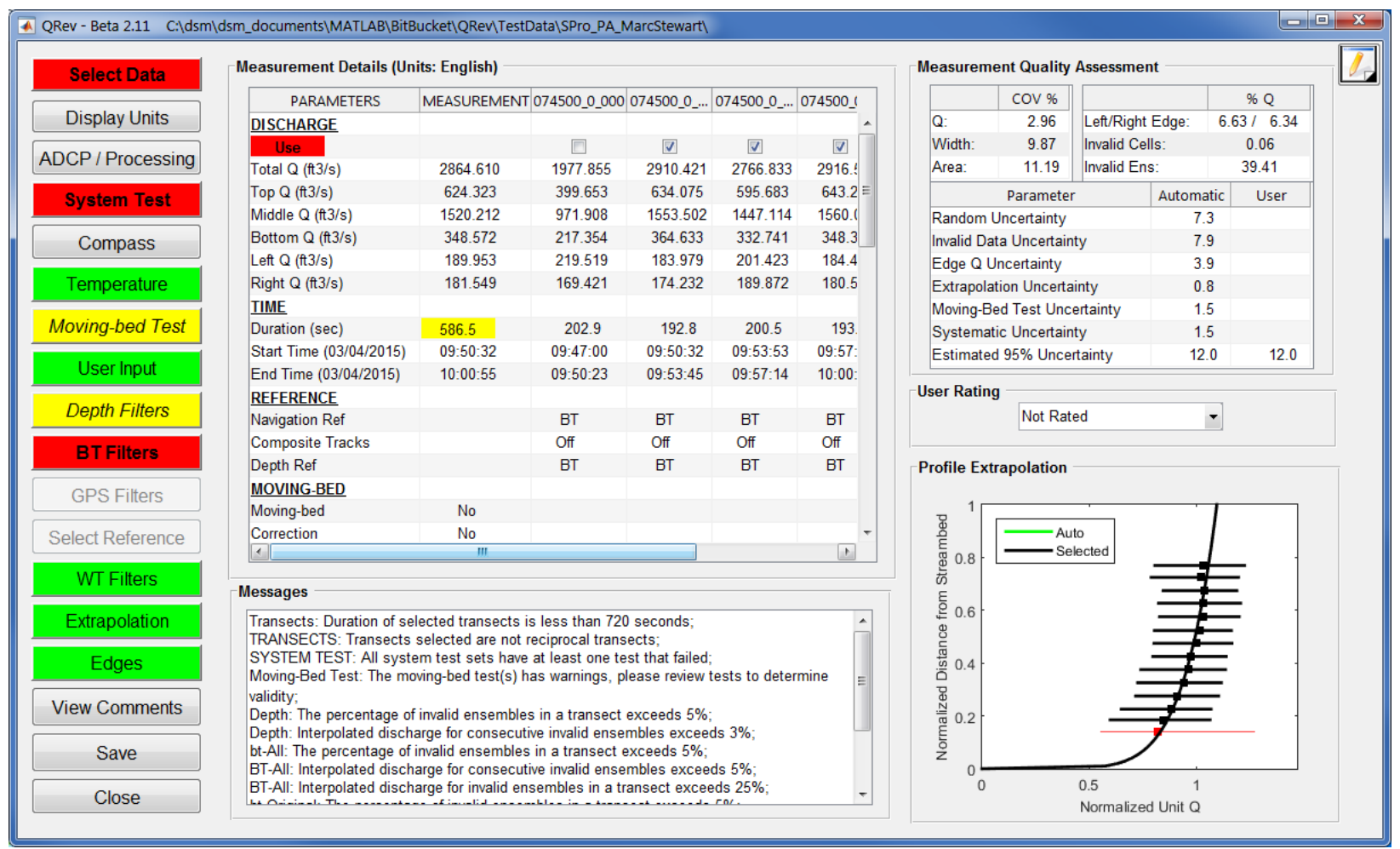

Figure 1. QRev main user interface. Although this window is labeled QREV Beta 2.11, the presentation is the same as in QREV version 2.8.

The buttons on the left will turn green, yellow, or red based on automated quality assessment criteria. If a button is yellow or red, an associated message in the message box will appear at the bottom of the main window. Buttons, check boxes, radio buttons, and dropdown menus are used in lieu of menus so that QRev is easier to use on a touch screen tablet. Each button opens a window that provides tables, text, options, and graphics needed to assess and process that particular aspect of the data. Details on the various windows are discussed in the Data Processing Algorithms section of this report and in the QRev User's Manual (Mueller, in press).

\section{Data Processing Algorithms}

Before velocity and depth data measured by the ADCP can be used to compute discharge, these data must be processed. The processing includes transforming the data into Earth coordinates, filtering the data to identify invalid data, and estimating the discharge in areas with invalid data. Invalid data could be identified by location, such as below the side lobe cutoff, or by a quality indicator, such as error velocity. Invalid data represent a spatial location within the cross section where valid data from which to compute discharge do not exist. Algorithms to estimate values for invalid data or the resulting discharge must be used to obtain the best possible measurement of discharge. This data processing step is where the manufacturers' software, WinRiver II and RiverSurveyor Live, differ the most. This step also is where QRev makes the most improvements in terms of accuracy and efficiency when compared to the current approaches used by the manufacturer. A summary of the differences in data processing among WinRiver II, RiverSurveyor Live, and QRev (as of July 2015) is listed in table 1. 
Table 1. Summary of difference in software filter capabilities and handling of invalid data.

\begin{tabular}{|c|c|c|c|}
\hline Type of processing & WinRiver II & RiverSurveyor Live & QRev \\
\hline \multicolumn{4}{|c|}{ Depths } \\
\hline Filter & $\begin{array}{l}\text { - Requires three valid } \\
\text { beams or valid vertical } \\
\text { beam } \\
\text { - Filters multiple } \\
\text { reflections } \\
\text { - Filters on valid bottom } \\
\text { track velocity }\end{array}$ & None & $\begin{array}{l}\text { - } \text { Filters multiple } \\
\text { reflections } \\
\text { - Outlier identification }\end{array}$ \\
\hline Invalid data & $\begin{array}{l}\text { Ensemble not used. } \\
\text { Duration of next valid } \\
\text { ensemble increased. }\end{array}$ & $\begin{array}{l}\text { Holds last valid depth } \\
\text { indefinitely. }\end{array}$ & $\begin{array}{l}\text { Linear interpolation of } \\
\text { each beam. }\end{array}$ \\
\hline \multicolumn{4}{|c|}{ Bottom track referenced boat velocity } \\
\hline Filter & $\begin{array}{l}\text { - Three-beam solutions } \\
\text { - Error velocity } \\
\text { - Vertical velocity }\end{array}$ & None & $\begin{array}{l}\text { - Three-beam solutions } \\
\text { - Error velocity } \\
\text { - Vertical velocity } \\
\text { - Outlier identification }\end{array}$ \\
\hline Invalid data & $\begin{array}{l}\text { Ensemble not used. } \\
\text { Duration of next valid } \\
\text { ensemble increased. }\end{array}$ & $\begin{array}{l}\text { Holds last valid velocity } \\
\text { for } 10 \text { ensembles then } \\
\text { sets velocity to zero. }\end{array}$ & Linear interpolation \\
\hline \multicolumn{4}{|c|}{ GPS referenced boat velocity } \\
\hline Filter & None & None & 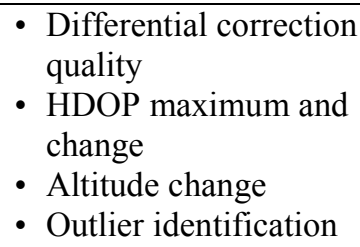 \\
\hline Invalid data & $\begin{array}{l}\text { Hold last valid } \\
\text { indefinitely. }\end{array}$ & $\begin{array}{l}\text { Ensemble not used. } \\
\text { Duration of next valid } \\
\text { ensemble increased. }\end{array}$ & Linear interpolation \\
\hline \multicolumn{4}{|c|}{ Water track velocity } \\
\hline Side lobe cutoff & $\begin{array}{l}\text { Uses slant and vertical } \\
\text { beam depths }\end{array}$ & Uses slant beams only & $\begin{array}{l}\text { Uses slant and vertical } \\
\text { beam depths }\end{array}$ \\
\hline Filter & $\begin{array}{l}\text { - Three-beam solutions } \\
\text { - Error velocity } \\
\text { - Vertical velocity }\end{array}$ & None & $\begin{array}{l}\text { - Three-beam solutions } \\
\text { - Error velocity } \\
\text { - Vertical velocity } \\
\text { - SNR filter (SonTek } \\
\text { data only) }\end{array}$ \\
\hline Invalid data & $\begin{array}{l}\text { Ensemble not used. } \\
\text { Duration of next valid } \\
\text { ensemble increased. }\end{array}$ & None & Linear interpolation \\
\hline
\end{tabular}

\section{Computing Discharge from Invalid Data}

WinRiver II provides the user the ability to filter data that appear to be erroneous by manually setting the parameters of several different types of filters. Properly setting these parameters requires some experience and may be an iterative process. WinRiver II also requires valid depth, boat velocity, and water velocity to compute discharge for an ensemble. If any of these three data types are not valid, the ensemble is considered invalid and the ensemble duration of the next valid ensemble is increased. The valid data in the invalid ensemble are effectively ignored, and the invalid ensemble is back filled with data from the next valid ensemble. Global 
Position System (GPS) data, however, are an exception; if a new GPS referenced velocity is not valid, the ensemble uses that last valid value.

RiverSurveyor Live does not provide the user with any tools to filter erroneous data. If the ADCP fails to obtain a valid depth in an ensemble, the previous valid depth is used until a new valid depth is collected. If the ADCP fails to obtain a valid bottom track referenced boat velocity for an ensemble, the previous valid boat velocity is used until a new valid boat velocity is obtain or until 10 ensembles have passed. After 10 consecutive ensembles with an invalid boat velocity, the boat velocity is set to zero for all subsequent ensembles until a valid boat velocity is obtained. This approach effectively computes of zero discharge for those ensembles assigned a zero boat velocity. If the boat velocity reference is set to GGA or VTG and the boat velocity is invalid, the data for that ensemble are ignored and the ensemble duration for the next valid ensemble is increased.

QRev uses the best available data and interpolates any invalid data. Computing discharge requires valid depth, boat velocity, and water velocity. Valid water velocity data require valid depth and valid boat velocity data. Boat velocity and depth data are independent. However, QRev uses transect length, which depends on boat velocity, to interpolate invalid depth data. Therefore, the workflow in QRev is to filter and interpolate the boat velocity so that every ensemble has a boat velocity (measured or estimated) associated with it prior to processing the depth data. The depth data are filtered and interpolated so that every ensemble has a depth. After every ensemble has boat velocities and depths, the water velocity data can be processed, filtered, and interpolated. The discharge for each ensemble can now be computed because each ensemble has a depth, boat velocity, and water velocity. All the valid data are used, and any invalid data are linearly interpolated.

\section{Coordinate Transformation}

Coordinate transformation is required to transform the raw data collected by the ADCP into orthogonal coordinates that are more convenient for the user. The Doppler shift is along (parallel to) the acoustic beams. Thus, the ADCP measures velocity vectors parallel to the four slant beams, which are typically referred to as beam velocity or beam coordinates. Those four vectors can be transformed into an orthogonal coordinate system, which is often referred to as instrument coordinates or $\mathrm{x}, \mathrm{y}$, and $\mathrm{z}$ coordinates ( $\mathrm{u}, \mathrm{v}$, and $\mathrm{w}$ for velocity components). Only three beams (vectors) are required to complete the transformation from beam coordinates to an orthogonal coordinate system.

For ADCPs with four beams, a fourth component can be computed. This fourth component is the difference in vertical velocity determined from each pair of opposing beams. SonTek refers to this fourth component as a difference velocity (SonTek, 2015), and TRDI scales the difference velocity and calls it an error velocity (Teledyne RD Instruments, 1998). The error velocity is scaled so that its magnitude (root-mean-square) matches the mean of the magnitudes of the horizontal velocity components ( $u$ and $v$ ). This scaling has been chosen so that in horizontally homogeneous flows, the variance of the error velocity will indicate the part of the variance of each of the horizontal components ( $u$ and $v$ ) attributable to instrument noise (Teledyne RD Instruments, 1998). If the flow field is homogeneous, the difference between these vertical velocities will average to zero. QRev applies this equation to data from TRDI and SonTek ADCPs and uses the equation to screen for invalid data. The following equations are used to transform beam coordinates to instrument coordinates for a four-beam ADCP: 


$$
\begin{gathered}
V_{y}=\left(B_{4}-B_{3}\right) /(2 \cos \theta), \\
V_{x}=\left(B_{1}-B_{2}\right) /(2 \sin \theta), \\
V_{z}=\left(B_{1}+B_{2}+B_{3}+B_{4}\right) /(4 \cos \theta), \text { and } \\
V_{\text {error }}=\left(B_{1}+B_{2}-B_{3}-B_{4}\right) /(2 \sqrt{2} \sin \theta)
\end{gathered}
$$

where

$$
\begin{array}{ll}
V_{y} & \text { is the streamwise velocity assuming beam } 3 \text { is pointed upstream, } \\
B_{4} & \text { is the radial velocity measured in beam 3, } \\
B_{3} & \text { is the radial velocity measured in beam } 4, \\
\theta & \text { is the tilt angle of the beams referenced to vertical, } \\
V_{x} & \text { is the cross-stream velocity assuming beam } 3 \text { is pointed upstream, } \\
B_{1} & \text { is the radial velocity measured in beam 1, } \\
B_{2} & \text { is the radial velocity measured in beam 2, } \\
V_{z} & \text { is the vertical velocity, and } \\
V_{\text {error }} & \text { is the scaled error velocity. }
\end{array}
$$

Note: Radial velocities are positive away from the transducer and negative towards the transducer. Expressing equations 1-4 in matrix form results in the nominal transformation matrix.

$$
\left[\begin{array}{cccc}
\frac{1}{2 \sin \theta} & \frac{-1}{2 \sin \theta} & 0 & 0 \\
0 & 0 & \frac{-1}{2 \sin \theta} & \frac{1}{2 \sin \theta} \\
\frac{1}{4 \cos \theta} & \frac{1}{4 \cos \theta} & \frac{1}{4 \cos \theta} & \frac{1}{4 \cos \theta} \\
\frac{1}{2 \sqrt{2} \sin \theta} & \frac{1}{2 \sqrt{2} \sin \theta} & \frac{-1}{2 \sqrt{2} \sin \theta} & \frac{-1}{2 \sqrt{2} \sin \theta}
\end{array}\right]
$$

The matrix is referred to as the nominal matrix because it is the perfect or ideal matrix. However, during manufacturing the transducers may not be potted perfectly resulting in some misalignment. The manufacturers test each ADCP and create a custom transformation matrix that accounts for the misalignment of the beams in each ADCP. QRev reads this custom matrix from the raw data, and if necessary, applies the matrix to the raw beam velocities.

The data are further transformed to correct for pitch and roll to produce what TRDI refers to as ship coordinates (Teledyne RD Instruments, 1998). An additional transformation to rotate the instrument coordinates for the heading measured by an internal or external compass, results in Earth or ENU (east, north, up) coordinates. QRev automatically applies the required transformations to convert the imported data to Earth coordinates. All computations in QRev are 
completed using Earth coordinates. For ADCPs that do not have a compass, the heading, pitch, and roll are set to zero and the transformation results in instrument coordinates.

If a four-beam ADCP only measures a valid Doppler shift on three beams, a three-beam solution for the $\mathrm{x}, \mathrm{y}$, and $\mathrm{z}$ velocity components is possible, but the error velocity cannot be computed. QRev uses the approach documented in Teledyne RD Instruments (1998) to compute $\mathrm{u}, \mathrm{v}$, and $\mathrm{w}$ using three beams. The invalid beam velocity is replaced with a value calculated from the last row of the instrument transformation matrix so as to force the error velocity to zero. The transformation from beam to instrument coordinates is then calculated in the usual way using the first three rows of the instrument transformation matrix.

\section{Boat Velocity}

To compute the water velocity, the velocity of the ADCP traversing the stream must be measured. Because the ADCP is typically deployed on a boat manned or tethered, the velocity of the ADCP is often referred to as the boat velocity. The boat velocity can be referenced to the bottom track, GGA, or VTG. QRev applies filters to each of these references to identify erroneous data. Erroneous data are marked invalid and are replaced with either a value from linear interpolation or from one of the other available boat velocity references if the composite tracks function is turned on. Although the filters for the various boat velocity references are different, the interpolation algorithm for invalid data is the same.

\section{Bottom Track Filters}

QRev includes the same filters used by WinRiver II: three-beam solutions, error velocity, and vertical velocity. Unlike WinRiver II where these filters must be set manually, QRev automatically determines the appropriate thresholds and applies the filters to data collected by either TRDI or SonTek ADCPs. In addition, QRev implements an outlier detection method.

\section{Beam Filter}

Current (2015) TRDI and SonTek ADCPs are based on a four-beam Janus configuration. Most bottom track velocity solutions will use all four beams, although only three beams are required to compute $\mathrm{u}, \mathrm{v}$, and $\mathrm{w}$ velocities in orthogonal coordinates $(\mathrm{x}, \mathrm{y}$, and $\mathrm{z}$ ). If one of the beams fails to provide a sufficient acoustic return for the Doppler shift, the boat velocity can be computed using the remaining three beams. In field applications, three-beam solutions can produce reliable velocities; however, the three-beam solution occasionally will be in obvious error. QRev provides the following three options for dealing with three- and four-beam solutions: (1) accept only four-beam solutions, (2) allow three-beam solutions, and (3) automatic mode, which is the default in QRev. In the automatic mode, the $u$ and $v$ components of the velocities associated with the three-beam solutions are compared to adjacent or nearby $u$ and $v$ components of the four-beam solution velocities. If both components of the three-beam velocities are within plus or minus (+/-) 50 percent of the respective components for the four-beam velocities, the three-beam velocity is assumed valid; conversely, if both components of the three-beam velocities are not within $+/-50$ percent of the respective components for the four-beam velocities, the three-beam velocity is marked invalid. 


\section{Error Velocity}

QRev applies equation 4 to data from TRDI and SonTek ADCPs and uses the equation to identify and mark erroneous data invalid. Horizontal velocities in an ensemble where the error velocities are outside the threshold are considered invalid. Like WinRiver II, QRev allows the user to manually set a threshold for error velocity or to turn error velocity filtering off. However, by default QRev computes this threshold automatically using a statistical measure of variance. Instrument noise associated with an ADCP is random and, thus, should follow a normal or Gaussian distribution. Therefore, using a measure of variance, outliers can be automatically identified. QRev uses +/- five times the interquartile range of all valid data as the automatic threshold. QRev computes the threshold, marks all outliers invalid, and repeats until no additional outliers are identified. Thus, only obvious outliers are identified and marked invalid.

Vertical Velocity

Typically, ADCPs deployed on a boat, manned or tethered, traversing a stream would have little or no vertical boat velocity relative to the streambed, except for that caused by wave action. On average, assuming that the stage of the stream is not changing, the vertical boat velocity averaged over a transect should be zero. The measured vertical boat velocity in each ensemble would be the result of wave action or instrument noise. Thus, like error velocity, the distribution of vertical boat velocities for a transect could be expected to be random. Deviations from a random distribution would suggest that the data are erroneous or that one of the assumptions is invalid. QRev applies the same approach to filtering vertical boat velocity as that described for error velocity.

\section{Other Outlier Identification Methods}

The boat velocity for a transect is a time series of velocities. The variation in velocity from one ensemble to the next is a function of the time between ensembles and boat operation. A best practice for measuring discharge with an ADCP from a moving boat is to operate the boat smoothly without sudden accelerations, decelerations, or turns (Mueller and others, 2013). Therefore, an algorithm that can smoothly fit the time series of boat velocities could be used to identify outliers or erroneous data. QRev uses a robust locally weighted scatterplot smooth (LOESS; Cleveland, 1979; Cleveland and Devlin, 1988) and a dynamically adjusted moving window with empirically derived settings to help identify outliers in boat speed. This filter is not applied automatically and defaults to off. Other algorithms that are more robust may be researched and included in future releases.

\section{Computing Boat Velocity from GGA Data}

The GGA sentence is defined by the NMEA 0183 standard (National Marine Electronics Association, 2002) and contains the time, horizontal position, differential quality, measure of horizontal dilution of precision, and altitude among other variables. The boat velocity is determined from the position in the GGA sentence by dividing the distance between successive positions by the time elapsed between those positions (differentiated position). To compute differentiated positions, the latitude and longitude must be transformed to $\mathrm{x}$ and $\mathrm{y}$ rectilinear components. The latitude and longitude could be transformed to $\mathrm{x}$ and $\mathrm{y}$ rectilinear components by computing the Universal Transverse Mercator (UTM) coordinates for the geographic positions; however, TRDI uses the following equations: 


$$
\begin{gathered}
\overline{L a t}=\left(\frac{\text { Lat }_{i}+\text { Lat }_{i-1}}{2}\right)\left(\frac{\pi}{180}\right), \\
\Delta x=\left(\frac{R_{e} \pi}{180}\right)\left(1+E \sin ^{2}\left(\overline{L^{2} t}\right)\right)\left(\operatorname{Lon}_{i}-\text { Lon }_{i-1}\right) \cos (\overline{\text { Lat }}), \\
\Delta y=\left(\frac{R_{e} \pi}{180}\right)\left(1-2 E+3 E \sin ^{2}(\overline{\text { Lat }})\right)\left(\text { Lat }_{i}-\text { Lat }_{i-1}\right), \\
u=\frac{\Delta x}{\Delta t}, \text { and } \\
v=\frac{\Delta y}{\Delta t}
\end{gathered}
$$

where

Lat $_{i}$ is the latitude for the most recent valid position;

Lat $_{i-1}$ is the latitude for the previous valid position;

$\Delta x \quad$ is the distance moved in the $\mathrm{x}$-direction in meters;

$R_{e} \quad$ is the radius of the Earth, 6,378,137 meters;

$E \quad$ is the ellipticity, $1 / 298.257223563$;

$\operatorname{Lon}_{i}$ is the longitude for the most recent valid position,

$L{ }_{i-1}$ is the longitude for the previous valid position;

$\Delta y \quad$ is the distance moved in the y-direction in meters;

$u \quad$ is the speed in the $\mathrm{x}$-direction in meters per second;

$\Delta t \quad$ is the time difference between the most recent and previous valid positions, seconds; and

$v \quad$ is the speed in the $\mathrm{y}$-direction in meters per second.

The results using UTM or equations 6-10 are similar but not exact for the UTM algorithms used in QRev. QRev is using the TRDI equations for consistency with previous measurements.

\section{GGA Filters}

Use of differentiated position requires accurate position solutions. The accuracy of the position data is affected by the differential correction applied to the data, the configuration of the satellites, and the path of the signal from the satellite to the GPS antenna (multipath). 


\section{Differential Correction Quality}

The GGA sentence contains a variable that indicates the quality or type of differential correction associated with the position. Although GPS receiver manufacturers have some differences, generally the quality is defined as follows:

- 0 , no position fix

- 1 , autonomous

- 2, differential correction

- 4, real-time kinematic

- 5, float real-time kinematic

QRev provides the user the option of accepting all data with a quality of one or greater, two or greater, and four or greater. The default is two or greater. Any position that does not meet or exceed the quality setting is marked invalid.

\section{Altitude Filter}

Data collected from a boat traversing a stream should have a nearly constant altitude, excepting vertical movement because of wave action, which for inland waterways is typically minimal. Thus, the altitude measured from the GPS receiver on the boat should be nearly constant. The altitude measured by a GPS receiver is typically about three times less accurate than the horizontal position (http://water.usgs.gov/osw/gps/). Thus, for a GPS receiver with submeter differentially corrected accuracy, the vertical accuracy would be about 3 meters $(9.8$ feet). The GPS tabular view in WinRiver II turns the altitude field red if the altitude changes by more than 3.5 meters (11.5 feet) during a transect, but the data are not marked invalid (Teledyne RD Instruments, 2014). QRev provides the ability to mark GPS data invalid based on the change in altitude during a transect. The altitude filter in QRev defaults to an automatic setting and uses a threshold of $+/-3$ meters from the mean of valid altitudes in the transect. The mean altitude for all valid altitudes is computed, altitudes exceeding the filter are marked invalid, and the process is repeated until all valid altitudes are within the threshold. The user can change the filter setting to manual and adjust the threshold to any desired value or turn the filter off.

\section{Horizontal Dilution of Precision (HDOP)}

Dilution of precision is a term used to characterize the geometric configuration of the satellites and its effect on the accuracy of the GPS position fix. The lower the dilution of precision, the better the accuracy of the position fix. Position dilution of precision is commonly used and provides an overall rating of precision of horizontal and vertical positions. However, only the horizontal position accuracy generally is of interest for boat velocity reference, and the horizontal dilution of precision (HDOP) is available in the GGA sentence.

WinRiver II displays the HDOP and change in HDOP during a transect in the GPS tabular view. These values are red if the HDOP exceeds two or the change in HDOP within a transect is greater than one. QRev expands on this approach by using HDOP as a filter to mark GPS data invalid. The QRev filter has two parts: (1) a threshold on the value of HDOP, which defaults to two, and (2) a threshold on the change in HDOP, which defaults to one. The filter marks all data with an HDOP value greater than the HDOP threshold invalid. The mean HDOP for the transect is then computed using the valid data, and all data with a deviation from the mean greater than the change threshold are marked invalid. The process is repeated until all valid 
data meet the filter criteria. The user can change the filter to manual and adjust the HDOP and the change in HDOP thresholds or turn the filter off.

\section{Other Outlier Identification Methods}

The same outlier identification methods discussed in the "Bottom Track Filters" section can be applied to boat velocities referenced to GGA. This filter is not applied automatically and defaults to off.

\section{VTG Filters}

The velocity reported in the NMEA VTG sentence contains only a velocity and direction. The velocity is typically based on measured Doppler shifts in the satellite signals, but some receivers may use differentiation of successive positions to compute the reported velocity. Wagner and Mueller (2011) determined that VTG can be a valid alternative for measuring discharge with an ADCP in moving-bed environments without the assistance of differential corrections; however, VTG-based discharges may be inaccurate, particularly for boat speeds less than about 0.8 foot per second. Use of the Doppler shift to determine velocity does not require and is unaffected by differential corrections. This velocity measurement can be robust because it is resistant to some of the errors that are problematic for position determination, such as multipath errors.

\section{Horizontal Dilution of Precision (HDOP)}

The geometry of the satellites affects the accuracy of the velocity measured by the Doppler shift. The VTG sentence does not provide any data quality parameters. If the GGA sentence is available, QRev applies the same HDOP filtering to VTG data as described for GGA data.

\section{Other Outlier Identification Methods}

The same outlier identification methods discussed in the "Bottom Track Filters" section can be applied to boat velocities referenced to VTG. This filter is not applied automatically and defaults to off.

Interpolation

Linear interpolation is used to estimate boat velocities that have been determined to be invalid. The same interpolation algorithm is used for boat velocities referenced to bottom track, GGA, and VTG. Linear interpolation is applied to each velocity component ( $u$ and v) with ensemble time as the independent variable. QRev maintains a record of the source of the velocity so that those velocities that have been interpolated are easily identified.

\section{Composite Tracks}

Composite tracks allows use of one of the other available boat velocity references to replace invalid data in the primary boat velocity data. RiverSurveyor Live implements composite tracks as listed in table 2. QRev implements a simpler progression as listed in table 3. A more complex scheme that includes the quality of the differential correction, as used by RiverSurveyor 
Live, or that includes some combining of all available sources like a Kalman filter may be considered for future development.

Table 2. Progression of boat velocity reference in RiverSurveyor Live when composite tracks are enabled (D.S. Mueller, U.S. Geological Survey, written commun., 2008).

[BT, bottom track; RTK, real-time kinematic]

\begin{tabular}{|c|c|}
\hline Reference quality & Reference used \\
\hline \multicolumn{2}{|l|}{ BT preferred } \\
\hline BT valid & BT \\
\hline BT invalid & RTK GGA \\
\hline BT invalid, RTK GGA invalid & VTG \\
\hline BT invalid, RTK GGA invalid, VTG invalid & Invalid data \\
\hline \multicolumn{2}{|l|}{ GGA preferred } \\
\hline RTK GGA valid & RTK GGA \\
\hline RTK GGA invalid & GGA \\
\hline RTK GGA invalid, DGPS invalid & VTG \\
\hline RTK GGA invalid, DGPS invalid, VTG invalid & BT \\
\hline RTK GGA invalid, DGPS invalid, VTG invalid, BT invalid & Invalid data \\
\hline \multicolumn{2}{|l|}{ VTG preferred } \\
\hline VTG valid & VTG \\
\hline VTG invalid & BT \\
\hline VTG invalid, BT invalid & Invalid data \\
\hline
\end{tabular}

Table 3. Progression of boat velocity reference in QRev when composite tracks are enabled.

[BT, bottom track; RTK, real-time kinematic]

\begin{tabular}{|c|c|}
\hline Reference quality & Reference used \\
\hline \multicolumn{2}{|c|}{ BT preferred } \\
\hline BT valid & BT \\
\hline BT invalid & VTG \\
\hline BT invalid, VTG invalid & GGA \\
\hline BT invalid, VTG invalid, GGA invalid & Interpolate from BT data \\
\hline \multicolumn{2}{|c|}{ GGA preferred } \\
\hline GGA valid & GGA \\
\hline GGA invalid & VTG \\
\hline GGA invalid, VTG invalid & BT \\
\hline GGA invalid, VTG invalid, BT invalid & Interpolate from GGA data \\
\hline \multicolumn{2}{|c|}{ VTG preferred } \\
\hline VTG valid & VTG \\
\hline VTG invalid & GGA \\
\hline VTG invalid, GGA invalid & BT \\
\hline VTG invalid, GGA invalid, BT invalid & Interpolate from VTG data \\
\hline
\end{tabular}

\section{Depths}

The depth from water surface to streambed used to compute discharge is determined from the draft of the ADCP and either from the average of the four beam depths from the four slant beams (referred to hereafter as BT) or from the depth from the vertical beam (VB) for those ADCPs equipped with a vertical beam. The draft or transducer depth is measured and entered in the data collection software (WinRiver II or RiverSurveyor Live) by the user. QRev allows the user to edit the draft if an error was made in the original entry. If the ADCP is equipped with a 
vertical beam, the user can select to use the VB depth, the BT depth, or a composite depth in the manufacturers' software or in QRev. How the BT depth is computed from the four slant beams and how composite depths are implemented are different in the manufacturers' software and QRev.

The manufacturers and QRev use different methods to compute the vertical depth from the slant beams. Both methods adjust the slant range for the designed angle of the beams from the vertical. SonTek also corrects the depths measured by the slant beams and vertical beam for pitch and roll, but TRDI does not (SonTek, 2015; Teledyne RD Instruments, 2007). QRev uses the vertical depths provided by the manufacturers. Implementing pitch and roll compensation for the measured depths is being considered for a future version. A preliminary assessment indicated about a 0.4 percent change in average depth for a mean pitch or roll of 5 degrees.

The method used to compute the BT depth from the four slant beams is also different between the manufacturers. SonTek uses a simple numerical average of the four beam depths. TRDI has the option of a simple numerical average, but the default is an inverse depth weighted (IDW) average (SonTek, 2015; Teledyne RD Instruments, 2014). QRev uses the IDW average computed using the following equations:

$$
\begin{gathered}
D_{T_{i}}=D_{i}-D_{D}, \\
w_{i}=1-\frac{D_{T_{i}}}{\sum D_{T_{i}}}, \text { and } \\
\bar{D}=D_{D}+\frac{\sum D_{T_{i}} w_{i}}{\sum w_{i}}
\end{gathered}
$$

where

$D_{T_{i}} \quad$ is the depth from the transducer to the streambed for the i-th beam,

$D_{i} \quad$ is the vertical depth for the $\mathrm{i}$-th beam,

$D_{D} \quad$ is the depth of the transducer,

$w_{i} \quad$ is the weight computed for the $\mathrm{i}$-th beam, and

$\bar{D} \quad$ is the average depth (BT) for an ensemble.

\section{Filters}

Depths from individual beams, whether slant beams or the vertical beam, occasionally contain spikes in the measured depth. These spikes may be shallow and caused by aquatic life or by debris somewhere in the water column. These spikes may also be deep and caused by the ADCP digitizing the multiple of the acoustic return. RiverSurveyor Live does not provide any filtering of the depths. WinRiver II has several options for filtering the depth data from the slant beams. To screen spikes, WinRiver II uses an algorithm that marks a beam invalid if it is more than 75 percent different from the other beams (Teledyne RD Instruments, 2014). WinRiver II also requires at least three valid beam depths or the average depth for the ensemble is considered invalid. WinRiver II also has an option to mark all depths invalid that are not associated with a valid bottom track velocity (Teledyne RD Instruments, 2014). 
QRev uses a similar 75 percent criteria to identify spikes in the slant beams. The ratio of each beam to the other beams is computed, and if the ratio exceeds 1.75 , the beam in the numerator is marked invalid. This method is intended to filter out depths that are too deep because of the ADCP digitizing the second reflection rather than the first reflection. If used indiscriminately, this filter will tend to bias the depth to the shallower depths. This filter is not applied automatically and defaults to off.

QRev also uses an outlier identification method using a robust LOESS smooth similar to that described in the "Boat Velocity" section. Like the boat velocity, the depths from each beam form time series of depths. Most cross sections vary gradually and sudden deviations from the trend are indicative of erroneous values. Therefore, an algorithm that can smoothly fit the time series of depths for each beam could be used to identify outliers or erroneous data. QRev uses a robust LOESS smooth and a dynamically adjusted moving window with empirically derived settings to help identify outliers. This filter is applied automatically but may be turned off by the user. Other algorithms that are more robust may be researched and included in future releases.

Interpolation

QRev uses linear interpolation to estimate beam depths for any data determined to be invalid. The linear interpolation is applied to each beam prior to computing the average depth for an ensemble. The independent variable in the linear interpolation is the cumulative length along the transect path.

\section{Composite Depths}

Composite depths allows the use of the depth from a secondary depth source when the depth from the primary source is invalid. Composite depths is available for TRDI ADCPs equipped with a vertical beam (RiverRay and RiverPro). TRDI's implementation gives first preference to the vertical beam, then to the depth sounder (if available), and then to the average of the four slant beams (Teledyne RD Instruments, 2014). This setting is the default setting in WinRiver II. SonTek's implementation of composite depths does not support an external depth sounder and allows the user to select the primary reference (average of the four slant beams or the vertical beam) and sets the other as the secondary reference (SonTek, 2015). QRev extends the manufacturers' implementations to support user selection of the primary reference and to include the external depth sounder and linearly interpolated depths as other options (table 4). If data from an external depth sounder are available, QRev assumes that the user had a good reason for collecting data with the depth sounder; therefore, depth sounder data (if not selected as primary) are assumed as the first option. If none of the available depth sources provide a valid depth, QRev will use a value determined from linear interpolation of the primary depth source.

Table 4. QRev priority for composite depths.

\begin{tabular}{|l|l|l|l|}
\hline Primary & BT & VB & DS \\
\hline First option & DS & DS & VB \\
\hline Second option & VB & BT & BT \\
\hline Third option & Interpolated BT & Interpolated VB & Interpolated DS \\
\hline
\end{tabular}

\section{Water Track}

Water track data can only be processed after valid depths and boat velocities have been determined. The valid depths are used to determine the location of the side lobe cutoff. The boat 
velocity is used to compute a water velocity relative to a fixed reference rather than a moving boat. Finally, the water velocities are filtered and invalid data interpolated.

\section{Side Lobe Cutoff}

An ADCP cannot measure the water velocity near the streambed because of side lobe interference (fig. 2). Most transducers that are developed using current (2015) technology emit parasitic side lobes off of the main acoustic beam. The magnitude of the energy in a side lobe reflected from the streambed is sufficiently close to the magnitude of energy in the main beam reflected from scatterers in the water column to cause potential errors in the measured Doppler shift. The portion of the water column near the streambed affected by this side lobe interference varies from 6 percent for a 20-degree system to 13 percent for a 30-degree system and can be computed as follows:

$$
D_{S L}=D(1-\cos (\theta))
$$

where

$D_{S L} \quad$ is the distance from the streambed affected by side lobe interference,

$D \quad$ is the distance from the transducer to the streambed, and

$\theta \quad$ is the angle of the transducers from the vertical (Mueller and others, 2013).

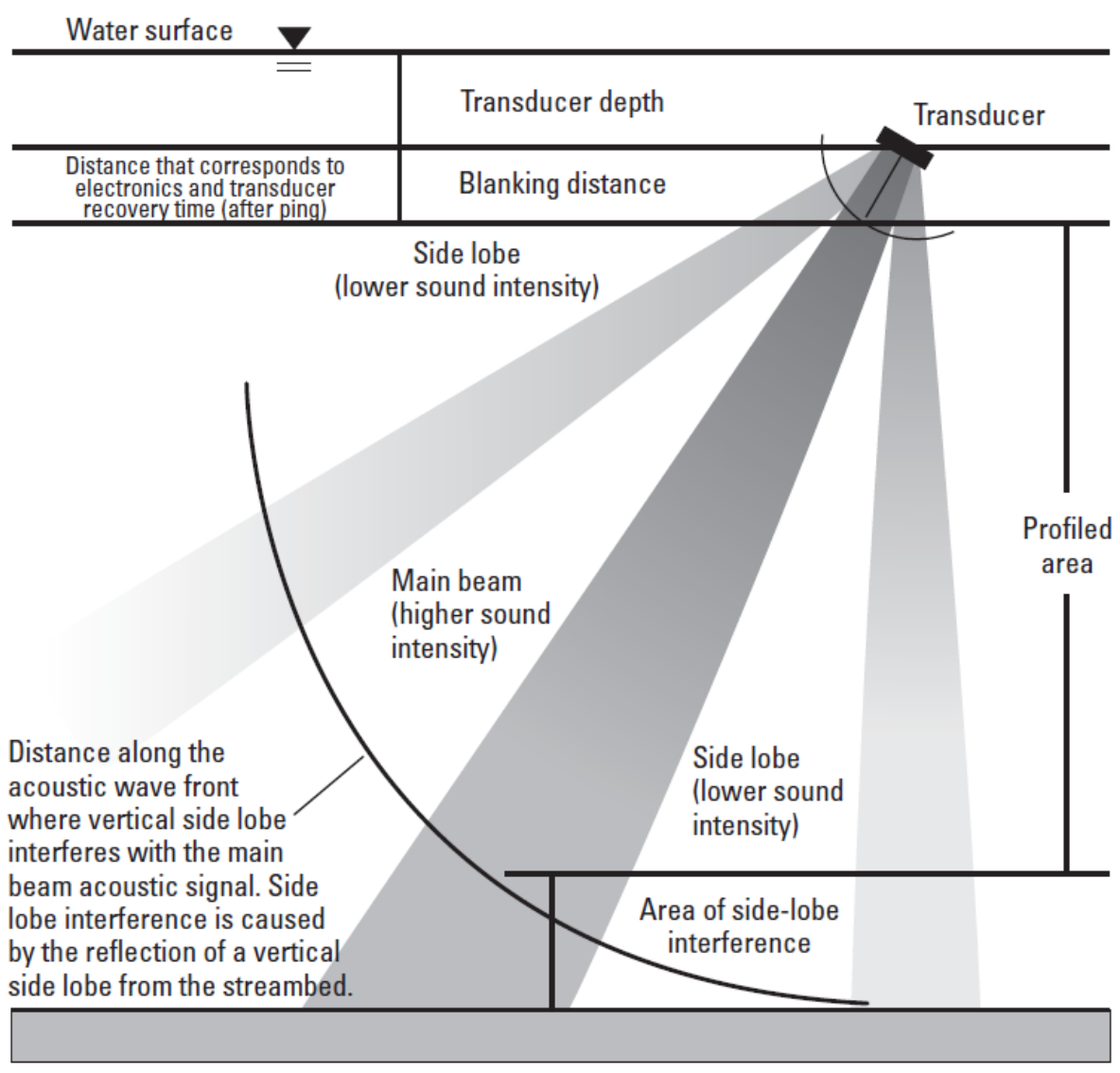

Figure 2. Acoustic Doppler current profiler beam pattern and locations of unmeasured areas in each profile (from Simpson, 2002). 
The distance from the streambed potentially affected by the side lobe is computed in equation 14; however, to determine the last valid depth cell in a profile requires the depth cell size, transmit length, and lag between transmit pulses to be accounted for. TRDI determines the last valid depth cell for standard modes (Teledyne RD Instruments, 2014) as follows:

$$
D_{B C O}=\left(\left(D_{\min }-\mathrm{D}_{A D C P}\right) \cos (\theta)+\mathrm{D}_{A D C P}\right)-\left(\left(D_{L}+D_{T}+D_{B}\right) / 2\right)
$$

where

$D_{B C O}$ is the cutoff for the center line of the deepest depth cell,

$D_{\min }$ is the minimum vertical depth of the four beams,

$D_{A D C P}$ is the depth of the transducers below the water surface (draft),

$D_{L} \quad$ is the lag between transmit pulses or correlation lag,

$D_{T} \quad$ is the transmit pulse length, and

$D_{B} \quad$ is the depth cells size.

For TRDI pulse coherent modes, the lag is equal to the depth and, thus, is not considered in the cutoff computation and equation 15 becomes,

$$
D_{B C O}=\left(\left(D_{\min }-\mathrm{D}_{A D C P}\right) \cos (\theta)+\mathrm{D}_{A D C P}\right)-\left(\left(D_{T}+D_{B}\right) / 2\right)
$$

For TRDI ADCPs with a vertical beam, if the vertical beam is selected as the primary depth reference or used in composite depths the $\mathrm{D}_{\min }$ is the minimum depth of all five beams.

SonTek's algorithms for handling the side lobe cutoff for RiverSurveyor ADCPs (Lyn Harris, SonTek, written commun., 2014) can be restated in the form of equation 16. SonTek also replaces the $\cos (\theta)$ with a user defined percentage that defaults to 10 percent.

$$
D_{B C O}=\left(\left(D_{\min }-\mathrm{D}_{A D C P}\right)\left(1-C_{S L}\right)+\mathrm{D}_{A D C P}\right)-\left(\left(D_{T}+D_{B}\right) / 2\right)
$$

where

$C_{S L}$ is the user defined percentage.

QRev uses the same approach as the manufacturers in handling the side lobe when the beams are valid. If none of the beams have a valid depth, QRev uses the mean depth computed from the linear interpolation as $D_{\min }$. If one or more of the beams are invalid, the side lobe cutoff is computed based on the mean depth using the interpolated and valid beam depths and is compared to the side lobe cutoff using the minimum of the valid beams. If the side lobe cutoff is shallower using the mean depth, the side lobe cutoff is adjusted. Only bins with a center line depth less than $D_{B C O}$, computed using equations $15-17$, are used to compute discharge. SonTek and TRDI allow the user to specify additional bins above the side lobe to be marked invalid. This feature is not supported in QRev but will be considered for a future release.

\section{Filters}

QRev includes the same water track filters used by WinRiver II-three-beam solutions, error velocity, and vertical velocity and in addition, a signal-to-noise ratio (SNR) filter. QRev defaults to an automated application of these filters. The three-beam solutions, error velocity, and vertical velocity filters are applied to the water velocity in individual depth cells. The SNR filter is only applied to SonTek data and is applied to an ensemble. 


\section{Beam Filter}

Current (2015) TRDI and SonTek ADCPs are based on a four-beam Janus configuration. Most water track velocity solutions will use all four beams, although only three beams are required to compute $\mathrm{u}, \mathrm{v}$, and $\mathrm{w}$ velocities in orthogonal coordinates ( $\mathrm{x}, \mathrm{y}$, and $\mathrm{z}$ ). If one of the beams fails to provide a sufficient acoustic return for the Doppler shift, the velocity can be computed using the remaining three beams. In field applications, three-beam solutions can produce reliable velocities; however, the three-beam solution occasionally will be in obvious error. QRev provides the following three options for dealing with three- and four-beam solutions: (1) accept only four-beam solutions, (2) allow three-beam solutions, and (3) automatic mode (default). In the automatic mode, QRev identifies all three-beam solutions. The $\mathrm{u}$ and $\mathrm{v}$ components of the velocities associated with the three-beam solutions are estimated from linear regression of adjacent or nearby $u$ and $v$ components of the four-beam solution velocities. If both components of the measured three-beam velocities are within $+/-50$ percent of the estimated velocities from linear regression, the three-beam velocity is assumed valid, if not, the three-beam velocity is marked invalid.

\section{Error Velocity}

QRev applies equation 4 to water velocities in individual depth cells for TRDI and SonTek data and uses the equation to identify and mark erroneous data invalid. Horizontal velocities in an depth cell where the error velocities are outside the threshold are considered invalid. Like WinRiver II, QRev allows the user to manually set a threshold for error velocity or to turn error velocity filtering off. However, by default, QRev computes this threshold automatically using a statistical measure of variance. Instrument noise associated with an ADCP is random and, thus, should follow a normal or Gaussian distribution. Therefore, using a measure of variance, outliers can be automatically identified. QRev uses +/- five times the interquartile range of all valid data as the automatic threshold for determining outliers. QRev computes the threshold, marks all outliers invalid, and repeats the process until no additional outliers are identified. Thus, only obvious outliers are identified and marked invalid.

\section{Vertical Velocity}

Generally, the vertical velocities in a cross section would average to zero; however, depending on channel configuration and proximity to hydraulic structures, this assumption may not be valid. Because vertical velocities in a cross section cannot be assumed to average to zero, filtering on vertical water velocities is more difficult than filtering on error velocities. Despite this complexity, experience has indicated that application of the same filtering approach used with error velocities is generally effective for vertical velocities. QRev applies an automatic filter using the same approach as the error velocity filter to vertical velocities. If significant real variations exist in the vertical velocity, the data may be better represented by turning off the vertical filter.

\section{Flow Disturbance}

The immersion of the ADCP into the flowing water and the boat and mount used to deploy the ADCP will create a disturbed flow field around the ADCP that no longer represents the free stream velocities (Mueller and others, 2007). Model and field data comparisons have determined that the default blanking distance for most ADCPs is adequate to block data 
collection in the disturbed region (Mueller, 2015). The SonTek RiverSurveyor M9 is the exception. The USGS policy is that data in the range from 0 to 0.16 meter from the transducer be considered invalid (Office of Surface Water, 2013). QRev automatically enforces this policy for data collected with a SonTek RiverSurveyor M9 by setting the excluded distance to 0.16 meter. If a screening distance is set in RiverSurveyor Live such that the excluded distance is greater than 0.16 meter, that greater distance is used by QRev; however, if the screening distance in RiverSurveyor Live is set such that the excluded distance is less than 0.16 meter, QRev will set the exclude distance to 0.16 meter. The excluded distance is measured from the transducer face and can be edited by the user. The excluded distance is set to zero for all other ADCPs.

\section{Signal-to-Noise Ratio Filter}

Air entrainment beneath the SonTek RiverSurveyor ADCPs can cause the effective beam angles to deviate from the actual angles, and experience has indicated that this deviation often results in measured velocities and discharge that are biased low. The air entrainment can be identified by a separation of the beams in a profile plot of the beams SNR (fig. 3). Empirical analysis of numerous measurements made with a SonTek RiverSurveyor M9 resulted in a simple filter to identify ensembles with potential air entrainment. The filter computes the mean SNR for each beam in each ensemble using SNR data from depth cells below the excluded distance and above the side lobe cutoff. The SNR range is computed as the difference in SNR for the beams with the minimum and maximum mean SNR in each ensemble. Any ensemble with a range greater than 12 decibels is assumed to be affected by air entrainment and the velocities are marked invalid. This filter is only applied to data collected with SonTek ADCPs. Broader application of the filter will be considered for future releases.

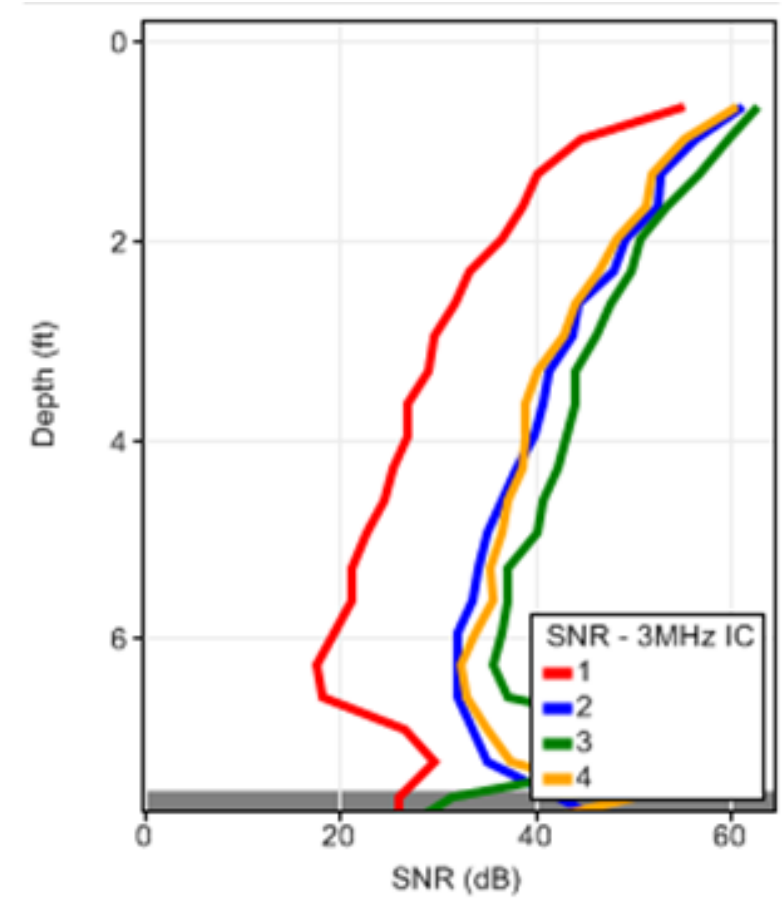

Figure 3. Example from RiverSurveyor Live of air entrainment partially blocking the acoustic signal in beam 1. [ft, feet; SNR, signal to noise ratio; dB, decibels; MHz, megahertz; IC, incoherent]. 


\section{Interpolation}

QRev interpolates water velocity data that have been determined to be invalid by using a different approach than that used by the manufacturers. Interpolation algorithms in RiverSurveyor Live are not available because filters for water data are not available. TRDI in WinRiver II does not interpolate water velocities but accounts for the invalid data by interpolating or extrapolating the cross product of the water and boat velocities used in the discharge computation (see "Computing Discharge from Invalid Data" section). QRev uses different approaches for estimating water velocities depending on whether all the depth cells in an ensemble have invalid water velocities or only some of depth cells have invalid water velocities.

If all the depth cells in an ensemble have invalid water velocities, QRev uses a twodimensional linear interpolation method called scatteredInterpolant, which is available in Matlab version $2015 \mathrm{~b}$. The $\mathrm{z}$ or normalized range from the streambed to the center of each depth cell and the distance made good along the ship track are used as the independent variables. Separate interpolations are completed for the $u$ and $v$ (east and north) velocity components. The interpolation method is set to linear with no extrapolation allowed.

If only some of the depth cells in an ensemble have invalid water velocities, the methods used in WinRiver II are applied to the cross product (Teledyne RD Instruments, 2014). However, QRev applies these methods to the individual velocity components. The method depends on the location of the depth cells with invalid water velocities and on the extrapolation method selected. If the depth cells with invalid water velocities are the topmost cells, the size of those depth cells is added to the top extrapolation distance and included in the top discharge. If the depth cells with invalid water velocities are the bottom most cells, the size of those depth cells is added to the bottom extrapolation distance and included in the bottom discharge. If depth cells with valid water velocities are above and below the depth cell(s) with invalid water velocities, the velocities of the invalid depth cells are interpolated. For these interior depth cells, the type of interpolation depends on the selected bottom extrapolation method. If the bottom extrapolation method is a power fit, the velocities for the invalid depth cells are estimated from the power fit. If the bottom extrapolation method is no slip, the velocities for the invalid depth cells are linearly interpolated from the valid depth cells immediately above and below the invalid cell. Although the application of the power fit to a velocity component that is not aligned with the current direction may appear to be a violation of the assumptions, the final computation of discharge is identical whether the interpolation is applied to the cross product or to the components from which the cross product is computed.

\section{Extrapolation Methods}

The ADCP is unable to measure the water column (fig. 4). Near the water surface, an unmeasured zone is associated with the immersion of the ADCP into the water and a distance below the transducers where valid data cannot be obtained because of ringing, flow disturbance, and ping configuration. The ADCP also cannot measure all the way to the streambed because of the potential for side lobe interference. The discharge estimates for the top and bottom unmeasured portions of the transect are dependent on the selected extrapolation methods. The extrapolation methods available in WinRiver II, RiverSurveyor Live, and QRev are similar.

- Top and bottom power fit - The power law (Chen, 1989) is applied to the data and used to extrapolate the top and bottom unmeasured areas. 
- Top constant-Assumes the velocity or discharge is constant from the uppermost depth cell to the water surface.

- Top three point - Uses a linear least squares extrapolation through the uppermost three depth cells to the water surface.

- Bottom no slip - Uses the assumption that the water velocity (and hence the discharge) should be zero at the solid boundary and fits a power curve through zero at the bottom and through depth cells in the lower 20 percent of the flow or the last valid depth cell, if no valid bins are in the bottom 20 percent of the water column (specific details may differ among manufacturers).

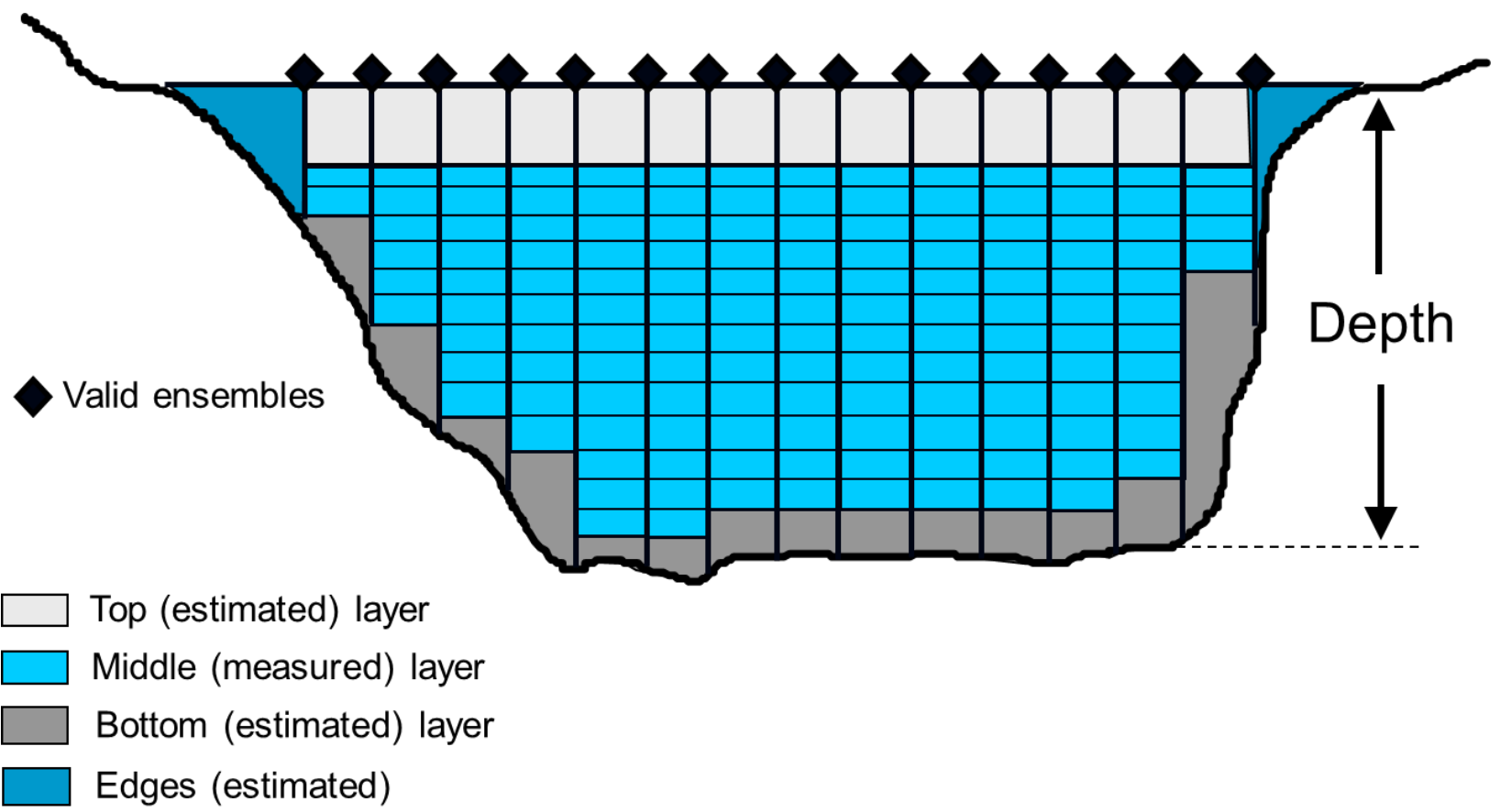

Figure 4. Illustration of measured and unmeasured zones of an acoustic Doppler current profiler transect.

The user is responsible for evaluating the profile and selecting the appropriate method for the top and bottom extrapolations. Currently (2015), the manufacturers' software assumes the manually selected extrapolation methods are valid for the transect. In other words, the extrapolation methods cannot be changed for different parts of the cross section.

Using the approach documented in Mueller (2013), QRev provides an automated selection of an appropriate extrapolation fit for the measurement. The automatically selected extrapolation method is the default method in QRev. However, the user should review the selected method to ensure a valid fit of the profile and make manual adjustments as appropriate.

The automatic approach uses linear least squares regression and several empirically developed criteria for selecting the appropriate extrapolation method. The automated method will attempt to select the best from among the following extrapolation methods:

- power fit through the profile with an exponent of 0.1667 (default),

- power fit through the profile with a linear least squares fit exponent,

- constant fit at the top and a no slip fit at the bottom with an exponent of 0.1667 , and 
- constant fit at the top and a no slip fit at the bottom with a linear least squares fit exponent.

The approach used in the automatic fit algorithm is that the data follow the power law with an exponent of 0.1667 unless the measured data are sufficient to prove otherwise. The following is a list of steps that are used to automatically select the appropriate extrapolation method.

1. Although the exponent for a power fit could be computed from a least squares fit of all of the data, visually assessing the appropriateness of the fit would be difficult and the fit could be influenced by outliers in the data. To provide a visual reference and to improve the method's robustness to outliers, the profile is subdivided into 5 percent increments of normalized depth. The mean normalized distance from streambed and median and interquartile range of normalized unit cross product for each increment are computed. The median of the unit cross products was selected to represent the mean profile, rather than the mean, because of the median's robustness to the influence of outliers. The median values of the unit cross product and their associated normalized distance from the streambed are used in the visual and computation approaches to determine the appropriate extrapolation methods.

2. The automatic method determines which of the 5 percent profile increments should be used in the analysis. Often the profile increments near the top and the bottom of the profile have substantially fewer data points contained in them. Data near the surface and particularly near the streambed are often noisier; therefore, with fewer data points in the medians, the medians of these increments may not be a good representation of the profile shape in these locations. The combination of these factors could adversely affect the evaluation of how well the proposed method fits the profile because all medians are given equal weight in the linear least squares regression. In addition, any median of a profile increment that does not contain more than 20 percent (default value, but user selectable) of the median number of points for all of the profile increments is marked invalid and is not used in the automatic analysis.

3. If the number of valid medians is six or less, the data are considered to be insufficient to reject the default assumption that the profile follows a power fit with an exponent of 0.1667 , and the power fit with an exponent of 0.1667 is automatically selected. These criteria were determined through analysis of many data sets by multiple persons experienced in the application of ADCPs for discharge measurements.

4. If seven or more valid medians exist, linear least squares regression is used to determine an optimized exponent for equation 22. An exponent of 0.1667 is assumed until the data prove a different exponent is appropriate. The 95 percent confidence intervals about the optimized exponent are used to determine if the data provide sufficient support to change the exponent. The optimized exponent is considered the appropriate exponent for the power fit of the profile if the computed coefficient of determination $\left(\mathrm{r}^{2}\right)$ from the regression is equal to or greater than 0.8 and the default exponent of 0.1667 is not contained within the 95 percent confidence intervals of the optimized exponent. If these conditions are not met, then the default 0.1667 exponent is retained.

5. Linear least squares regression is used to compute the exponent for the no slip fit. The bottom third of the medians are used in the regression rather than just 20 percent as is used for application of the no slip method in the discharge computations in the manufacturers' software. The additional data provided by using the bottom third of the 
profile help provide a smooth trend from the no slip extrapolation, with an optimized exponent determined from regression, to the measured data.

6. Using logic and specific criteria, developed empirically from review of many profiles, the best power fit is evaluated on how well it fits the top and bottom parts of the measured data. If the best power fit does not meet the prescribed criteria, a constant fit at the top and no slip fit at the bottom are selected.

7. If the $r^{2}$ from the linear regression in step 5 is greater than 0.8 , the no slip exponent computed from the regression is selected; if the $\mathrm{r}^{2}$ is not greater than 0.8 , the no slip exponent defaults to 0.1667 .

The automatic fit algorithms in QRev will select a limited combination of the available methods. If the top is not represented by a power fit, the top is set to constant and the bottom is set to no slip. The automatic fit algorithms will not select a constant fit for the top and power for the bottom fit. This combination, which has been used frequently in the past, creates a discontinuity at the top of the profile. If the profile does not follow a power fit, the bottom of the profile is better represented by the no slip fit. Likewise, the automatic fit algorithms will not select a threepoint fit for the top. A three-point fit may be appropriate to some situations and the user can manually select the three-point fit; however, the automatic algorithms do not have the logic to automatically select a three-point fit for the top.

\section{Discharge Computation}

The discharge computed by an ADCP is a summation of the measured portion of the cross section and extrapolated discharge estimates for unmeasured portions of the cross section at the top, bottom, and both edges (fig. 4). The discharge for the measured, top, and bottom portions of the cross section is computed for each ensemble, and the discharge computed for the edges is added to the total.

$$
Q=Q_{\text {LeftEdge }}+Q_{\text {Top }}+Q_{\text {Measured }}+Q_{\text {Bottom }}+Q_{\text {RightEdge }}
$$

where
$Q \quad$ is the total discharge,
$Q_{\text {LeftEdge }} \quad$ is the discharge estimated for the unmeasured area near the left bank,
$Q_{\text {Top }} \quad$ is the discharge estimated for the top unmeasured area,
$Q_{\text {Measured }}$ is the discharge measured directly by the ADCP,
$Q_{\text {Bottom }} \quad$ is the discharge estimated for the bottom unmeasured area, and
$Q_{\text {RightEdge }}$ is the discharge estimated for the unmeasured area near the right bank.

The general equations used to compute the measured and unmeasured portions of the cross section are well documented (Simpson and Oltmann, 1993; Mueller and others, 2013; Teledyne RD Instruments, 2014; SonTek, 2003) and are mostly consistent among the manufacturers; therefore, only a brief summary is provided herein. 


\section{Measured Discharge}

The measured discharge is computed using the cross product of the water and boat velocities. The equation for discharge in each depth cell, $Q_{b i n}$, can be written in terms of the water- and boat-velocity vector components as follows:

$$
Q_{b i n}=\left(\vec{V}_{w} \times \vec{V}_{b}\right) d t d z=\left(V_{w x} V_{b y}-V_{w y} V_{b x}\right) d t d z
$$

where

$Q_{\text {bin }} \quad$ is the discharge for a depth cell,

$d t \quad$ is the duration of the ensemble and

$d z \quad$ is the depth cell size.

The measured portion of the discharge can then be computed as follows:

$$
Q_{\text {Measured }}=\sum_{j=1}^{\text {Ensembles }} \sum_{i=1}^{\text {Bins }} Q_{\text {bin }}
$$

Because QRev interpolates invalid depth, boat velocity, and water velocities, all of the necessary data are available to apply equation 19.

\section{Top Discharge}

The top discharge is computed using the selected top extrapolation method-constant, power fit, or three-point fit. The extrapolation method for computing the top discharge can be applied to the individual velocity components (approach used by SonTek) or to the cross product from equation 19 (approach used by TRDI). Both approaches are mathematically identical. QRev uses the cross product; therefore, only the equations associated with that approach are presented herein.

\section{Constant}

The simplest assumption for estimating the top discharge is to assume that the velocity (cross product) in the topmost valid depth cell is a good estimate of the mean velocity between that depth cell and the water surface. This method is typically referred to as the constant extrapolation method as follows:

$$
Q_{\text {Top }}=\sum_{j=1}^{\text {Ensembles }} \chi\left(z_{w s}-z_{t b}\right) d t
$$

where

$$
\begin{aligned}
& \chi \\
& \begin{array}{l}
\text { is the velocity cross product in the topmost valid depth cell, } \\
z_{w s}
\end{array} \text { is the range from the streambed to the water surface, and } \\
& z_{t b} \quad \text { is the range from the streambed to the top of the topmost valid depth cell. }
\end{aligned}
$$

This constant extrapolation method is often used where an upstream wind or an irregular velocity profile exists through the measured portion of the water column. 


\section{Power}

The power fit is based on the power law (Chen, 1989). The power-law equation is represented in terms of the cross product as follows:

$$
\chi=a z^{b}
$$

where

$$
\begin{aligned}
& a \text { is a coefficient derived from a least-squares fit of the equation to the measured } \\
& \text { data, } \\
& z \quad \text { is the range from the streambed to the location of the value of } \chi \text {, and } \\
& b \quad \text { is the exponent commonly assumed to be } 1 / 6(0.1667) .
\end{aligned}
$$

Although 0.1667 is the default exponent for the power law, the exponent should be adjusted to fit the measured data as discussed in the "Extrapolation Methods" section of this report. The powerlaw equation is then integrated over the range from the water surface to the top of the uppermost depth cell with valid water velocities as follows:

$$
Q_{\text {Top }}=\sum_{j=1}^{\text {Ensembles }} \frac{a}{b+1}\left(z_{w s}^{b+1}-z_{t b}^{b+1}\right) d t
$$

Three-Point Fit

QRev uses the TRDI implementation of the three-point fit method for the top extrapolation. This method uses the top three bins to estimate a slope, and this slope is then applied from the top bin to the water surface. A constant value or slope of zero is assumed if less than six bins are present in the profile (Teledyne RD Instruments, 2014).

$$
Q_{\text {Top }}=\left(\frac{A d_{T}^{2}}{2}+B d_{T}\right) d t
$$

where

$$
\begin{gathered}
A=\frac{3 \sum_{i=1}^{3} \chi_{i} d_{i}-\sum_{i=1}^{3} \chi_{i} \sum_{i=1}^{3} d_{i}}{3 \sum_{i=1}^{3} d_{i}^{2}-\left(\sum_{i=1}^{3} d_{i}\right)^{2}} \\
B=\frac{\sum_{i=1}^{3} \chi_{i} \sum_{i=1}^{3} d_{i}^{2}-\sum_{i=1}^{3} \chi_{i} d_{i} \sum_{i=1}^{3} d_{i}}{3 \sum_{i=1}^{3} d_{i}^{2}-\left(\sum_{i=1}^{3} d_{i}\right)^{2}}
\end{gathered}
$$

$d_{T} \quad$ is the range from the water surface to the top of the topmost valid bin; and $d_{i} \quad$ is one of the top three valid bins. 


\section{Bottom Discharge}

ADCPs cannot measure the water velocity near the streambed because of side lobe interference. Unlike the top discharge estimation problem where the velocity at the water surface is not known, the water velocity at the streambed is somewhat known. Fluid mechanics theory indicates the water velocity must go to zero at the streambed and that a logarithmic velocity profile is a reasonable approximation of the velocity profile in the boundary layer (Schlichting, 1979). Therefore, the power law is always used to compute the discharge in the bottom unmeasured portion of the water column as follows:

$$
Q_{\text {Bottom }}=\sum_{j=1}^{\text {Ensembles }} \frac{a}{b+1} z_{b b}{ }^{b+1} d t
$$

where

$$
\begin{aligned}
& z_{b b} \quad \text { is the range from the streambed to the bottom of the bottom most valid depth } \\
& \text { cell. }
\end{aligned}
$$

To better apply this method to situations where the profile throughout the water column may not follow a logarithmic distribution, such as for bidirectional flow, a no slip method is used. The no slip method applies equation 27 but restricts the least-squares determination of $a$ to depth cells in the bottom 20 percent of the profile, or in the absence of valid depth cells in the bottom 20 percent, the last valid depth cell is used to compute $a$.

\section{Edge Discharge}

The unmeasured discharge at the edges of the stream are estimated using a ratio interpolation method documented by Fulford and Sauer (1986), which can be used to estimate a velocity at an unmeasured location between the riverbank and the first or last measured velocity in a cross section. The equation for the estimate is as follows:

$$
\frac{V_{x}}{\sqrt{d_{x}}}=\frac{V_{m}}{\sqrt{d_{m}}}
$$

where

\footnotetext{
$x \quad$ is a location midway between the riverbank and edge ensemble or L/2 (where $\mathrm{L}$ is measured and entered by the user),

$V_{x} \quad$ is the estimated mean velocity at location $x$,

$d_{x} \quad$ is the depth at location $x$,

$V_{m} \quad$ is the measured mean velocity at the first or last acoustic Doppler current profiler-measured subsection, and

$d_{m} \quad$ is the depth at the first or last acoustic Doppler current profiler-measured ensemble.
} 
Fulford and Sauer (1986) defined $d_{m}$ and $V_{m}$ as depth and velocity, respectively, at the center of the first or last measured subsection and not the near-shore edge of the ensemble, as presented in equation 28. However, because the ADCP ensembles purposely are kept narrow at the start and finish of each measurement, the differences between the two applications are not significant (Simpson and Oltmann, 1993). With the Fulford and Sauer (1986) method, discharge can be estimated by assuming a triangular area at the edge as follows:

$$
Q_{E d g e}=A_{\text {edge }} V_{L / 2}=0.5 L d_{m} * V_{m} \frac{\sqrt{0.5 d_{m}}}{\sqrt{d_{m}}}=0.3535 L d_{m} V_{m}
$$

where

$Q_{\text {Edge }}$ is the estimated discharge in the unmeasured edge,

$A_{\text {edge }}$ is the area of the unmeasured edge,

$V_{L / 2} \quad$ is the velocity midway between the bank and the first or last acoustic Doppler current profiler-measured ensemble, and

$L \quad$ is the distance from the last valid ensemble to the edge of water.

Equation 29 can be written in a more general form, which uses an edge-shape coefficient, as follows:

$$
Q_{E d g e}=C_{e} V_{m} L d_{m}
$$

where

$$
C_{e} \quad \text { is an edge-shape coefficient. }
$$

The edge-shape coefficient is a function of the shape, roughness, and velocity distribution in the unmeasured edge. For triangular edges, the coefficient is commonly set to 0.3535 . Oberg and Schmidt (1994) used 0.91 for rectangular edges, and 0.91 is the default value for rectangular edges used in WinRiver II (Teledyne RD Instruments, 2014). SonTek (2003) used data from Rantz and others (1982) to develop an equation for the rectangular edge coefficient based on the ratio of $L / d_{m}$ as follows:

$$
C_{e}=\frac{1-\frac{0.35}{4} \frac{d_{m}}{L}\left(1-e^{-4 \frac{L}{d_{m}}}\right)}{1-0.35 e^{-4 \frac{L}{d_{m}}}}
$$

Investigation into the development of equation 31 by SonTek resulted in a third alternative for the rectangular edge coefficient but also provided some information on sensitivity associated with computing the rectangular edge coefficient. The proposed rectangular edge coefficients refer to a set of laboratory data (table 5) that do not have a reliable reference. Rantz and others (1982) indicated that "Laboratory data suggest ..." and provide no reference. Khan and others (1997) present the same data and provide a reference to Hagan (1989). Hagan (1989) was not readily available. SonTek fit these data with the following equation: 


$$
\frac{V_{x}}{V_{D}}=1-0.35 e^{-4 \frac{x}{D}}
$$

where

$V_{x} \quad$ is the velocity measured some distance from the vertical wall,

$V_{D} \quad$ is the velocity at a distance from the vertical wall equal to the depth,

$x \quad$ is the distance from the wall, and

$D \quad$ is the depth at the wall.

Using the curve fit toolbox in Matlab, the following equation was fit to these same data:

$$
\frac{V_{x}}{V_{D}}=0.9097 e^{0.0947 \frac{x}{D}}-0.2597 e^{-8.4365 \frac{x}{D}}
$$

The SonTek and Matlab fit are compared to the data presented in Rantz and others (1982), and a distinct difference between the SonTek and Matlab fits is shown in figure 5. SonTek's fit attempts to maintain a value of $\frac{V_{x}}{V_{D}}$ near 1 for values of $\frac{x}{D}$ greater than or equal to 1 . Because the available data do not extend beyond $\frac{x}{D}=1$, the proper behavior is unknown. However, the velocity of streams generally increase from the fixed boundary towards the center of the channel (Seo and Baek, 2004), although the shape of the velocity distribution across the channel will vary with channel geometry and roughness. Integrating equation 33 and manipulation of the equation to compute the edge discharge results in a rectangular edge coefficient defined by equation 34 as follows:

$$
C_{e}=\frac{\left(\frac{d_{m}}{L}\right)\left(9.606 e^{0.0947 \frac{L}{d_{m}}}+0.0308 e^{-8.433 \frac{L}{d_{m}}}-9.637\right)}{0.910 e^{0.0947 \frac{L}{d_{m}}}-0.260 e^{-8.436 \frac{L}{d_{m}}}}
$$

The rectangular edge coefficient options for values of $\frac{L}{d_{m}}$ from 0 to 2 are compared in figure 6. Because the velocity of streams generally increase from the fixed boundary towards the center of the channel as previously noted, the edge coefficient would seem to decrease as $\frac{L}{d_{m}}$ increases. The edge coefficient computed from the SonTek equation increases rather than decreases. Given that the range of variation in the edge coefficient is about $+/-0.02$ and the disagreement in the behavior of the two equations presented, QRev will default to 0.91 until additional research is completed and a more accurate approach is documented. 
Table 5. Laboratory data on velocity near vertical walls (Rantz and others, 1982).

\begin{tabular}{|l|l|}
\hline $\begin{array}{l}\text { Distance from wall, as a ratio } \\
\text { of depth }\end{array}$ & $\begin{array}{l}\text { Mean vertical velocity, as related to the velocity }\left(V_{D}\right) \text { at a } \\
\text { distance of one depth from the wall }\end{array}$ \\
\hline 0.00 & $0.65 V_{D}$ \\
\hline 0.25 & $0.90 V_{D}$ \\
\hline 0.50 & $0.95 V_{D}$ \\
\hline 1.00 & $1.00 V_{D}$ \\
\hline
\end{tabular}

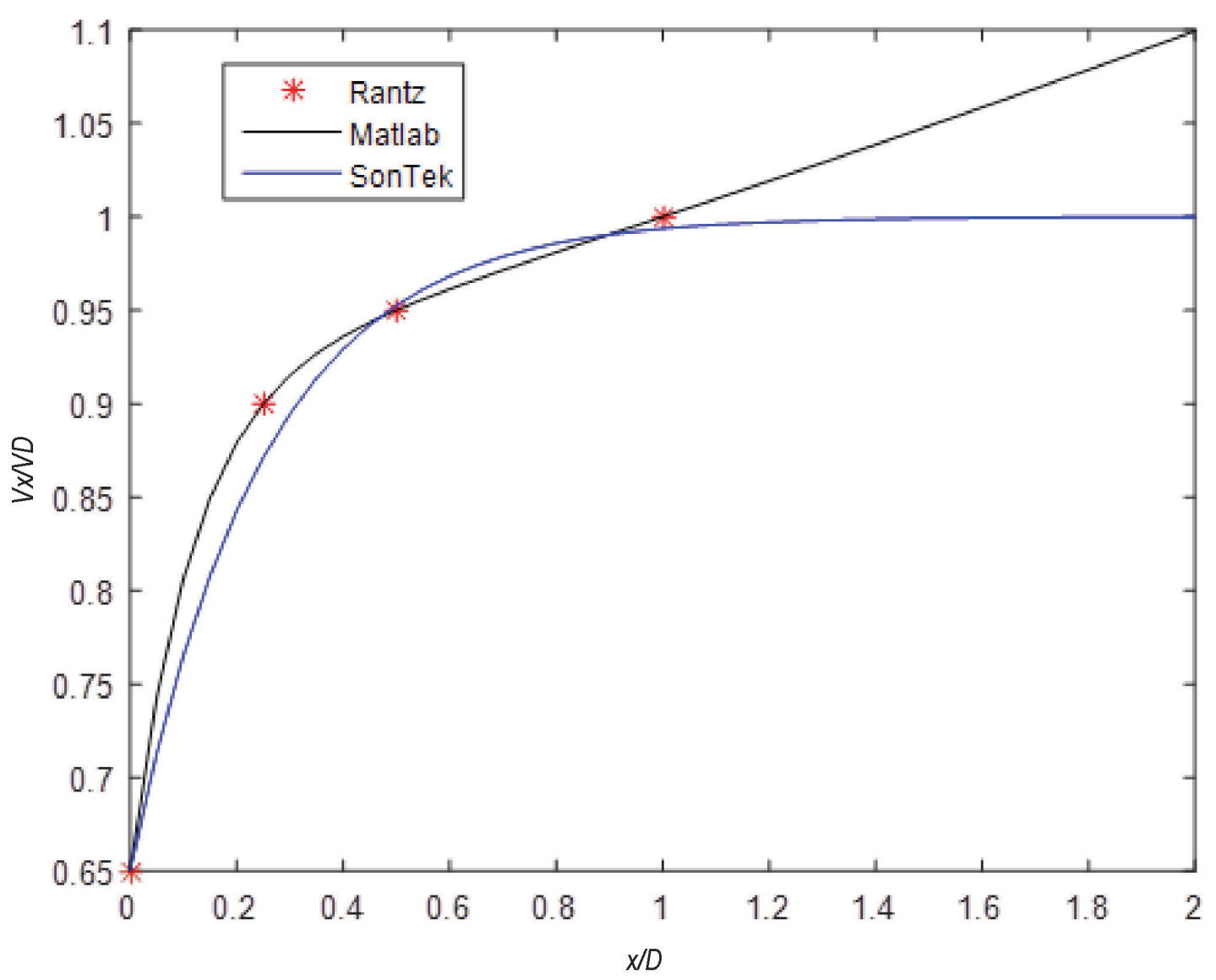

Figure 5. Comparison of fits to data from Rantz and others (1982). [ $V x$, velocity measured some distance from the vertical wall; $V D$, velocity at a distance from the vertical wall equal to the depth; $x$, distance from the wall; $D$, depth at the wall.] 


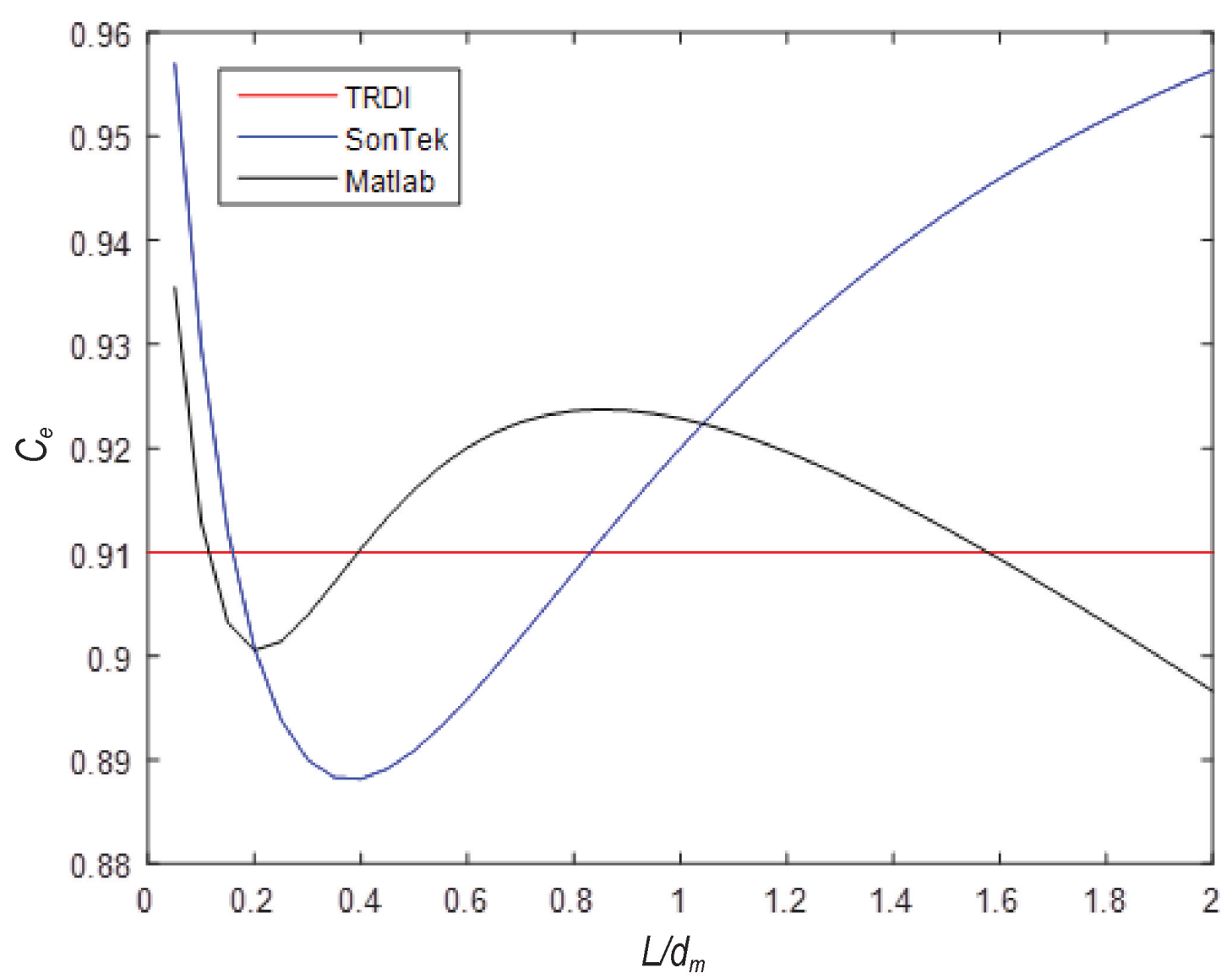

Figure 6. Comparison of proposed rectangular edge coefficients. [ $C_{e}$, edge coefficient; $L$, distance from the wall; $d_{\mathrm{m}}$, depth at the wall]

The value for $L$ must be measured and entered by the user. To obtain a good measurement for $V_{m}$, at least 10 ensembles of valid data also are recommended to be collected in a near-stationary position at the beginning and end of each transect. QRev follows WinRiver II procedures for data collected with WinRiver II; the first or last 10 valid ensembles are used. For data collected with RiverSurveyor Live, QRev honors the RiverSurveyor Live designation of edge ensembles and if none of the edge ensembles are valid, a zero edge discharge is computed. The user is presented a warning in such cases and can manually adjust the number of edge ensembles.

\section{Discharge for Invalid Data}

The discharge for ensembles with invalid data is computed from the interpolated values of boat velocity, depth, and water velocity. By interpolating these basic variables, the discharge is computed rather than interpolated. Boat velocity, depth, and water velocity are never extrapolated; therefore, ensembles at the beginning or end of a transect will have a zero discharge if the boat velocity, depth, or water velocity data are invalid. 


\section{Measurement Quality Assessment}

The person making the discharge measurement is concerned with the quality of the data being collected. QRev assists the user by automatically checking many quality indicators and providing color coded and textual feedback on the results of the automated quality checks. Some of the checks enforce USGS policy associated with using ADCP's for moving-boat streamflow measurements, and other checks help identify potential issues with the collected data.

\section{Transects}

The USGS policy is that a measurement be comprised of at least one pair of reciprocal transects (two transects) and that the combined duration of all transects be at least 720 seconds (Mueller and others, 2013). A single transect may be considered a measurement in situations with rapidly varying flow or for some tidal measurements. QRev checks for consistency with USGS policy and provides guidance where the combination of transects may not be optimal. Typically, all transects in a measurement should have the same sign (positive or negative), except in rare circumstances. Generally, a change in sign is due to user error in assigning the correct start bank. The uncertainty of measurements with only two transects can often be reduced by collecting additional transects. QRev checks for these situations and provides the user feedback as shown in figure 7.

\section{System Test}

The USGS policy is that a system test be completed prior to making a discharge measurement with an ADCP (Mueller and others, 2013). System test results for TRDI ADCPs are typically stored in the *.mmt file and for SonTek ADCPs are stored in the SystemTest folder. If the test results are in these locations, QRev will automatically load the results. However, if results of a system test are stored elsewhere, the user can manually load the file. Results from multiple system tests can be loaded. The results will be identified by date and time, or if loaded manually, the results will be identified by filename.

A system test is actually a series of different tests for various aspects of the ADCP. A complete series of discrete tests is referred to as a "system test", and a single test within that series is referred to as a "discrete test". QRev automatically scans the results, reports the number of failed tests, and provides feedback to the user by coloring the system test button and providing messages as shown in figure 8 .

\section{Compass Calibration and Evaluation}

Most ADCPs contain an internal compass and procedures for calibrating the compass. In addition, some ADCPs have procedures for evaluating the compass after an initial calibration. A compass calibration is required if a loop moving-bed test is used or if the boat velocity is referenced to GGA or VTG. If the boat velocity is referenced to bottom track and a stationary moving-bed test is used, the compass will have no effect on the resulting discharge. However, a compass calibration is still encouraged so that the direction of the velocity data will be correct for any use of the data other than discharge. A calibration and evaluation should be completed for TRDI ADCPs and are required in QRev to receive a "good" assessment. SonTek ADCPs do not have an evaluation function so a "good" assessment is assigned if the compass was calibrated. The logic for assessing the compass calibration and evaluation in QRev is provided in figure 9. QRev only determines if a calibration or evaluation, or both have been completed. 
Assessing the quality of the calibration or evaluation, or both will be considered for future versions of QRev.

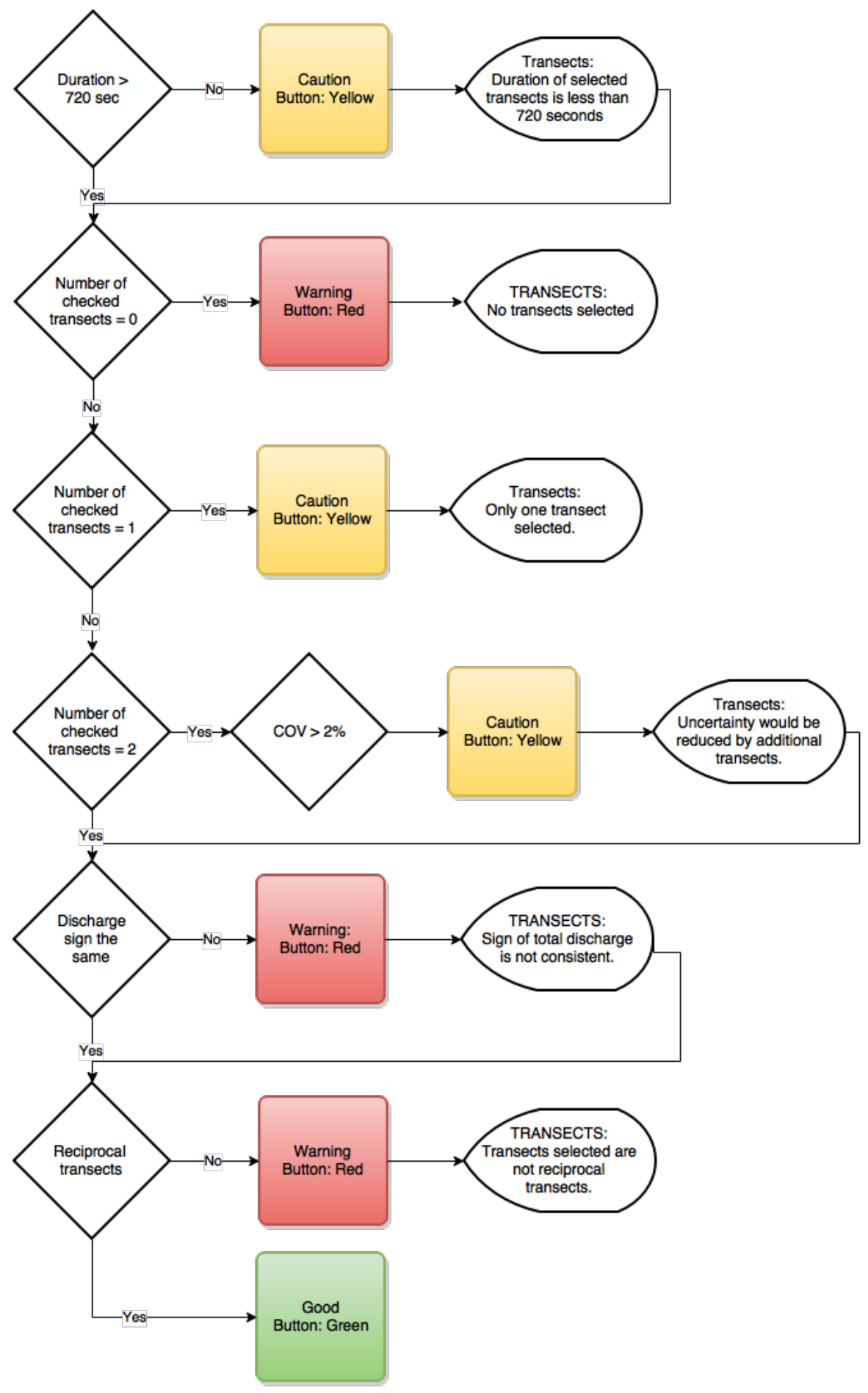

Figure 7. Flowchart of transects assessment. [COV, coefficient of variation] 


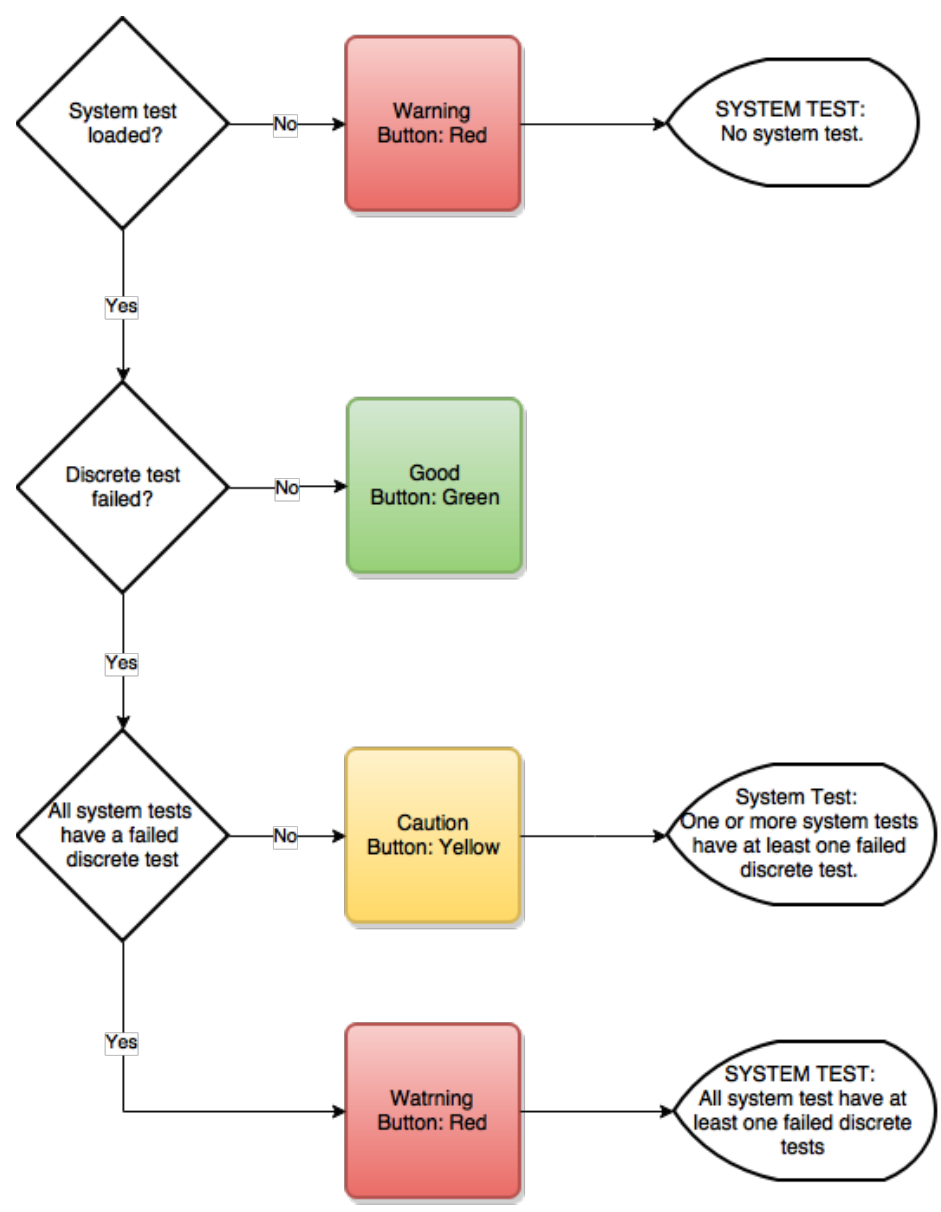

Figure 8. Flowchart of system test assessment. 


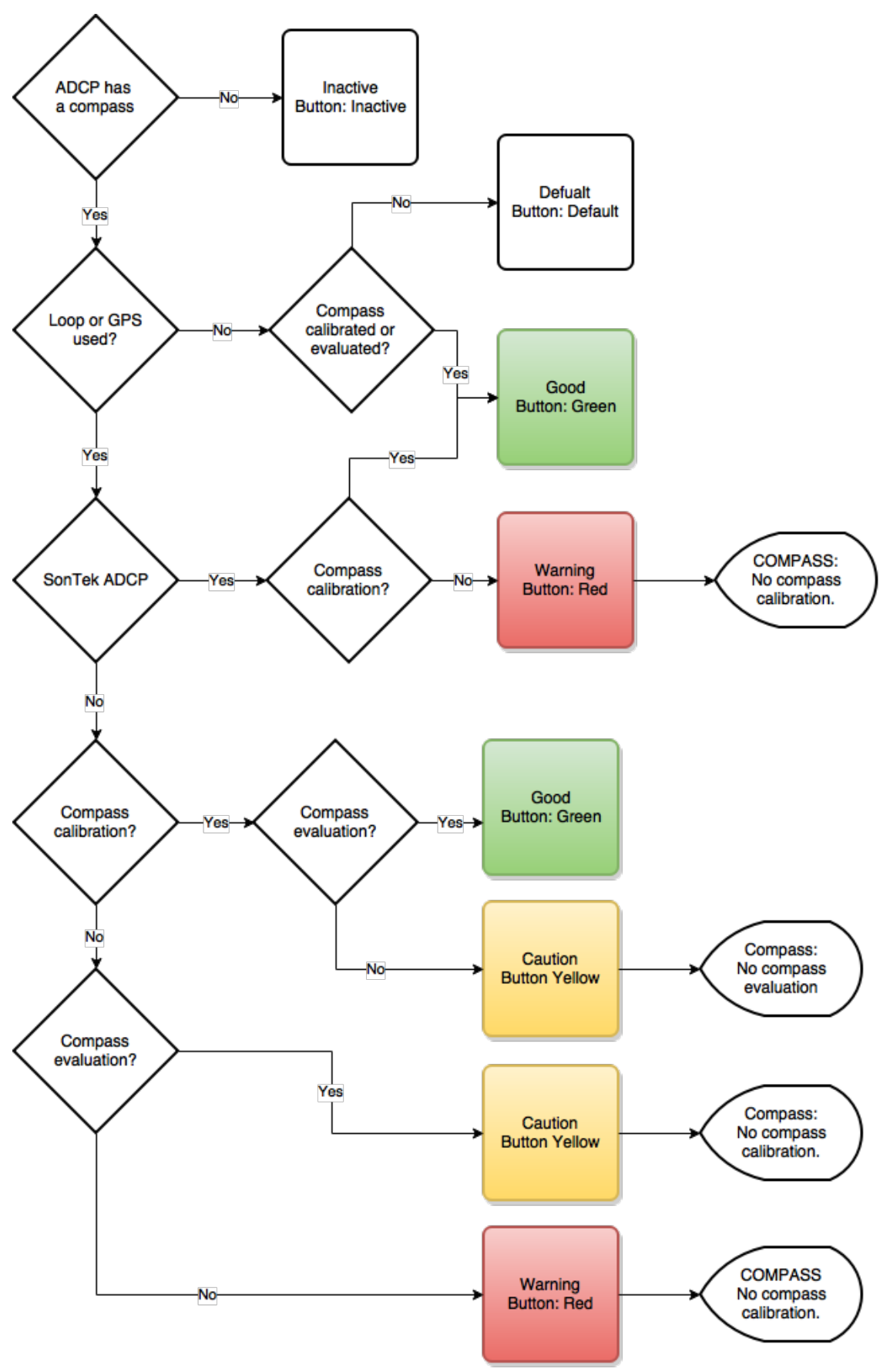

Figure 9. Flowchart of compass calibration and evaluation quality assessment. [GPS, global positioning system; ADCP, acoustic Doppler current profiler] 


\section{Water Temperature Validation}

The accuracy of discharge measurements made with an ADCP is dependent on an accurate speed of sound, which is dependent on an accurate water temperature. The USGS policy is that an independent water temperature be collected and compared to the ADCP's reported water temperature for each measurement. WinRiver II allows the user to enter this independent water temperature, and QRev will read that value from the *.mmt file and automatically populate that value in QRev. When using SonTek ADCPs with QRev, the user must manually enter the independent water temperature in QRev. QRev compares the independent water temperature to a user entered ADCP reading or the mean ADCP water temperature for the whole measurement. Consistent with USGS policy, if the difference in temperatures exceed 2 degrees Celsius, a warning is issued.

QRev also evaluates the change in ADCP measured water temperature for the duration of the measurement. At some sites, the water temperature may change during the measurement or spatially in the cross section. However, a change in measured water temperature during a measurement is often indicative of the ADCP not being given sufficient time to equilibrate to the water temperature. In this situation, the ADCP is actually measuring the wrong water temperature and, thus, using the wrong speed of sound. The logic used to assess water temperature is provided in figure 10 .

\section{Moving-Bed Tests}

The USGS policy requires a moving-bed test to be completed for every ADCP movingboat discharge measurement (Mueller and others, 2013). A loop or stationary moving-bed test method can be used. The quality assessment of moving-bed tests is done in the following three steps: (1) detailed evaluation of each moving-bed test, (2) selection of the moving-bed test(s) to be used, and (3) general quality assessment using the selected test(s). The detailed evaluation step evaluates the actual moving-bed test based on specific criteria for loop or stationary tests. Based on the results of that detailed evaluation, QRev selects the moving-bed test(s) to use to determine if a moving bed exists and applies any necessary corrections. The general quality assessment step is completed on the results of the selected moving-bed test and provides the color coded and textual feedback to the user in the main QRev window.

The evaluation for loop tests uses the same criteria and algorithms used in the computer program LC (Mueller and others, 2013) and gives the loop moving-bed test a quality rating of good, warnings, or errors. The specific messages related to each loop moving-bed test are displayed in the moving-bed window and not in the main window. The quality of the loop data are evaluated and given a rating of good, warnings, or errors (fig. 11). If an error exists, the loop test is not valid and the moving-bed condition remains unknown. If the loop test is valid, the results of the loop test are evaluated to determine if a moving-bed condition exists or not (fig. 12).

Unlike WinRiver II, RiverSurveyor Live, or SMBA, QRev evaluates the quality of individual stationary moving-bed tests. The evaluation examines the percentage of ensembles with invalid bottom track velocities, the duration of the test, and if the test appears to have reached equilibrium. The logic for the evaluation of stationary moving-bed test is provided in figure 13 . 




Figure 10. Flowchart of temperature assessment. [ADCP, acoustic Doppler current profiler] 

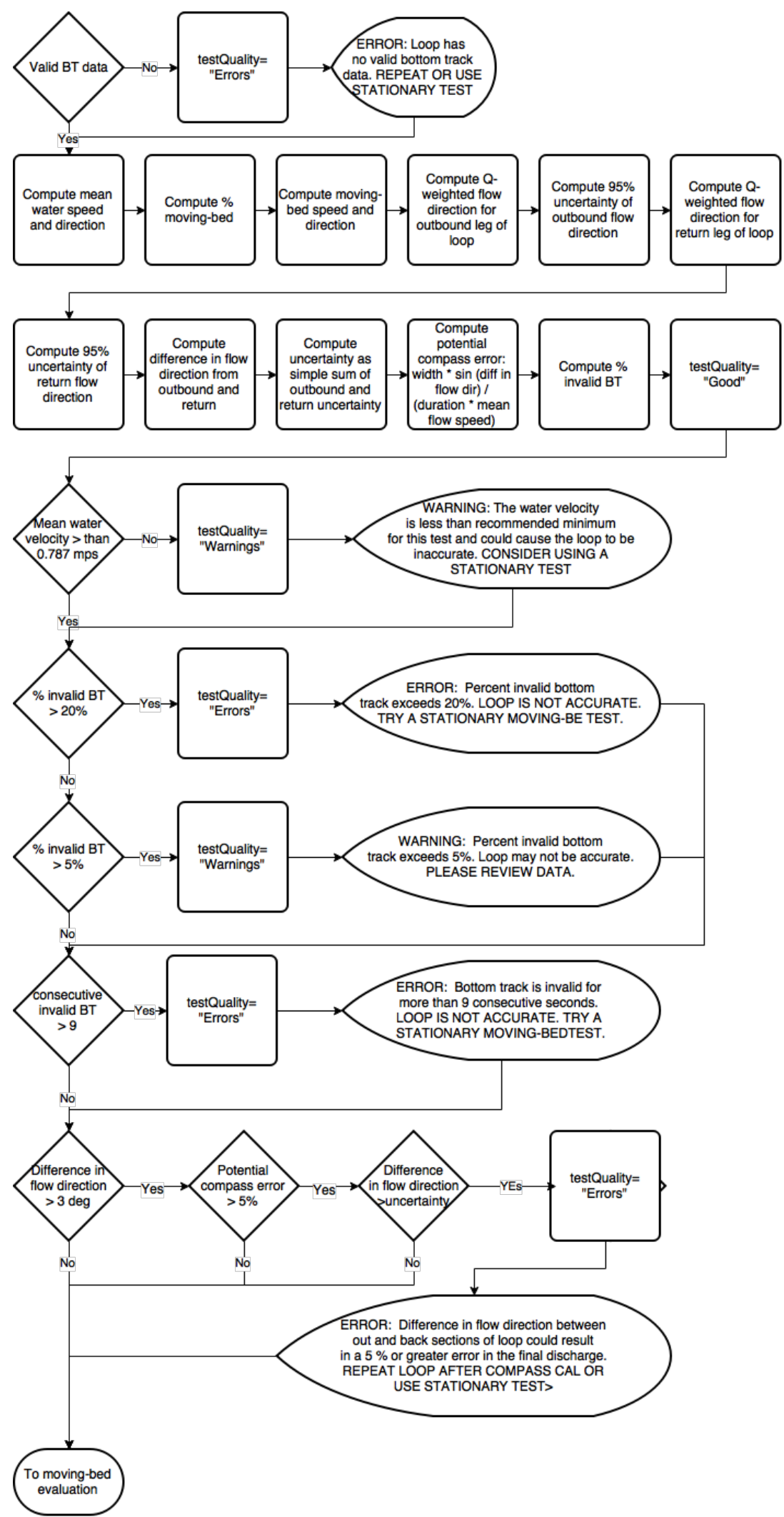

Figure 11. Flowchart of loop moving-bed test data quality evaluation. [BT, bottom track] 


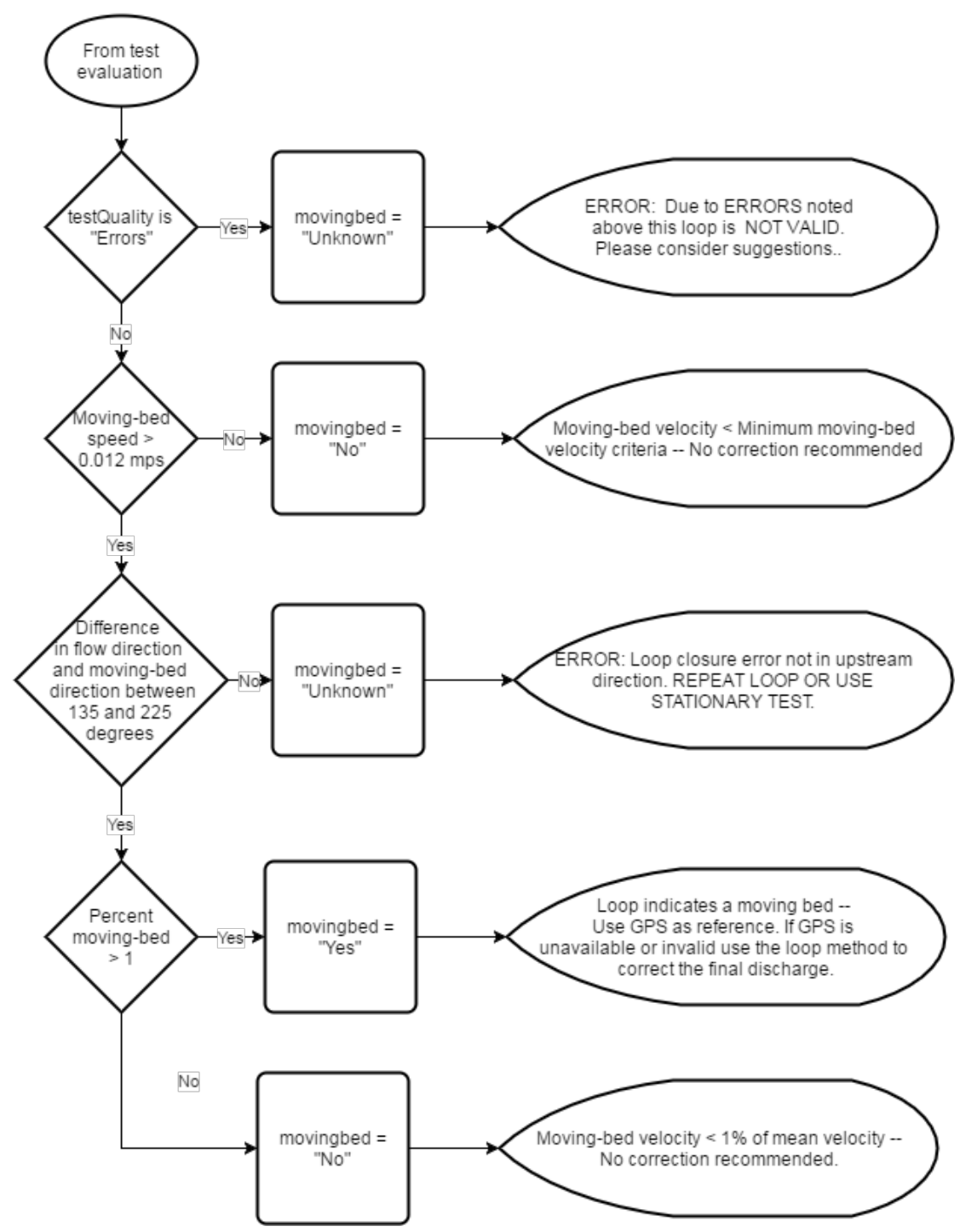

Figure 12. Flowchart of loop moving-bed test moving-bed evaluation. 


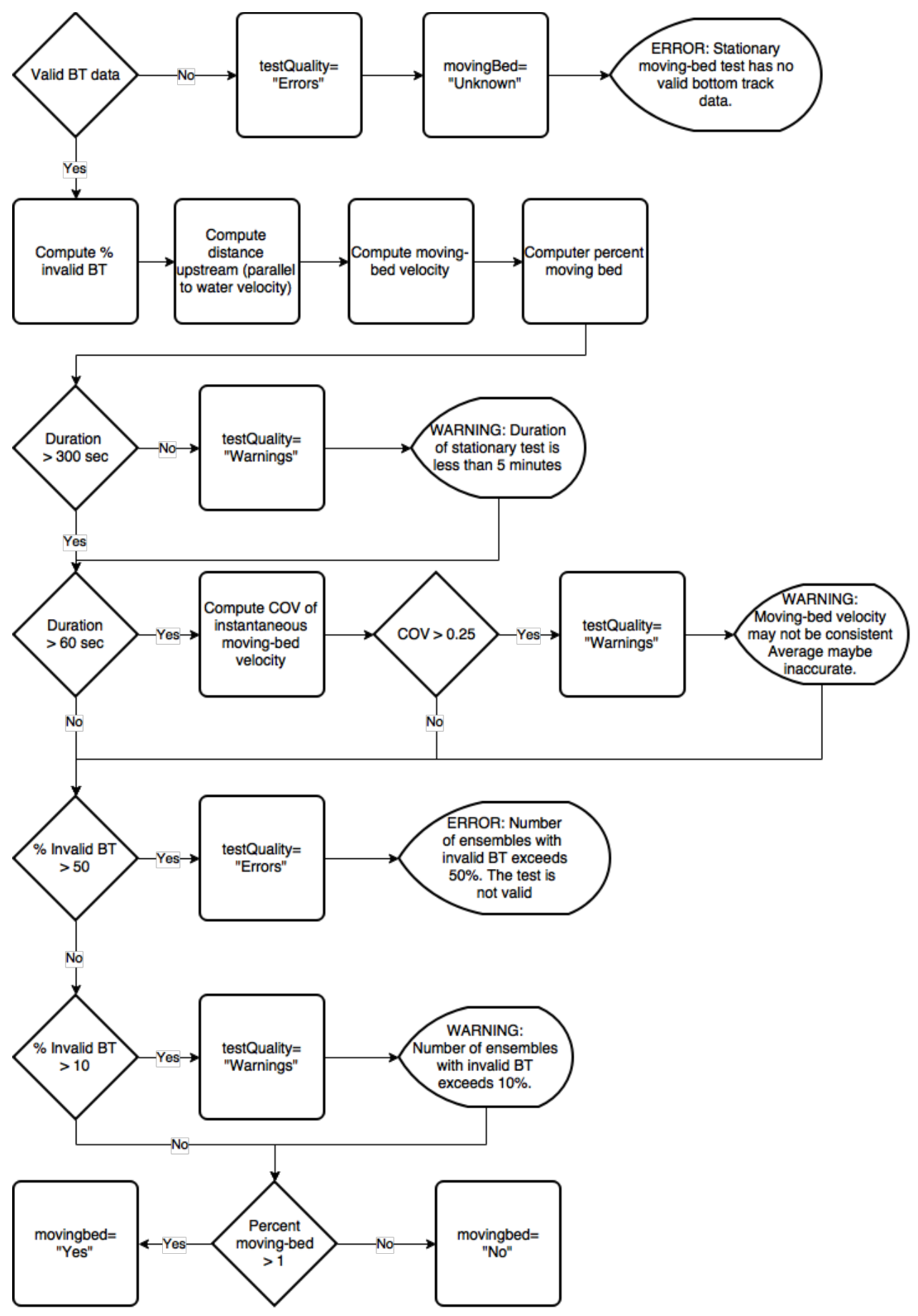

Figure 13. Flowchart of stationary moving-bed test evaluation. [BT, bottom track; COV, coefficient of variation] 
If more than one loop test or a loop test(s) and stationary test(s) are completed, QRev must decide which test(s) should be selected to determine if a moving bed exists and compute any required moving-bed corrections. If more than one valid loop test exits, the last valid loop test is selected. If no valid loop tests exist, all valid stationary tests are selected. If any of the selected tests indicate a moving bed, a moving-bed condition exists and the selected test(s) is used to correct the bottom track referenced discharge. The logic for selecting the moving-bed test is provided in figure 14 .

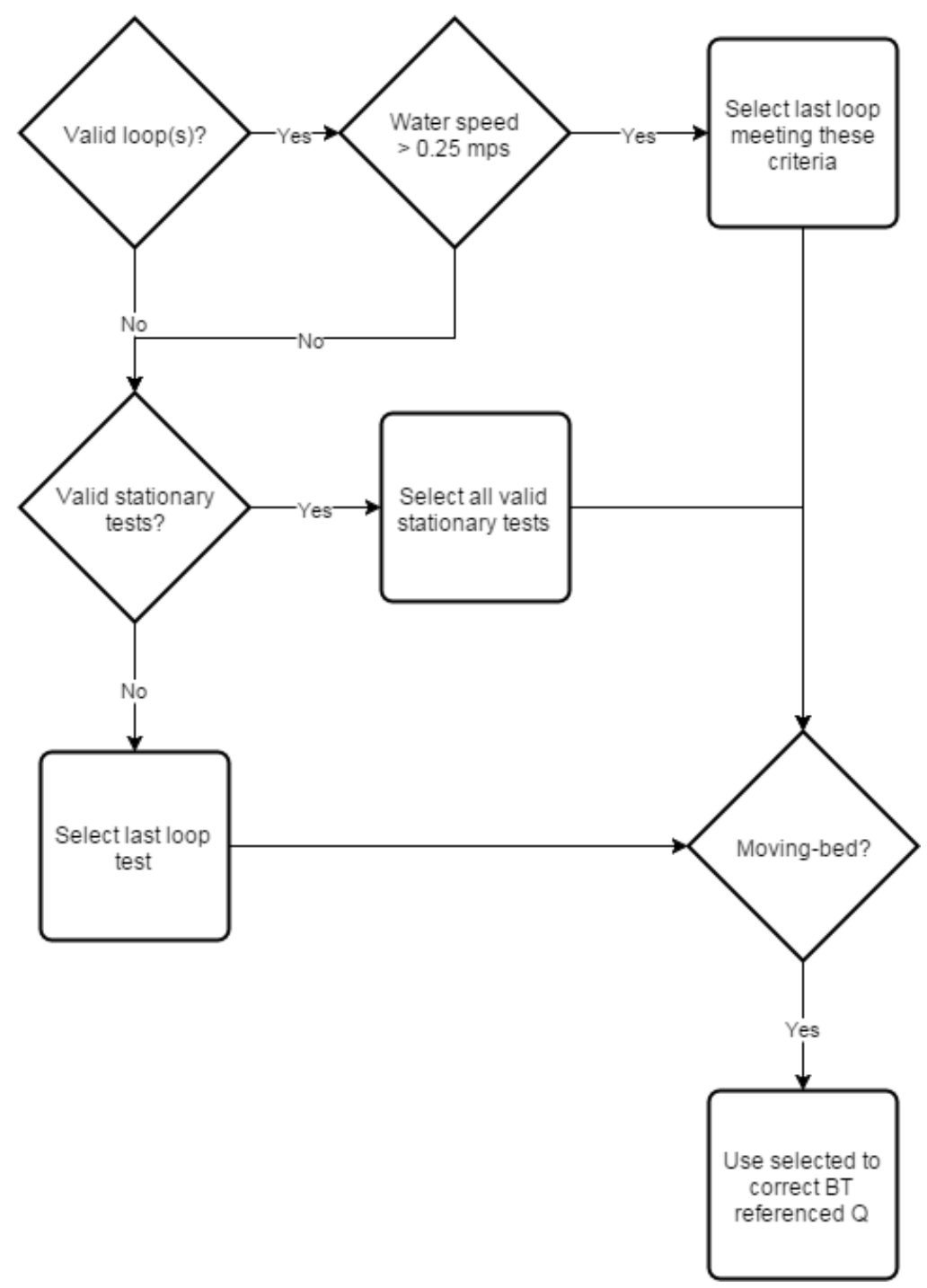

Figure 14. Flowchart for selecting from multiple moving-bed tests. [mps, meters per second] 
The general quality assessment uses the selected moving-bed test to set the button color and provide messages to the user. The logic for the general assessment step of moving-bed tests is provided in figure 15 .

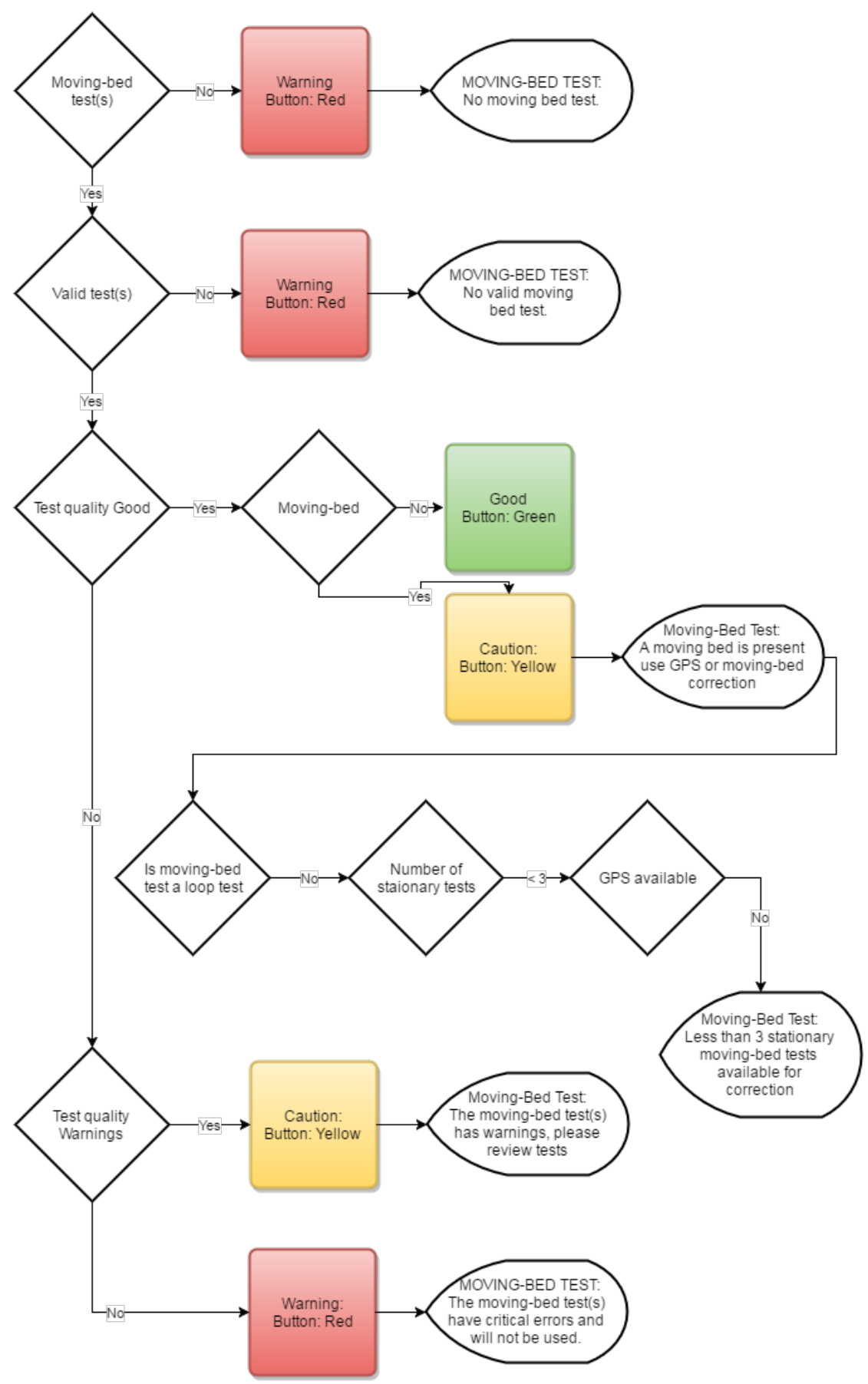

Figure 15. Flowchart of moving-bed test assessment. [GPS, global positioning system] 


\section{User Input}

User input for the ADCP draft and magnetic variation is evaluated for reasonableness and consistency. In addition, QRev checks to see that a station name and station number have been entered. If the station name and number are entered in WinRiver II, QRev will read and use those values. The Matlab output from SonTek does not provide a station name and number; therefore, for SonTek ADCPs, the user must enter the station name and number manually in QRev. The logic for assessing the user input is provided in figure 16.

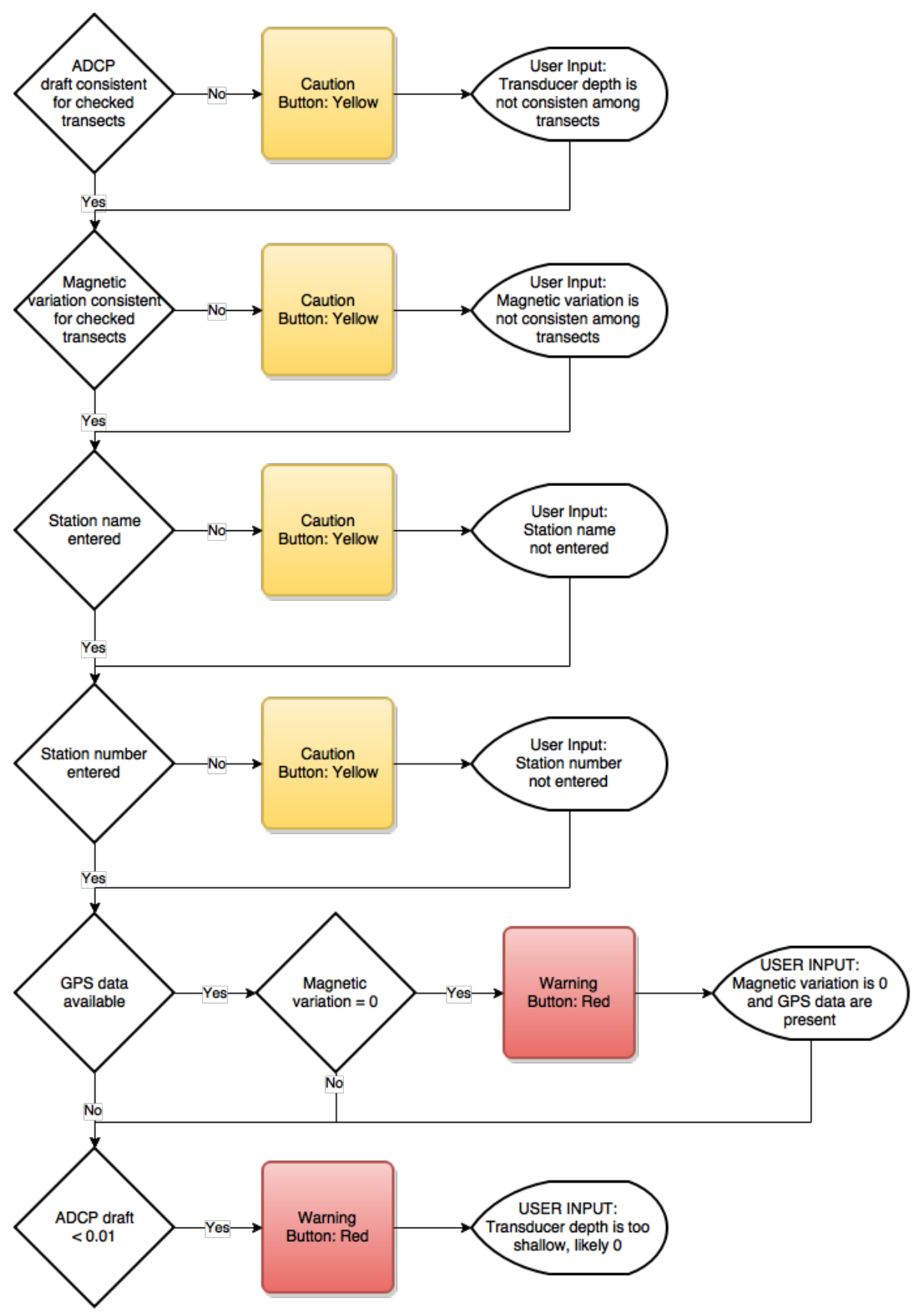

Figure 16. Flowchart of user input assessment. [ADCP, acoustic Doppler current profiler] 


\section{Measured Discharge Variables}

The measured discharge variables are boat velocity, depth, and water velocity. For each of these variables, the ensembles with invalid data are identified and the discharge is computed for those invalid ensembles based on the interpolated data determined. The quality assessment is based on the following three characteristics: (1) the total discharge interpolated, (2) the maximum discharge interpolated for a continuous segment of invalid data, and (3) the total number of invalid ensembles. The logic and threshold used to provide feedback are provided in figure 17 . The messages to the user resulting from the assessment are listed in tables 6-8.

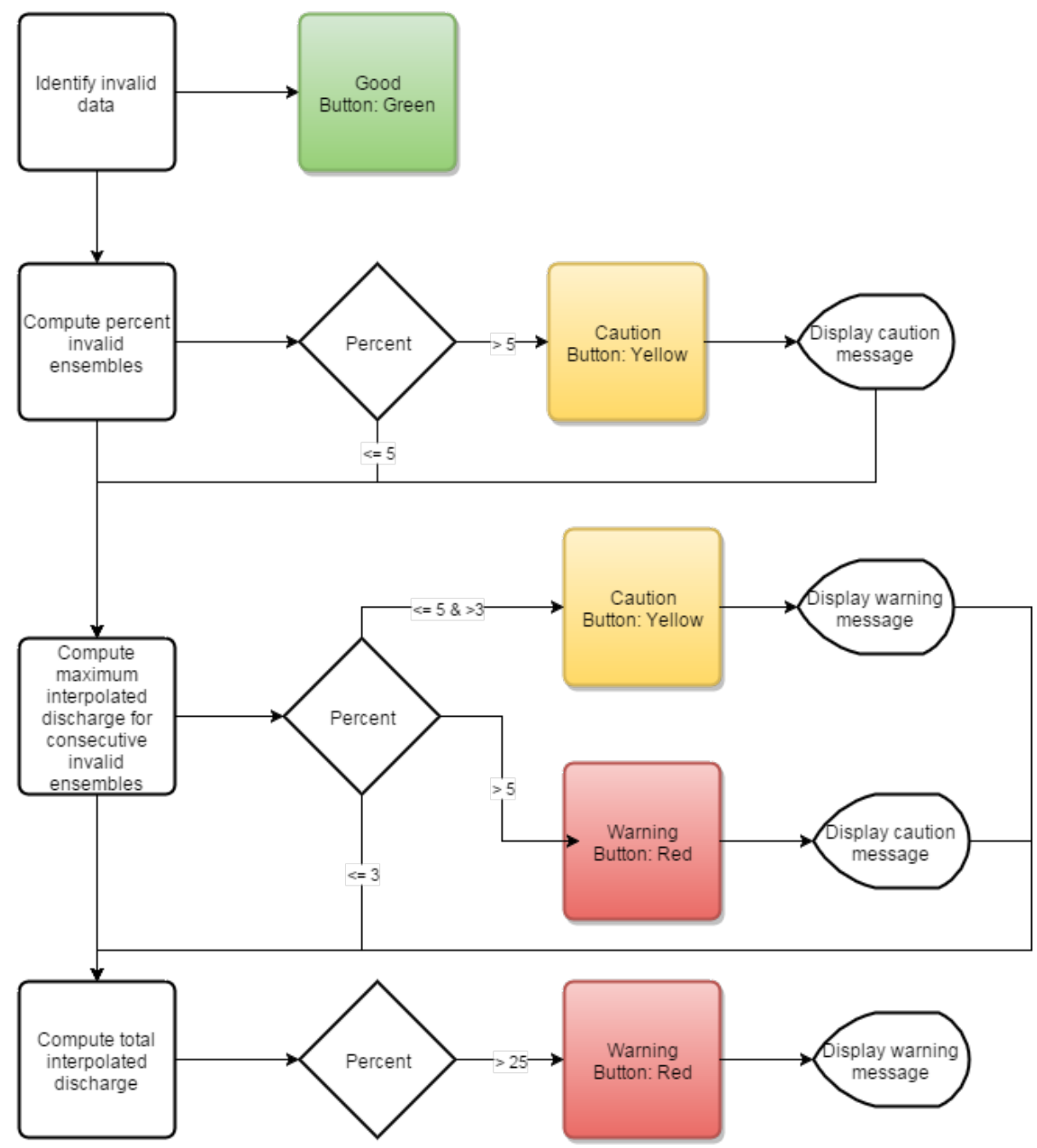

Figure 17. Flowchart of measured discharge variable (boat velocity, depth, and water velocity) assessment. 
Table 6. Summary of quality assessment messages for depth.

\begin{tabular}{|l|l|}
\hline Type & Message \\
\hline Caution & Depth: The percentage of invalid ensembles in a transect exceeds 5\%. \\
\hline Caution & Depth: Interpolated discharge for consecutive invalid ensembles exceeds $3 \%$. \\
\hline Warning & DEPTH: Interpolated discharge for consecutive invalid ensembles exceeds $5 \%$. \\
\hline Warning & DEPTH: Interpolated discharge for invalid ensembles in a transect exceeds $25 \%$. \\
\hline
\end{tabular}

Table 7. Summary of quality assessment messages for boat velocity.

\begin{tabular}{|c|c|c|c|}
\hline Type & Reference & Filter & Message \\
\hline \multirow[t]{2}{*}{ Caution } & bt- & $\begin{array}{l}\text { All: } \\
\text { Original: } \\
\text { ErrorVel: } \\
\text { VertVel: } \\
\text { Other: } \\
\text { 3Beams: }\end{array}$ & \multirow[t]{2}{*}{ The percentage of invalid ensembles in a transect exceeds $5 \%$. } \\
\hline & $\begin{array}{l}\text { gga- } \\
\text { vtg- }\end{array}$ & $\begin{array}{l}\text { All: } \\
\text { Original: } \\
\text { DGPS: } \\
\text { Altitude: } \\
\text { Other: } \\
\text { HDOP: }\end{array}$ & \\
\hline \multirow[t]{2}{*}{ Caution } & bt- & $\begin{array}{l}\text { All: } \\
\text { Original: } \\
\text { ErrorVel: } \\
\text { VertVel: } \\
\text { Other: } \\
\text { 3Beams: }\end{array}$ & \multirow[t]{2}{*}{ Interpolated discharge for consecutive invalid ensembles exceeds $3 \%$. } \\
\hline & $\begin{array}{l}\text { gga- } \\
\text { vtg- }\end{array}$ & $\begin{array}{l}\text { All: } \\
\text { Original: } \\
\text { DGPS: } \\
\text { Altitude: } \\
\text { Other: } \\
\text { HDOP: }\end{array}$ & \\
\hline \multirow[t]{2}{*}{ Warning } & BT- & $\begin{array}{l}\text { All: } \\
\text { Original: } \\
\text { ErrorVel: } \\
\text { VertVel: } \\
\text { Other: } \\
\text { 3Beams: }\end{array}$ & \multirow[t]{2}{*}{ Interpolated discharge for consecutive invalid ensembles exceeds $5 \%$. } \\
\hline & $\begin{array}{l}\text { GGA- } \\
\text { VTG- }\end{array}$ & $\begin{array}{l}\text { All: } \\
\text { Original: } \\
\text { DGPS: } \\
\text { Altitude: } \\
\text { Other: } \\
\text { HDOP: }\end{array}$ & \\
\hline
\end{tabular}


Table 7. Summary of quality assessment messages for boat velocity.-Continued

\begin{tabular}{|l|l|l|l|}
\hline Type & Reference & Filter & Message \\
\hline Warning & BT- & All: & Interpolated discharge for invalid ensembles in a transect exceeds 25\%. \\
& & Original: & \\
& & ErrorVel: & \\
& & VertVel: & \\
& Other: & \\
& & 3Beams: & \\
\cline { 3 - 3 } & GGA- & All: & \\
& VTG- & Original: & \\
& DGPS: & \\
& & Altitude: & \\
& & Other: & \\
& HDOP: & \\
\hline
\end{tabular}

Table 8. Summary of quality assessment messages for water velocity.

\begin{tabular}{|l|l|l|l|}
\hline Type & Reference & Filter & Message \\
\hline Caution & wt- & All: & The percentage of invalid ensembles in a transect exceeds 5\%. \\
& & Original: & \\
& & ErrorVel: & \\
& & VertVel: & \\
& & Other: & \\
& & 3Beams: & \\
SNR: & All: & Interpolated discharge for consecutive invalid ensembles exceeds 3\%. \\
\hline Caution & wt- & Original: & \\
& & ErrorVel: & \\
& & VertVel: & \\
& & Other: & \\
& & 3Beams: & \\
& & SNR & \\
\hline Warning & WT- & All: & Interpolated discharge for consecutive invalid ensembles exceeds 5\%. \\
& & Original: & \\
& & ErrorVel: & \\
& & VertVel: & \\
& & Other: & \\
& & 3Beams: & \\
& & SNR: & \\
\hline Warning & WT- & All: & Interpolated discharge for invalid ensembles in a transect exceeds $25 \%$. \\
& & Original: & \\
& & ErrorVel: & \\
& & VertVel: & \\
& & Other: & \\
& & 3Beams: & \\
& & SNR: & \\
& &
\end{tabular}




\section{Extrapolation}

The quality of the extrapolation is based on the extrapolation uncertainty (see "Uncertainty Computation" section for details on estimating the extrapolation uncertainty). The logic for assessing the extrapolation quality is provided in figure 18.

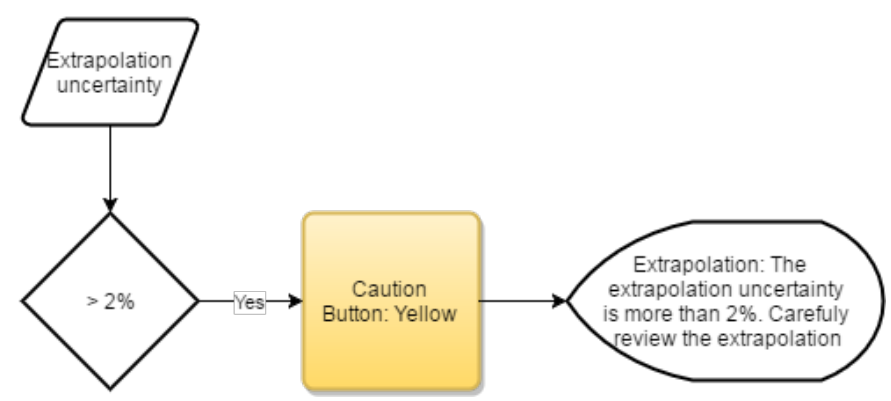

Figure 18. Flowchart of extrapolation assessment.

\section{Edges}

The quality assessment of edges is a combination of USGS policy, reasonableness of edge estimates, and consistency. The USGS policy requires supporting documentation or data for any edge estimate that exceeds 5 percent of the total discharge (Mueller and others, 2013). In addition, edges should not have a zero discharge and the edge type and sign (direction) should be consistent. The logic for assessing the quality of the edges is provided in figure 19.

\section{Uncertainty Computation}

Computing the uncertainty of an ADCP moving-boat discharge measurement is a complex task. Although many researchers have proposed approaches to determining the uncertainty of an ADCP moving-boat discharge measurement, an uncertainty model that has been generally accepted and that can be applied to actual field measurements does not exist. The method used in QRev is based on simple assumptions and what are likely the largest error sources. The approach used is neither detailed nor comprehensive but is presented as a guide to the user in rating the measurement.

QRev assesses the uncertainty based on the following six categories:

1. random,

2. invalid data,

3. edge discharge,

4. moving bed,

5. extrapolation, and

6. systematic.

The approach used to assign the uncertainty to each of these categories is discussed in the following sections. 


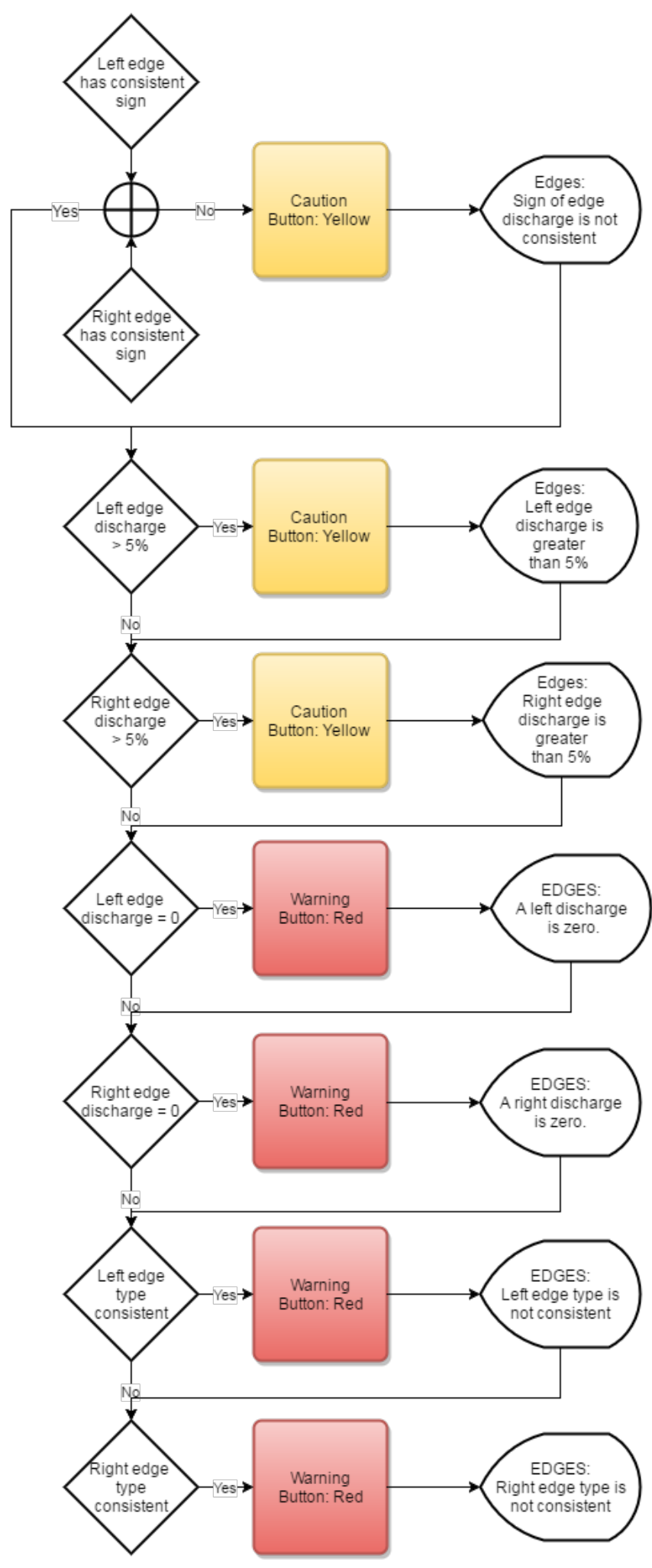

Figure 19. Flowchart of edges assessment. 


\section{Random Uncertainty}

The 95 percent random uncertainty expands the discharge coefficient of variation (QCOV) to a 95 percent level by applying a coverage factor from the Student's t-distribution based on the number or degrees of freedom and then dividing by the square root of the number of transects. When only two transects comprise a measurement, the theoretical Student's t approach is abandoned and the 95 percent uncertainty is computed as the QCOV *3.3.

\section{Invalid Data Uncertainty}

The 95 percent uncertainty for invalid data is assumed to be 20 percent of the percent discharge for invalid cells and ensembles.

\section{Edge Discharge Uncertainty}

The 95 percent uncertainty for the edge discharge is assumed to be 30 percent of the total discharge in the edges. This uncertainty accounts for uncertainty in the edge shape, roughness, distance to shore, depth, and velocity.

\section{Extrapolation Uncertainty}

The percent extrapolation uncertainty is determined by computing the percent difference in discharge from the selected extrapolation method to other possible extrapolation methods and averaging the best four options. The following is a list of the possible extrapolation methods:

1. top power, bottom power, 1/6th exponent;

2. top power, bottom power, optimized exponent;

3. top constant, bottom no slip, 1/6th exponent;

4. top constant, bottom no slip, optimized exponent;

5. top three point, bottom no slip, 1/6th exponent; and

6. top three point, bottom no slip, optimized exponent.

\section{Moving-Bed Test Uncertainty}

If bottom track is not the navigation reference, the percent moving-bed test uncertainty is set to zero. If bottom track is used and a moving-bed test is valid, the percent moving-bed test uncertainty is set to 1 percent if the test indicates no moving bed is present and to 1.5 percent if a moving bed is present. If the moving-bed test is invalid or not completed, the uncertainty is set to 3 percent.

\section{Systematic Uncertainty}

Systematic uncertainty is assumed to be 1.5 percent.

\section{Estimated 95 Percent Uncertainty}

The standard approach to computing the 95 percent uncertainty is to compute the square root of the sum of the squares of the standard deviations of the various sources of uncertainty (assuming independence) and then multiply by two to get a 95 percent value (Joint Committee for Guides in Metrology, 2008). So that the random uncertainty receives the proper coverage factor for the number of transects, each of the categories is estimated at the 95 percent level. The 
values are then each divided by two before taking the square root of the sum of the squares. Finally, the result is multiplied by two to achieve a 95 percent uncertainty for the measurement. The final value should not be viewed as strictly quantitative but more as a qualitative guide. The algorithms for the various sources of uncertainty are based on approximations and simple assumptions.

\section{User Override}

QRev allows the user to override the automatic uncertainty values by entering a different value. The user value for that category will then be used to compute the final uncertainty.

\section{Data File Formats}

QRev imports data from TRDI WinRiver II and SonTek RiverSurveyor Live. QRev can read the raw data files $(*$.mmt and *.pd0) produced by WinRiver II for all TRDI ADCPs. The raw data format for SonTek *.riv and *.rivr files is not available; therefore, QRev can only use the *.mat files produced by RiverSurveyor Live. Versions of RiverSurveyor Live prior to version 3.81 produced *.mat files that cannot be read by Matlab versions 2014 or later.

QRev's internal data storage format is defined in appendix 1. This format maintains the original data. The data are processed and then stored in a standardized format so that computational and filtering algorithms can be independent of the ADCP used to collect the data. The data can be saved in a Matlab file, which QRev can read for future review or processing.

QRev also produces an XML output file that can be used to import QRev processed results into the USGS SVMobile software or other agency databases. The XML format is defined in appendix 2.

\section{Summary and Need for Further Development}

QRev provides common and consistent computational algorithms combined with automated filtering and quality assessment of the data that substantially improves the quality and efficiency of streamflow measurements and helps ensure that U.S. Geological Survey (USGS) streamflow measurements are consistent, accurate, and independent of the manufacturer of the instrument used to make the measurement. QRev represents a substantial step towards standard processing algorithms and quality assessment that is instrument independent; however, additional research and development are needed. This research and development should improve interpolation and filter algorithms, provide more complex quality assessments, improve and standardize methods for estimating the discharge in unmeasured areas, and provide a more robust approach to estimating the uncertainty of a measurement. In addition, the data structure used in QRev serves as a good starting point for developing a standard data format that would be common among all hydroacoustic instrumentation. 


\section{References}

Chen, Cheng-Lung, 1989, Power law of flow resistance in open channels-Manning's formula revisited, in International Conference on Channel Flow and Catchment Runoff, Charlottesville, Va., May 22-26, 1989, Proceedings: Charlottesville, Va., Centennial of Manning's Formula and Kuichling's Rational Formula, p. 817-848.

Cleveland, W.S., 1979, Robust locally weighted regression and smoothing scatterplots: Journal of the American Statistical Association, v. 74, no. 368, p. 829-836, accessed April 6, 2016, at http://www.stat.washington.edu/courses/stat527/s13/readings/Cleveland_JASA_1979.pdf.

Cleveland, W.S., and Devlin, S.J., 1988, Locally weighted regression-An approach to Regression analysis by local fitting: Journal of the American Statistical Association v. 83, no. 403, p. 596-610, accessed April 6, 2016, at http://www.stat.washington.edu/courses/stat527/s13/readings/Cleveland_Delvin_JASA_1988. pdf.

Fulford, J.M., and Sauer, V.B., 1986, Comparison of velocity interpolation methods for computing open-channel discharge, in Subitsky, S.Y., ed., Selected papers in the hydrologic sciences: U.S. Geological Survey Water-Supply Paper 2290, p. 139-144, accessed April 6, 2016, at http://pubs.usgs.gov/wsp/wsp2290/.

Hagan, Ross E., 1989, Measuring discharge with current meters: International Irrigation Center, Utah State University, Logan, Utah, 39 p.

Joint Committee for Guides in Metrology, 2008, Evaluation of measurement data - Guide to the expression of uncertainty in measurement (GUM 1995 with minor corrections): Geneva, Switzerland.

Khan, M.A., Mahmood, K., and Skogerboe, G.V., 1997, Current meter discharge measurements for steady and unsteady flow conditions in irrigation channels: IWMI Pakistan Report T-007, International Irrigation Management Institute, Pakistan National Program.

Mueller, D.S., 2013, extrap-Software to assist the selection of extrapolation methods for moving-boat ADCP streamflow measurements: Computers \& Geosciences, v. 54, p. 211-218, accessed April 6, 2016, at http://www.sciencedirect.com/science/article/pii/S009830041300037X.

Mueller, D.S., 2015, Velocity bias induced by flow patterns around ADCPs and associated deployment platforms in Current, Waves and Turbulence Measurement Workshop, 11th, St. Petersburg, Fla., March 2-6, 2015, Proceedings: New York, IEEE, 7 p., accessed April 6, 2016, at http://ieeexplore.ieee.org/xpl/articleDetails.jsp?arnumber=7098103\&filter\%3DAND\%28p_IS Number\%3A7098093\%29.

Mueller, D.S., 2016, QRev-Software for computation and quality assurance of acoustic Doppler current profiler moving-boat streamflow measurements-User's manual for version 2.8: U.S. Geological Survey Open-File Report 2016-1052, 56 p.

Mueller, D.S., Abad, J.D., García, C.M., Gartner, J.W., García, M.H., and Oberg, K.A., 2007, Errors in acoustic Doppler profiler velocity measurements caused by flow disturbance: Journal of Hydraulic Engineering, v. 133, no. 12, p. 1411-1420, accessed April 6, 2016, at http://ascelibrary.org/doi/abs/10.1061/\%28ASCE\%2907339429\%282007\%29133\%3A12\%281411\%29. 
Mueller, D.S., Wagner, C.R., Rehmel, M.S., Oberg, K.A, and Rainville, Francois, 2013, Measuring discharge with acoustic Doppler current profilers from a moving boat: U.S. Geological Survey Techniques and Methods, book 3, chap. A22, 95 p., accessed April 6, 2016, at https://pubs.usgs.gov/tm/3a22/.

National Marine Electronics Association, 2002, NMEA 0183-Standard for interfacing marine electronic devices, version 3.01: National Marine Electronics Association, 88 p., accessed April 6, 2016, at http://www.plaisance-pratique.com/IMG/pdf/NMEA0183-2.pdf.

Oberg, K.A., and Schmidt, A.R., 1994, Measurements of leakage from Lake Michigan through three control structures near Chicago, Illinois, April-October 1993: U.S. Geological Survey Water-Resources Investigations Report 94-4112, 48 p., accessed April 6, 2016, at https://pubs.er.usgs.gov/publication/wri944112.

Office of Surface Water, 2013, Policy on required minimum screening distance for the RiverSurveyor M9: U.S. Geological Survey, Office of Surface Water Technical Memorandum 2014.02, 6 p., accessed April 6, 2016, at http://water.usgs.gov/admin/memo/SW/sw1402.pdf.

Rantz, S.E., and others, 1982, Measurement and computation of streamflow_Volume 1, Measurement of stage and discharge: U.S. Geological Survey Water-Supply Paper 2175, 284 p., accessed April 6, 2016, at http://pubs.usgs.gov/wsp/wsp2175/.

Schlichting, H., 1979, Boundary layer theory (7th ed.): New York, McGraw-Hill.

Seo, I., and Baek, K., 2004, Estimation of the longitudinal dispersion coefficient using the velocity profile in natural streams: American Society of Civil Engineers, Journal of Hydraulic Engineering, v. 130, no. 3, p. 227-236, accessed April 6, 2016, at http://ascelibrary.org/doi/abs/10.1061/(ASCE)0733-9429(2004)130\%3A3(227).

Simpson, M.R., 2002, Discharge measurements using a broad-band acoustic Doppler current profiler: U.S. Geological Survey Open-File Report 01-01, 123 p., accessed April 6, 2016, at http://pubs.usgs.gov/of/2001/ofr0101/.

Simpson, M.R., and Oltmann, R.N., 1993, Discharge-measurement system using an acoustic Doppler current profiler with applications to large rivers and estuaries: U.S. Geological Survey Water-Supply Paper 2395, 32 p., accessed April 6, 2016, at http://pubs.usgs.gov/wsp/wsp2395/.

SonTek, 2003, Principles of river discharge measurement: San Diego, Calif., SonTek, A Xylem Brand, $6 \mathrm{p}$.

SonTek, 2015, RiverSurveyor S5/M9 system manual firmware version 3.81: SonTek, San Diego, Calif., 162 p.

Teledyne RD Instruments, 1998, ADCP coordinate transformation-Formulas and calculations: San Diego, Calif., Teledyne RD Instruments, P/N 951-6079-00, 29 p.

Teledyne RD Instruments, 2007, Workhorse Rio Grande acoustic Doppler current profiler technical manual: San Diego, Calif., Teledyne RD Instruments, P/N 957-6241-00, 254 p., accessed April 6, 2016, at http://www.commtec.com/Prods/mfgs/RDI_Rivers/Rio\%20Grande\%20Technical\%20Manual_Nov07.pdf. Teledyne RD Instruments, 2014 , WinRiver II user's guide: San Diego, Calif., Teledyne RD Instruments, P/N 957-6231-00, 298 p.

Wagner, C.R., and Mueller, D.S., 2011, Comparison of bottom-track to global positioning system referenced discharges measured using an acoustic Doppler current profiler: Journal of Hydrology, v. 401, p. 250-258. 


\section{Appendix 1. Internal Data Format}

Explanation of data types and formatting:

clsClassName is the name of the class from which the object is created

objectName is the name of the object

variableName is a variable or object property

The following provides an overview of the top two levels of data stored by QRev.

messages: Variable for messages to user

version: Version number for QRev

meas: object of clsMeasurement

contains all the objects, methods, and variables associated with a measurement

stationName: name of location of measurement

stationNumber: station number of measurement

processing: sets the processing algorithms (SonTek, TRDI, QRev)

comments: notes from mmt file and comments entered by user in QRev

extTempChk: structure that stores manual and ADCP temperature check data

adcp: ADCP temperature recorded by user at time of external check

user: user recorded temperature from independent sensor

initialSettings: data structure with the settings as originally loaded from the manufacturer

transects: object of clsTransectData

contains all the objects and variables associated with each transect and associated methods

mbTests: object of clsMovingBedTests

contains moving-bed test data, quality evaluation methods, and results

sysTest: object of clsPreMeasurement

contains time stamp and output from system test

compassCal: object of clsPreMeasurement

contains time stamp and output from compass calibration

compassEval: object of clsPreMeasurement

contains time stamp and output from compass evaluation

extrapFit: object of clsComputeExtrap

contains all of the normalized data and methods for selecting an extrapolation method

discharge: object of clsQComp

contains the methods and resulting computed discharges

qa: object of clsQAData

contains the methods and results of the quality assurance checks

Full details of each object follows. 
transects: object of clsTransectData

contains all the objects and variables associated with each transect and associated methods.

filename: filename of transect data file

checked: transect was checked for use in the mmt file; assumed checked for SonTek

inTransectldx: index of ensemble data associated with the moving-boat portion of the transect

startEdge: starting edge of transect looking downstream (Left or Right)

adcp: object of clsInstrumentData

contains specific information about the ADCP used to collect the transect

wVel: object of clsWaterData

contains all the water velocity data and associated methods

boatVel: object of clsBoatStructure

contains all the boat velocity data and associated methods

gps: object of clsGPSData

contains all the GPS data and methods to compute boat position and velocity

sensors: object of clsSensors

contains objects for the various sensors and speed of sound

depths: object of clsDepthStructure

contains all the depth data and associated methods

edges: object of clsEdges

contains the data for edge discharge estimates

extrap: object of clsExtrapData

contains the extrapolation method and exponent for computing top and bottom extrapolation

dateTime: object of clsDateTime

contains all time associated data for the transect

The details for the various class in clsTransectData are presented after the classes contained in clsMeasurement. 
mbTests: object of clsMovingBedTests

contains moving-bed test data, quality evaluation methods, and results

type: Loop or Stationary

duration_sec: duration of test in secs

percentInvalidBT: percent of invalid bottom track

compassDiff_deg: difference in heading for out and back of loop

flowDir_deg: mean flow direction from loop test

mbDir_deg: moving bed or closure error direction

distUS_m: distance moved upstream in meters

flowSpd_mps: magnitude of water velocity in meters per second

mbSpd_mps: magnitude of moving-bed velocity in meters per second

percentMB: potential error caused by a moving bed in percent

movingBed: moving bed determined ('Yes' or 'No')

userValid: logical to allow user to determine if test should be considered a valid test

testQuality: quality of test ('Valid', 'Warnings', 'Invalid')

use2Correct: use this test to correct discharge

selected: selected as valid moving-bed test to use for correction or determining moving-bed

condition

messages: cell array of warning and error messages based on data processing

nearBedSpeed_mps: mean near-bed water speed for test in meters per second

stationaryUSTrack: upstream component of the bottom track referenced ship track

stationaryCSTrack: cross-stream component of the bottom track referenced ship track

stationaryMBVel: moving-bed velocity by ensemble

transect: object of clsTransectData

sysTest: object of clsPreMeasurement

contains time stamp and output from system test

timestamp: time stamp of test

data: data from text, typically all text

compassCal: object of clsPreMeasurement

contains time stamp and output from compass calibration

timestamp: time stamp of test

data: data from text, typically all text

compassEval: object of clsPreMeasurement

contains time stamp and output from compass evaluation

timestamp: time stamp of test

data: data from text, typically all text 
extrapFit: object of clsComputeExtrap

threshold: threshold as a percent for determining if a median is valid

subsection: percent of discharge

fitMethod: method used to determine fit (Automatic or Manual)

messages: variable for messages to user

normData: object of class clsNormData

fileName: name of transect file

cellDepthNormalized: normalized depth of cell

unitNormalized: normalized discharge or velocity for all depth cells

unitNormalizedMed: median of normalized data within 5 percent partitions

unitNormalizedNo: number of data points in each median

unitNormalizedz: relative depth for each median (5 percent increments)

unitNormalized25: value for which 25 percent of normalized values are smaller

unitNormalized75: value for which 25 percent of normalized values are larger

dataType: type of data (velocity or discharge)

dataExtent: extents of data defined by user input subsection percentages

validData: index of median values with point count greater than threshold cutoff

selFit: object of class clsSelectFit

filename: name of transect file

topMethod: top extrapolation method

botMethod: bottom extrapolation method

coef: power fit coefficient

exponent: power fit exponent

$u$ : fit values of the variable

uAuto: fit values from automatic fit

$\mathrm{z}$ : distance from the streambed for fit variable

zAuto: $z$ values for automatic fit

expMethod: method to determine exponent (default, optimize, or manual)

dataType: type of data (velocity or unit discharge)

exponent95confint: 95 percent confidence intervals for optimized exponent

residuals: residuals from fit

rsqr: adjusted $r^{\wedge} 2$ for optimized exponent

fitMethod: user selected method (Automatic or Manual)

botMethodAuto: selected extrapolation for top

topMethodAuto: selected extrapolation for bottom

exponentAuto: selected exponent

topfitr2: top fit custom coefficient of determination

topmaxdiff: maximum difference between power and three point at top

botdiff: difference between power and no slip at 10 percent of the depth from the bottom

botrsqr: bottom fit coefficient of determination

fitrsqr: selected fit of selected power/no slip fit

nsexponent: no slip optimized exponent 


\author{
ppexponent: "Power, power" fit optimized exponent \\ topr2: coefficient of determination for linear fit through top four median cells \\ qSensitivity: object of class clsExtrapQSensitivity \\ qPPmean: discharge "power, power" with 1/6 exponent \\ qPPoptmean: discharge "power, power" optimized \\ qCNSmean: discharge "constant, no slip with 1/6 exponent" \\ qCNSoptmean: discharge "constant, optimized no slip" \\ q3pNSmean: discharge "three point, no slip with 1/6 exponent" \\ q3pNSoptmean: discharge "three point, optimized no slip" \\ qPPoptperdiff: "power, power" fit optimized percent difference from "power, power" with 1/6 \\ exponent \\ qCNSperdiff: "constant, no slip with 1/6 exponent" percent difference from "power, power" \\ with $1 / 6$ exponent \\ qCNSoptperdiff: "constant, optimized no slip" percent difference from "power, power" with \\ $1 / 6$ exponent \\ q3pNSperdiff: "three point, no slip with $1 / 6$ exponent" percent difference from "power, \\ power" with $1 / 6$ exponent \\ q3pNSoptperdiff: "three point, optimized no slip" percent difference from "power, power" \\ with $1 / 6$ exponent \\ ppExponent: optimized "power, power" exponent \\ nsExponent: optimized no slip exponent \\ manTop: manually specified top method \\ manBot: manually specified bottom method \\ manExp: manually specified exponent \\ qManmean: mean discharge for manually specified extrapolations \\ qManperdiff: manually specified extrapolations percent difference from "power, power" with \\ $1 / 6$ exponent
}

\title{
discharge: object of clsQComp
}

contains the methods and resulting computed discharges

top: transect total extrapolated top discharge

middle: transect total measured middle discharge including interpolations

bottom: transect total extrapolated bottom discharge

topEns: rxtrapolated top discharge by ensemble

middleCells: measured middle discharge including interpolations by cell

middleEns: measured middle discharge including interpolations by ensemble

bottomEns: extrapolate bottom discharge by ensemble

left: left edge discharge

leftidx: ensembles used for left edge

right: right edge discharge

rightidx: ensembles used for right edge

totalUncorrected: total discharge for transect uncorrected for moving bed, if required 
total: total discharge with moving-bed correction applied if necessary

correctionFactor: moving-bed correction factor, if required

intCells: total discharge computed for invalid depth cells excluding invalid ensembles

intEns: total discharge computed for invalid ensembles

\section{uncertainty: object of clsUncertainty}

cov: coefficient of variation for all used transect discharges

cov95: coefficient of variation inflated by the 95 percent coverage factor

invalid95: estimated 95 percent uncertainty for discharge in invalid bins and ensembles

edges95: estimated 95 percent uncertainty for the computed edge discharges

extrapolation95: estimated 95 percent uncertainty in discharge because of top and bottom

extrapolations

movingBed95: estimated 95 percent uncertainty caused by a moving-bed tests and conditions

systematic: systematic error estimated at 1.5 percent

total95: estimated 95 percent uncertainty in discharge using automated values

cov95User: user provided value for random uncertainty

invalid95User: user provided estimate of uncertainty for invalid data

edges95User: user provided estimate of uncertainty for edges

extrapolation95User: user provided estimate of uncertainty for top and bottom extrapolation

movingBed95User: user provided estimate of uncertainty caused by a moving-bed conditions

systematicUser: user provided estimate of systematic uncertainty

total95User: estimated 95 percent uncertainty in discharge using user provide values to override

automated values

qa: object of clsQAData

qRunThresholdCaution: caution threshold for interpolated discharge for a run of invalid ensembles, in percent

qRunThresholdWarning: warning threshold for interpolated discharge for a run of invalid ensembles, in percent

qTotalThresholdWarning: warning threshold for total interpolated discharge for invalid ensembles, in percent

ensThresholdCaution: caution threshold for total number of invalid ensembles, in percent

transects: data structure for quality assurance checks of transects

messages: quality assessment messages to the user

status: overall status, good, caution, or warning

uncertainty: code for only two transects (0-good, 1-caution)

duration: code for check that duration is $>720 \mathrm{sec}$ (0-good, 1-caution)

number: code for the number of transects to use (0-good, 1-caution, 2-warning)

recip: code for reciprocal transects (0-good, 2-warning)

sign: code for consistent sign in total discharge (0-good, 2-warning)

systemTest: data structure for quality assurance checks of system tests

messages: quality assessment messages to the user 
status: overall status, good, caution, or warning

nFailedTests: number of failed system tests

validTests: logical of valid system tests

compass: data structure for quality assurance checks of compass tests and evaluations

messages: quality assessment messages to the user

status: overall status, good, caution, or warning

temperature: data structure for quality assurance checks of temperature comparisons and change messages: quality assessment messages to the user

status: overall status, good, caution, or warning

movingbed: data structure for quality assurance checks of moving-bed tests and conditions

messages: quality assessment messages to the user

code: quality code, 1-Good, 2-Caution, 3-Warning

status: overall status, good, caution, or warning

user: data structure for quality assurance checks of user input data

messages: quality assessment messages to the user

status: overall status, good, caution, or warning

draft: draft consistency and zero value check (0-good, 1-caution, 2-warning)

magvar: magvar consistency and zero value check (0-good, 1-caution, 2-warning)

staName: checks for a station name (0-good, 1-caution)

staNumber: checks for a station number (0-good, 1-caution)

depths: data structure for quality assurance checks of depth data

messages: quality assessment messages to the user

status: overall status, good, caution, or warning

qTotal: total interpolated discharge in invalid ensembles, cubic meters per second

qMaxRun: maximum interpolated discharge in a continuous run of invalid ensembles, cubic

meters per second

ensInvalidCaution: logical array indicating what transects and tests exceed the

ensThresholdCaution. Each transect as a row and each filter as a column

qRunCaution: logical array indicating what transects and tests exceed the

qRunThresholdCaution. Each transect as a row and each filter as a column

qTotalCaution: logical array indicating what transects and tests exceed the

qTotalThresholdCaution. Each transect as a row and each filter as a column

qRunWarning: logical array indicating what transects and tests exceed the

qRunThresholdWarning. Each transect as a row and each filter as a column

btVel: data structure for quality assurance checks of bottom track velocities

messages: quality assessment messages to the user

status: overall status, good, caution, or warning

qTotal: total interpolated discharge in invalid ensembles, cubic meters per second

qMaxRun: maximum interpolated discharge in a continuous run of invalid ensembles, cubic

meters per second

ensInvalidCaution: logical array indicating what transects and tests exceed the

ensThresholdCaution. Each transect as a row and each filter as a column 
qRunCaution: logical array indicating what transects and tests exceed the

qRunThresholdCaution. Each transect as a row and each filter as a column

qTotalCaution: logical array indicating what transects and tests exceed the

qTotalThresholdCaution. Each transect as a row and each filter as a column

qRunWarning: logical array indicating what transects and tests exceed the

qRunThresholdWarning. Each transect as a row and each filter as a column

ggaVel: data structure for quality assurance checks of GGA boat velocities

messages: quality assessment messages to the user

status: overall status, good, caution, or warning

qTotal: total interpolated discharge in invalid ensembles, cubic meters per second

qMaxRun: maximum interpolated discharge in a continuous run of invalid ensembles, cubic meters per second

ens/nvalidCaution: logical array indicating what transects and tests exceed the ensThresholdCaution. Each transect as a row and each filter as a column

qRunCaution: logical array indicating what transects and tests exceed the

qRunThresholdCaution. Each transect as a row and each filter as a column

qTotalCaution: logical array indicating what transects and tests exceed the

qTotalThresholdCaution. Each transect as a row and each filter as a column

qRunWarning: logical array indicating what transects and tests exceed the

qRunThresholdWarning. Each transect as a row and each filter as a column

vtgVel: data structure for quality assurance checks of VTG boat velocities

messages: quality assessment messages to the user

status: overall status, good, caution, or warning

qTotal: total interpolated discharge in invalid ensembles, cubic meters per second

qMaxRun: maximum interpolated discharge in a continuous run of invalid ensembles, cubic

meters per second

ensInvalidCaution: logical array indicating what transects and tests exceed the

ensThresholdCaution. Each transect as a row and each filter as a column

qRunCaution: logical array indicating what transects and tests exceed the

qRunThresholdCaution. Each transect as a row and each filter as a column

qTotalCaution: logical array indicating what transects and tests exceed the

qTotalThresholdCaution. Each transect as a row and each filter as a column

qRunWarning: logical array indicating what transects and tests exceed the

qRunThresholdWarning. Each transect as a row and each filter as a column

wVel: data structure for quality assurance checks of water track velocities

messages: quality assessment messages to the user

status: overall status, good, caution, or warning

qTotal: total interpolated discharge in invalid ensembles, cubic meters per second

qMaxRun: maximum interpolated discharge in a continuous run of invalid ensembles, cubic meters per second

ensInvalidCaution: logical array indicating what transects and tests exceed the ensThresholdCaution. Each transect as a row and each filter as a column 
qRunCaution: logical array indicating what which transects and tests exceed the qRunThresholdCaution. Each transect as a row and each filter as a column qTotalCaution: logical array indicating what transects and tests exceed the

qTotalThresholdCaution. Each transect as a row and each filter as a column qRunWarning: logical array indicating what transects and tests exceed the

qRunThresholdWarning. Each transect as a row and each filter as a column extrapolation: data structure for quality assurance checks of extrapolations messages: quality assessment messages to the user status: overall status, good, caution, or warning edges: data structure for quality assurance checks of edge discharge estimates messages: quality assessment messages to the user status: overall status, good, caution, or warning rightSign: discharge sign of right edge not consistent, caution (1) leftSign: discharge sign of left edge not consistent, caution (1) leftzero: left edge with zero discharge, warning (2) rightzero: right edge with zero discharge, warning (2) leftType: left edge type is inconsistent, warning (2) rightType: right edge type is inconsistent, warning (2) leftQ: left edge discharge is greater than 5 percent, caution (1) rightQ: right edge discharge is greater than 5 percent, caution (1) 
Objects in clsTransectData.

adcp: object of clsInstrumentData

serialNum: serial number of ADCP

manufacturer: manufacturer of ADCP (SonTek, TRDI)

model: model of ADCP (Rio Grande, StreamPro, RiverRay, M9, S5)

firmware: firmware version

frequency_hz: frequency of ADCP (could be "Multi")

beamAngle_deg: angle of beam from vertical

beamPattern: pattern of beams (concave or convex)

configurationCommands: configuration commands sent to ADCP

tMatrix: object of clsTransformationMatrix

source: source of matrix (Nominal, ADCP)

matrix: transformation matrix, $4 \times 4$ matrix for TRDI, $4 \times 4 \times 3$ or 2 for SonTek.

\section{wVel: object of clsWaterData}

rawVel_mps: contains the raw unfiltered velocity data in meters per second. Rows 1-4 are beams

$1,2,3$, and 4 if beam or $u, v, w$, and $d$ if otherwise

frequency: defines ADCP frequency used for velocity measurement

origCoordSys: defines the original raw data velocity coordinate system "Beam", "Inst", "Ship",

"Earth"

origNavRef: defines the original raw data navigation reference: "None", "BT", "GGA", "VTG"

corr: correlation values for WT, if available

rssi: returned acoustic signal strength.

rssiUnits: units for returned acoustic signal strength: "Counts", "dB", "SNR"

waterMode: water mode for TRDI or 'Variable' for SonTek

blankingDistance_m: distance below transducer where data are marked invalid because of

potential ringing interference

cellsAboveSL: logical array of depth cells above the side lobe cutoff based on selected depth

reference

cellsAboveSLbt: logical array of depth cells above the side lobe cutoff based on BT

s/LagEffect_m: side lobe distance caused by lag and transmit length

uEarthNoRef_mps: horizontal velocity in $\mathrm{x}$-direction with no boat referenced applied, in meters per second

vEarthNoRef_mps: horizontal velocity in y-direction with no boat referenced applied, in meters per second

u_mps: horizontal velocity in $\mathbf{x}$-direction, in meters per second

$v \_m p s:$ horizontal velocity in $y$-direction, in meters per second

uProcessed_mps: horizontal velocity in $\mathrm{x}$-direction filtered and interpolated

$v$ Processed_mps: horizontal velocity in $\mathrm{y}$-direction filtered and interpolated

w_mps: vertical velocity (+ up), in meters per second

d_mps: difference in vertical velocities compute from opposing beam pairs, in meters per second 
invalidIndex: index of ensembles with no valid raw velocity data

num/nvalid: estimated number of depth cells in ensembles with no valid raw velocity data

validData: 3-dimensional logical array of valid data

Dim3 1-composite

Dim3 2-original, cells above side lobe

Dim3 3-dFilter

Dim3 4-wFilter

Dim3 5-smoothFilter

Dim3 6-beamFilter

Dim3 7-excludedFilter

Dim3 8-snrFilter

Dim3 9-validDepthFilter

beamFilter: 3 for three-beam solutions, 4 for four-beam solutions

dFilter: difference velocity filter "Auto", "Manual", "Off"

dFilterThreshold: threshold for difference velocity filter

wFilter: vertical velocity filter "Auto", "Manual", "Off"

wFilterThreshold: threshold for vertical velocity filter

excludedDist: distance below transducer above which data are marked invalid

smoothFilter: filter based on smoothing function "Auto", "Off"

smoothSpeed: smoothed boat speed

smoothUpperLimit: smooth function upper limit of window

smoothLowerLimit: smooth function lower limit of window

snrFilter: signal to noise ratio filter for SonTek data

snrRng: range of beam averaged signal to noise ratio

wtDepthFilter: water track in ensembles with invalid depth are marked invalid

interpolateEns: type of interpolation: "None", “ExpandedT", “Hold9", "HoldLast", "Linear", "TRDI"

interpolateCells: type of interpolation: "None", "TRDI", "Linear"

coordSys: defines the velocity coordinate system "Beam", "Inst", "Ship", "Earth"

navRef: defines the navigation reference: "None", "BT", "GGA", "VTG"

s/CutoffPer: percentage of range to mark invalid caused by side lobe interference

slCutoffNum: number of user specified cells to mark invalid instead of using percentage

slCutoffType: type of side lobe cutoff used "Percent" or user specified number of cells "Number"

boatVel: object of clsBoatStructure

selected: (string) name of the object of clsBoatData to be used for discharge computations

composite: composite tracks On or Off

btVel: object of clsBoatData

rawVel_mps: contains the raw unfiltered velocity data in meters per second. Rows 1-4 are

beams 1, 2, 3, and 4 if beam or $u, v, w$, and $d$ if otherwise

frequency_Hz: defines ADCP frequency used for velocity measurement

origCoordSys: defines the original raw data velocity coordinate system "Beam", "Inst", "Ship",

"Earth" 
navRef: "BT"

coordSys: defines the current coordinate system "Beam", "Inst", "Ship", "Earth" for u, v, w, and

d

u_mps: horizontal velocity in $\mathrm{x}$-direction, in meters per second

$v \_m p s$ : horizontal velocity in $y$-direction, in meters per second

w_mps: vertical velocity (+ up), in meters per second

$d_{-} m p s$ : difference in vertical velocities compute from opposing beam pairs, in meters per second

numlnvalid: number of ensembles with invalid velocity data

bottomMode: bottom track mode for TRDI, 'Variable' for SonTek

uProcessed_mps: horizontal velocity in x-direction filtered and interpolated

$v$ Processed_mps: horizontal velocity in y-direction filtered and interpolated

processedSource: source of velocity: BT, VTG, GGA, INT

dFilter: difference velocity filter "Auto", "Manual", "Off"

dFilterThreshold: threshold for difference velocity filter

wFilter: vertical velocity filter "Auto", "Manual", "Off"

wFilterThreshold: threshold for vertical velocity filter

gpsDiffQualFilter: not applicable

gpsAltitudeFilter: not applicable

gpsAltitudeFilterChange: not applicable

gpsHDOPFilter: not applicable

gpsHDOPFilterMax: not applicable

gpsHDOPFilterChange: not applicable

smoothFilter: filter based on smoothing function "Auto", "Off"

smoothSpeed: smoothed boat speed

smoothUpperLimit: smooth function upper limit of window

smoothLowerLimit: smooth function lower limit of window

interpolate: type of interpolation: "None", “ExpandedT", "Hold9”, "HoldLast”, "Linear",

"Smooth"

beamFilter: 3 for three-beam solutions, 4 for four-beam solutions

validData: logical array of identifying valid and invalid data for each filter applied

Row 1-composite

Row 2-original

Row 3-dFilter or diffQual

Row 4-wFilter or altitude

Row 5-smoothFilter

Row 6-beamFilter or HDOP

\section{ggaVel: object of clsBoatData}

rawVel_mps: contains the raw unfiltered velocity data in meters per second. Rows 1-4 are beams $1,2,3$, and 4 if beam or $u, v, w$, and $d$ if otherwise

frequency_Hz: not applicable

origCoordSys: "Earth" 
navRef: "GGA"

coordSys: "Earth" for $\mathrm{u}, \mathrm{v}, \mathrm{w}$, and d

u_mps: horizontal velocity in $\mathrm{x}$-direction, in meters per second

$v \_m p s$ : horizontal velocity in $y$-direction, in meters per second

w_mps: vertical velocity (+ up), in meters per second

d_mps: difference in vertical velocities compute from opposing beam pairs, in meters per

second

numlnvalid: number of ensembles with invalid velocity data

bottomMode: not applicable

uProcessed_mps: horizontal velocity in $\mathrm{x}$-direction filtered and interpolated

$v$ Processed_mps: horizontal velocity in $\mathrm{y}$-direction filtered and interpolated

processedSource: source of velocity: BT, VTG, GGA, INT

dFilter: not applicable

dFilterThreshold: not applicable

wFilter: not applicable

wFilterThreshold: not applicable

gpsDiffQualFilter: differential correction quality $(1,2,4)$

gpsAltitudeFilter: change in altitude filter "Auto", "Manual", "Off"

gpsAltitudeFilterChange: threshold from mean for altitude filter

gpsHDOPFilter: horizontal dilution of precision filter "Auto", "Manual", "Off"

gpsHDOPFilterMax: max acceptable value of HDOP

gpsHDOPFilterChange: maximum change allowed from mean

smoothFilter: filter based on smoothing function "Auto", "Off"

smoothSpeed: smoothed boat speed

smoothUpperLimit: smooth function upper limit of window

smoothLowerLimit: smooth function lower limit of window

interpolate: type of interpolation: "None", “ExpandedT", “Hold9”, “HoldLast", "Linear",

"Smooth"

beamFilter: not applicable

validData: Logical array of identifying valid and invalid data for each filter applied

Row 1-composite

Row 2-original

Row 3-dFilter or diffQual

Row 4-wFilter or altitude

Row 5-smoothFilter

Row 6-beamFilter or HDOP

vtgVel: object of clsBoatData

rawVel_mps: contains the raw unfiltered velocity data in meters per second. Rows 1-4 are

beams 1, 2, 3, and 4 if beam or $u, v, w$, and d if otherwise

frequency_Hz: not applicable

origCoordSys: "Earth"

navRef: "VTG" 
coordSys: "Earth" for u, v, w, and d

u_mps: horizontal velocity in $\mathrm{x}$-direction, in meters per second

$v \_m p s$ : horizontal velocity in $y$-direction, in meters per second

w_mps: vertical velocity (+ up), in meters per second

d_mps: difference in vertical velocities compute from opposing beam pairs, in meters per second

numlnvalid: number of ensembles with invalid velocity data

bottomMode: not applicable

uProcessed_mps: horizontal velocity in x-direction filtered and interpolated

$v$ Processed_mps: horizontal velocity in y-direction filtered and interpolated

processedSource: source of velocity: BT, VTG, GGA, INT

dFilter: not applicable

dFilterThreshold: not applicable

wFilter: not applicable

wFilterThreshold: not applicable

gpsDiffQualFilter: differential correction quality $(1,2,4)$

gpsAltitudeFilter: change in altitude filter "Auto", "Manual", "Off"

gpsAltitudeFilterChange: threshold from mean for altitude filter

gpsHDOPFilter: horizontal dilution of precision filter "Auto", "Manual", "Off"

gpsHDOPFilterMax: max acceptable value of HDOP

gpsHDOPFilterChange: maximum change allowed from mean

smoothFilter: filter based on smoothing function "Auto", "Off"

smoothSpeed: smoothed boat speed

smoothUpperLimit: smooth function upper limit of window

smoothLowerLimit: smooth function lower limit of window

interpolate: type of interpolation: "None", "ExpandedT", "Hold9", “HoldLast", "Linear",

"Smooth"

beamFilter: not applicable

validData: logical array of identifying valid and invalid data for each filter applied

Row 1-composite

Row 2-original

Row 3-dFilter or diffQual

Row 4-wFilter or altitude

Row 5-smoothFilter

Row 6-beamFilter or HDOP

gps: object of clsGPSData

rawGGALat_deg: raw latitude in degrees, [n,ensemble]

rawGGALon_deg: raw longitude in degrees, [n,ensemble]

rawGGAAltitude_m: raw altitude in meters, [n,ensemble]

rawGGADifferential: differential correction indicator, [n,ensemble]

rawGGAHDOP: horizontal dilution of precision, [n,ensemble] 
rawGGAUTC: UTC time, hhmmss.ss, [n,ensemble]

rawGGASerialTime: UTC time of gga data in seconds past midnight, [n,ensemble]

rawGGANumSats: number of satellites reported in GGA sentence, [n,ensemble]

rawVTGCourse_deg: course in degrees, [n,ensemble]

rawVTGSpeed_mps: speed in meters per second, [n,ensemble]

rawVTGDeltaTime: VTG delta time (sec)

rawGGADeltaTime: GGA delta time (sec)

extGGALat_deg: raw latitude in degrees computed by external source, [1,ensemble]

extGGALon_deg: raw longitude in degrees computed by external source, [1,ensemble]

extGGAAltitude_m: raw altitude in meters computed by external source, [1,ensemble]

extGGADifferential: differential correction indicator computed by external source, [1,ensemble]

extGGAHDOP: horizontal dilution of precision computed by external source, [1,ensemble]

extGGAUTC: UTC time, hhmmss.ss computed by external source, [1,ensemble]

extGGASerialTime: UTC time of gga data in seconds past midnight computed by external source,

[1,ensemble]

extGGANumSats: number of satellites computed by external source [1,ensemble]

extVTGCourse_deg: course in degrees computed by external source, [1,ensemble]

extVTGSpeed_mps: speed in meters per second computed by external source, [1,ensemble]

ggaPositionMethod: method used to process gga data for position ('End', 'Average', 'External')

ggaVelocityMethod: method used to process gga data for velocity ('End', 'Average', 'External')

vtgVelocityMethod: method used to process vtg data for velocity ('Average', 'External)

ggaLatEns_deg: processed latitude in degrees, [1,ensemble]

ggaLonEns_deg: processed longitude in degrees, [1,ensemble]

UTMEns_m: UTM position from processed gga data, [2,ensemble]

ggaVelocityEns_mps: Boat velocity computed from gga data [2,ensemble]

ggaSerialTimeEns: UTC time of gga data in seconds past midnight, [1,ensemble]

vtgVelocityEns_mps: boat velocity computed from vtg data [2,ensemble]

perGoodEns: percentage of available data used to compute ensemble value

hdopEns: horizontal dilution of precision for each ensemble using velocity method

numSatsEns: number of satellites for each ensemble, using velocity method

altitudeEns_m: altitude for each ensemble, using velocity method

diffQualEns: differential quality for each ensemble, using velocity method

sensors: object of clsSensors

heading_deg: object of clsSensorStructure

selected: name of selected source "internal", "external"

internal: object of clsHeadingData

data: corrected heading data

orginalData: original uncorrected heading data

source: source of heading data (Internal, GPS, Gyro, Other)

magvar_deg: magnetic variation for these heading data

magvarOrig_deg: original magnetic variation 
alignCorrection_deg: alignment correction to align compass with instrument

external: object of clsHeadingData

data: corrected heading data

orginalData: original uncorrected heading data

source: source of heading data (Internal, GPS, Gyro, Other)

magvar_deg: magnetic variation for these heading data

magvarOrig_deg: original magnetic variation

alignCorrection_deg: alignment correction to align compass with instrument

user: not used for heading

pitch_deg: object of clsSensorStructure

selected: name of selected source "internal", "external", "user"

internal: object of clsSensorData

data: time series data for sensor

dataOrig: original time series data for sensor

source: source of data

external: object of clsSensorData

data: time series data for sensor

dataOrig: original time series data for sensor

source: source of data

user: object of clsSensorData

data: time series data for sensor

dataOrig: original time series data for sensor

source: source of data

roll_deg: object of clsSensorStructure

selected: name of selected source "internal", "external", "user"

internal: object of clsSensorData

data: time series data for sensor

dataOrig: original time series data for sensor

source: source of data

external: object of clsSensorData

data: time series data for sensor

dataOrig: original time series data for sensor

source: source of data

user: object of clsSensorData

data: time series data for sensor

dataOrig: original time series data for sensor

source: source of data

temperature_degC: object of clsSensorStructure

selected: name of selected source "internal", "external", "user"

data: time series data for sensor

dataOrig: original time series data for sensor

source: source of data 
external: object of clsSensorData

data: time series data for sensor

dataOrig: original time series data for sensor

source: source of data

user: object of clsSensorData

data: time series data for sensor

dataOrig: original time series data for sensor

source: source of data

salinity_ppt: object of clsSensorStructure

selected: name of selected source "internal", "external", "user"

internal: object of clsSensorData

data: time series data for sensor

dataOrig: original time series data for sensor

source: source of data

external: object of clsSensorData

data: time series data for sensor

dataOrig: original time series data for sensor

source: source of data

user: object of clsSensorData

data: time series data for sensor

dataOrig: original time series data for sensor

source: source of data

speedOfSound_mps: object of clsSensorStructure

selected: name of selected source "internal", "external", "user"

internal: object of clsSensorData

data: time series data for sensor

dataOrig: original time series data for sensor

source: source of data

external: object of clsSensorData

data: time series data for sensor

dataOrig: original time series data for sensor

source: source of data

user: object of clsSensorData

data: time series data for sensor

dataOrig: original time series data for sensor

source: source of data

depths: object of clsDepthStructure

selected: name of object of clsDepthData for compute $Q$

composite: turn composite depths "On" or "Off"

btDepths: object of clsDepthData

depthOrig_m: original multibeam depth data from transect file (includes draftOrig), in 
meters

depthBeams_m: depth data from transect file adjusted for any draft changes, in meters depthProcessed_m: depth data filtered and interpolated

depthFreq_Hz: defines ADCP frequency used of each raw data point

depthInvalidIndex: index of depths marked invalid

depthSource: source of depth data (BT, VB, DS)

depthSourceEns: source of each depth value

draftOrig_m: original draft from data files, in meters

draftUse_m: draft used in computation of depth_m and depthCellDepths_m

depthCellDepthOrig_m: depth cell range from the transducer, in meters

depthCellDepth_m: depth to centerline of depth cells, in meters

depthCellsize_m: size of depth cells, in meters

smoothDepth: smoothed beam depth

smoothUpperLimit: smooth function upper limit of window

smoothLowerLimit: smooth function lower limit of window

avgMethod: defines averaging method: "Simple", "IDW"

filterType: type of filter: "None", "TRDI", "Smooth"

interpType: type of interpolation: "None", "Linear", "Smooth"

validDataMethod: "QRev" requires two valid beams, "TRDI" requires three valid beams

validBeams: logical array, one row for each beam identifying valid data

validData: logical array of valid mean depth for each ensemble

vbDepths: object of clsDepthData

depthOrig_m: original depth data from transect file (includes draftOrig), in meters

depthBeams_m: depth data from transect file adjusted for any draft changes, in meters

depthProcessed_m: depth data filtered and interpolated

depthFreq_Hz: defines ADCP frequency used of each raw data point

depthInvalidIndex: index of depths marked invalid

depthSource: source of depth data (BT, VB, DS)

depthSourceEns: source of each depth value

draftOrig_m: original draft from data files, in meters

draftUse_m: draft used in computation of depth_m and depthCellDepths_meters

depthCellDepthOrig_m: depth cell range from the transducer, in meters

depthCellDepth_m: depth to centerline of depth cells, in meters

depthCellSize_m: size of depth cells, in meters

smoothDepth: smoothed beam depth

smoothUpperLimit: smooth function upper limit of window

smoothLowerLimit: smooth function lower limit of window

avgMethod: defines averaging method: "Simple", "IDW"

filterType: type of filter: "None", "TRDI", "Smooth"

interpType: type of interpolation: "None", "Linear", "Smooth"

validDataMethod: "QRev" requires two valid beams, "TRDI" requires three valid beams

validBeams: logical array, one row for each beam identifying valid data 
validData: logical array of valid mean depth for each ensemble

dsDepths: object of clsDepthData

depthOrig_m: original depth data from transect file (includes draftOrig), in meters

depthBeams_m: depth data from transect file adjusted for any draft changes, in meters depthProcessed_m: depth data filtered and interpolated

depthFreq_Hz: defines ADCP frequency used of each raw data point

depthInvalidIndex: index of depths marked invalid

depthSource: source of depth data (BT, VB, DS)

depthSourceEns: source of each depth value

draftOrig_m: original draft from data files, in meters

draftUse_m: draft used in computation of depth_m and depthCellDepths_m

depthCellDepthOrig_m: depth cell range from the transducer, in meters

depthCellDepth_m: depth to centerline of depth cells, in meters

depthCellSize_m: size of depth cells, in meters

smoothDepth: smoothed beam depth

smoothUpperLimit: smooth function upper limit of window

smoothLowerLimit: smooth function lower limit of window

avgMethod: defines averaging method: "Simple", "IDW"

filterType: type of filter: "None", "TRDI", "Smooth"

interpType: type of interpolation: "None", "Linear", "Smooth"

validDataMethod: "QRev" requires two valid beams, "TRDI" requires three valid beams

validBeams: logical array, one row for each beam identifying valid data

validData: logical array of valid mean depth for each ensemble

edges: object of clsEdges

recEdgeMethod: "Variable" uses SonTek's equation, "Fixed" uses 0.91

velMethod: "VectorProf" uses SonTek's method, "MeasMag" uses TRDI's method

left: object of clsEdgeData

type: type or shape of edge: "Triangular", "Square:", "Custom", "User Q"

dist_m: distance to shore

custCoef: discharge computation coefficient

numEns2Avg: number of ensembles to average for depth and velocity

userQ_cms: discharge provided directly from user

right: object of clsEdgeData

type: type or shape of edge: "Triangular", "Square:", “Custom”, "User Q"

dist_m: distance to shore

custCoef: discharge computation coefficient

numEns2Avg: number of ensembles to average for depth and velocity

userQ_cms: discharge provided directly from user

extrap: object of clsExtrapData

topMethodOrig: extrapolation method for top of profile: "Power", "Constant", "3-Point" 
botMethodOrig: extrapolation method for bottom of profile: "Power", "No Slip" exponentOrig: exponent for power of no slip methods

topMethod: extrapolation method for top of profile: "Power", "Constant", "3-Point" botMethod: extrapolation method for bottom of profile: "Power", "No Slip" exponent: exponent for power of no slip methods

dateTime: object of clsDateTime

date: measurement date

startSerialTime: Matlab serial time for start time

endSerialTime: Matlab serial time for end time

transectDuration_sec: duration of transect in seconds

ensDuration_sec: duration of each ensemble in seconds 


\section{Appendix 2. Extensible Markup Language (XML) Output Format.}

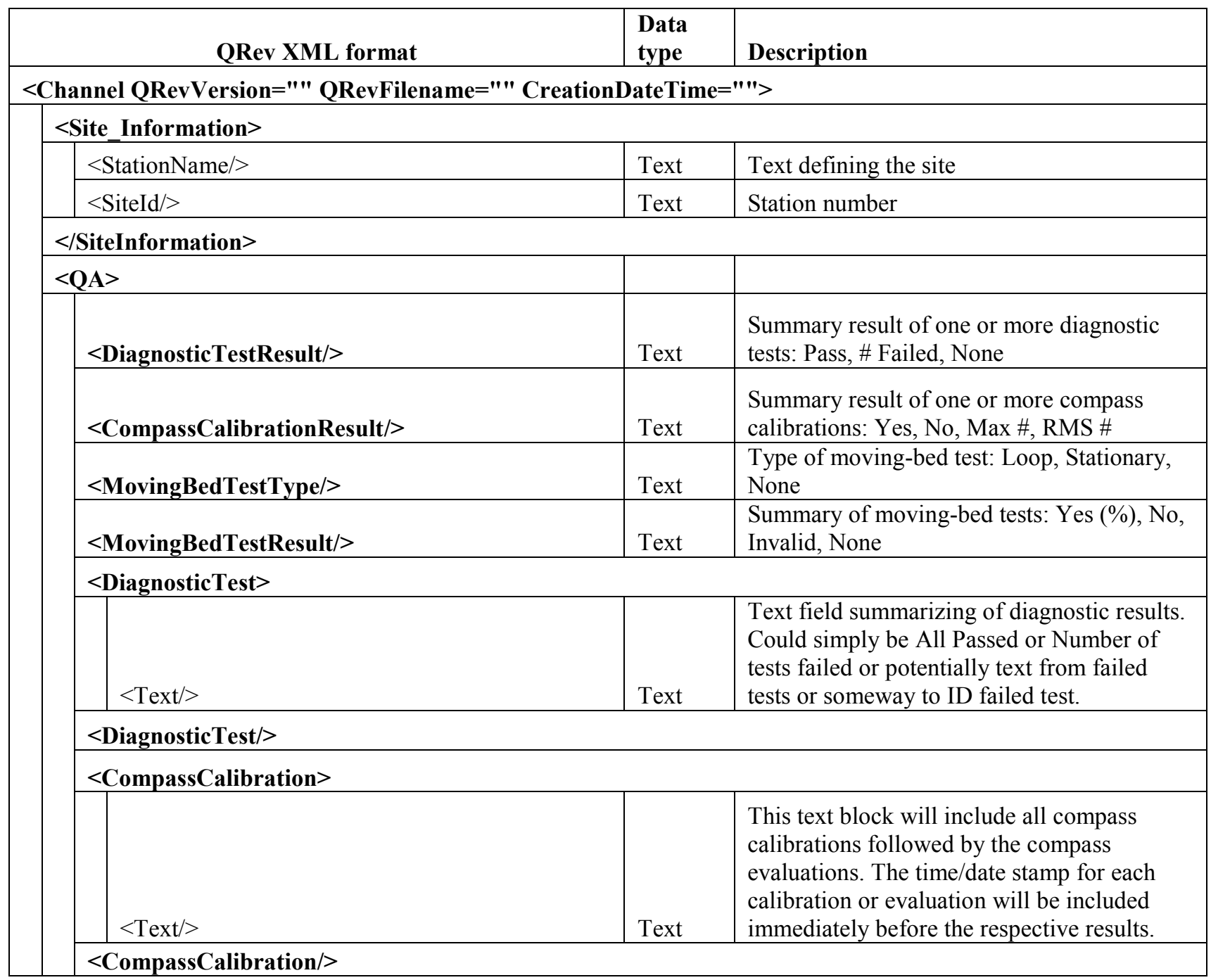




\section{Appendix 2. Extensible Markup Language (XML) Output Format.- Continued}

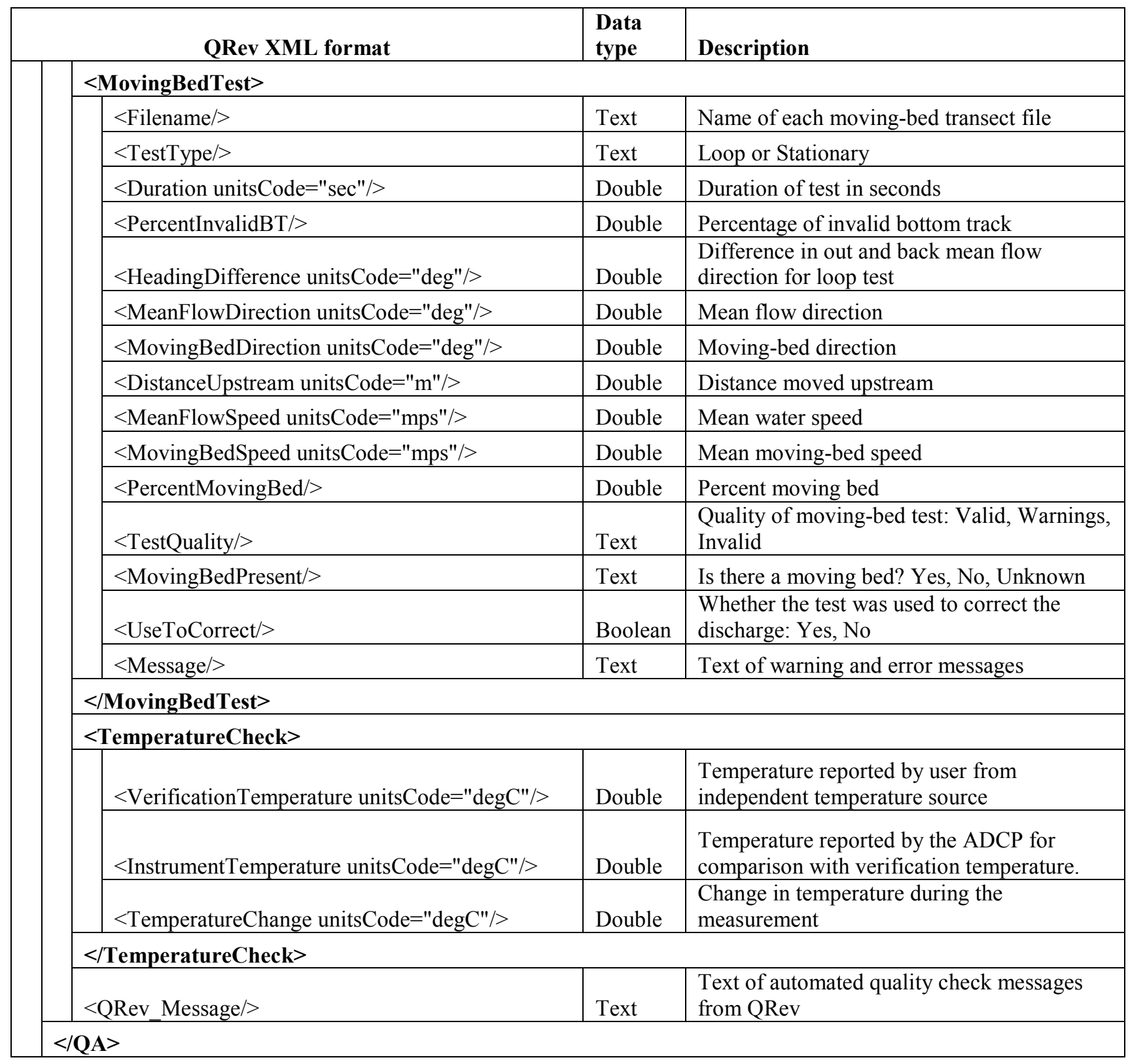




\section{Appendix 2. Extensible Markup Language (XML) Output Format.- Continued}

\begin{tabular}{|c|c|c|}
\hline QRev XML format & $\begin{array}{l}\text { Data } \\
\text { type }\end{array}$ & Description \\
\hline \multicolumn{3}{|l|}{$<$ Instrument $>$} \\
\hline$<$ Manufacturer $/>$ & Text & Manufacturer: TRDI or SonTek \\
\hline$<$ Model $/>$ & Text & $\begin{array}{l}\text { Model: Rio Grande (Freq), StreamPro, } \\
\text { RiverRay, RiverPro, M9, S5 }\end{array}$ \\
\hline$<$ SerialNumber $>$ & Text & Text field for serial number of instrument \\
\hline$<$ FirmwareVersion/> & Text & Text field for firmware version \\
\hline$<$ Frequency unitsCode $=" \mathrm{kHz} / />$ & Text & $\begin{array}{l}\text { Frequency of ADCP in kHz: could be Multi } \\
\text { for M9, S5 }\end{array}$ \\
\hline$<$ BeamAngle unitsCode $=$ "deg" $/>$ & Double & Angle of beams relative to vertical \\
\hline$<$ BlankingDistance unitsCode $=" \mathrm{~m} " />$ & Double & Blanking distance \\
\hline$<$ InstrumentConfiguration $>$ & Text & $\begin{array}{l}\text { Text block containing all commands or other } \\
\text { user settings }\end{array}$ \\
\hline \multicolumn{3}{|l|}{$</$ Instrument $>$} \\
\hline \multicolumn{3}{|l|}{$<$ Processing $>$} \\
\hline$<$ SoftwareVersion/> & Text & Version of software used to process the data \\
\hline$<$ Type $/>$ & Text & Type of processing: TRDI, SonTek, QRev \\
\hline$<$ AreaComputationMethod/> & Text & Method used to compute area: Parallel \\
\hline \multicolumn{3}{|l|}{$<$ Navigation $>$} \\
\hline$<$ Reference/ $>$ & Text & Navigation reference used: BT, GGA, VTG \\
\hline$<$ CompositeTrack $>$ & Text & Use composite tracks: On, Off \\
\hline$<$ MagneticVariation unitsCode="deg" $>$ & Double & Magnetic variation \\
\hline$<$ BeamFilter $/>$ & Text & BeamFilter: 3, 4, Auto \\
\hline$<$ ErrorVelocityFilter unitsCode="mps"/> & Text & $\begin{array}{l}\text { Error velocity filter setting: Auto, Off, or } \\
\text { threshold value }\end{array}$ \\
\hline$<$ VerticalVelocityFilter unitsCode="mps" $>>$ & Text & $\begin{array}{l}\text { Vertical velocity filter setting: Auto, Off, or } \\
\text { threshold value }\end{array}$ \\
\hline$<$ OtherFilter/ $>$ & Text & Other Filter: On, Off \\
\hline$<$ GPSDifferentialQualityFilter/> & Text & Differential Quality Filter: 1, 2, 4 \\
\hline$<$ GPSAltitudeFilter/ $>$ & Text & $\begin{array}{l}\text { Altitude filter: Auto, Off, threshold value for } \\
\text { change in altitude }\end{array}$ \\
\hline$<$ HDOPChangeFilter/> & Text & $\begin{array}{l}\text { HDOP filter: Auto, Off, threshold value for } \\
\text { HDOP change }\end{array}$ \\
\hline$<$ HDOPThresholdFilter/> & Text & $\begin{array}{l}\text { HDOP filter: Auto, Off, threshold value for } \\
\text { maximum HDOP }\end{array}$ \\
\hline$<$ InterpolationType/ $>$ & Text & $\begin{array}{l}\text { Interpolation type: None, Linear, } \\
\text { ExpandedT, Hold9, HoldLast, Smooth }\end{array}$ \\
\hline \multicolumn{3}{|l|}{$<$ /Navigation $>$} \\
\hline
\end{tabular}




\section{Appendix 2. Extensible Markup Language (XML) Output Format.- Continued}

\begin{tabular}{|c|c|c|}
\hline QRev XML format & $\begin{array}{l}\text { Data } \\
\text { type }\end{array}$ & Description \\
\hline \multicolumn{3}{|l|}{$<$ Depth $>$} \\
\hline$<$ Reference $/>$ & Text & Source of depths: BT, VB, DS \\
\hline$<$ CompositeDepth/> & Text & Use composite depths: On, Off \\
\hline$<$ ADCPDepth unitsCode="m"/> & Double & ADCP draft \\
\hline$<$ ADCPDepthConsistent/> & Boolean & All transects have same draft: Yes, No \\
\hline$<$ FilterType/> & Text & Type of depth filter: None, TRDI, Smooth \\
\hline$<$ InterpolationType/> & Text & $\begin{array}{l}\text { Interpolation type: None, Linear, Smooth, } \\
\text { HoldLast }\end{array}$ \\
\hline$<$ AveragingMethod/> & Text & Averaging method for BT: Simple, IDW \\
\hline \multicolumn{3}{|l|}{ </Depth> } \\
\hline \multicolumn{3}{|l|}{$<$ WaterTrack $>$} \\
\hline$<$ ExcludedDistance unitsCode $=" \mathrm{~m} " />$ & Double & $\begin{array}{l}\text { Minimum distance below the transducer in } \\
\text { which cells will be marked invalid. This is } \\
\text { only applicable if it is larger than the blank. }\end{array}$ \\
\hline$<$ BeamFilter $>$ & Text & Beam Filter: 3, 4, Auto \\
\hline$<$ ErrorVelocityFilter unitsCode="mps"/> & Text & $\begin{array}{l}\text { Error velocity filter setting: Auto, Off, or } \\
\text { threshold value }\end{array}$ \\
\hline$<$ VerticalVelocityFilter unitsCode="mps" $/>$ & Text & $\begin{array}{l}\text { Vertical velocity filter setting: Auto, Off, or } \\
\text { threshold value }\end{array}$ \\
\hline$<$ OtherFilter $>$ & Text & Other Filter: On, Off \\
\hline$<$ SNRFilter $>$ & Text & SNR filter setting: Auto, Off \\
\hline$<$ CellInterpolation/> & Text & Interpolate invalid cells: None, TRDI, Linear \\
\hline$<$ EnsembleInterpolation/> & Text & $\begin{array}{l}\text { Interpolate invalid ensembles: None, } \\
\text { ExpandedT, Hold } 9 \text {, HoldLast, Linear, TRDI }\end{array}$ \\
\hline \multicolumn{3}{|l|}{$</$ WaterTrack $>$} \\
\hline \multicolumn{3}{|l|}{$<$ Edge $>$} \\
\hline$<$ RectangularEdgeMethod/ $>$ & Text & $\begin{array}{l}\text { Method used for rectangular edges: TRDI, } \\
\text { SonTek, QRev }\end{array}$ \\
\hline$<$ VelocityMethod/> & Text & $\begin{array}{l}\text { Method used to compute edge velocity: } \\
\text { TRDI, SonTek, QRev }\end{array}$ \\
\hline$<$ LeftType/> & Text & $\begin{array}{l}\text { Left edge type: Triangular, Square, Custom, } \\
\text { User, Varies }\end{array}$ \\
\hline$<$ LeftEdgeCoefficient $>$ & Text & $\begin{array}{l}\text { Coefficient used to compute left edge Q, N/A } \\
\text { if user or Varies for Type }\end{array}$ \\
\hline$<$ RightType $>$ & Text & $\begin{array}{l}\text { Right edge type: Triangular, Square, Custom, } \\
\text { User, Varies }\end{array}$ \\
\hline$<$ RightEdgeCoefficient/> & Text & $\begin{array}{l}\text { Coefficient used to compute right edge Q, } \\
\text { N/A if user or Varies for Type }\end{array}$ \\
\hline \multicolumn{3}{|l|}{$</$ Edge $>$} \\
\hline
\end{tabular}




\section{Appendix 2. Extensible Markup Language (XML) Output Format.- Continued}

\begin{tabular}{|c|c|c|}
\hline QRev XML format & $\begin{array}{l}\text { Data } \\
\text { type }\end{array}$ & Description \\
\hline \multicolumn{3}{|l|}{$<$ Extrapolation $>$} \\
\hline$<$ TopMethod/> & Text & $\begin{array}{l}\text { Top extrapolation method: Constant, Power, } \\
\text { 3-Point }\end{array}$ \\
\hline$<$ BottomMethod/> & Text & $\begin{array}{l}\text { Bottom extrapolation method: Power, No } \\
\text { Slip }\end{array}$ \\
\hline$<$ Exponent $/>$ & Double & Exponent for power and no slip methods \\
\hline \multicolumn{3}{|l|}{$</$ Extrapolation $>$} \\
\hline \multicolumn{3}{|l|}{$<$ Sensor $>$} \\
\hline$<$ TemperatureSource/> & Text & $\begin{array}{l}\text { Source of temperature for computing speed } \\
\text { of sound: ADCP, User, Varies }\end{array}$ \\
\hline$<$ Salinity unitsCode="ppt" $/>$ & Text & Mean salinity for transect: \#, ADCP, Varies \\
\hline$<$ SpeedofSound $/>$ & Text & $\begin{array}{l}\text { Source of speed of sound for transect: } \\
\text { ADCP, Calc, \#, Varies }\end{array}$ \\
\hline \multicolumn{3}{|l|}{$</$ Sensor $>$} \\
\hline \multicolumn{3}{|l|}{$</$ Processing $>$} \\
\hline \multicolumn{3}{|l|}{$<$ Transect $>$} \\
\hline$<$ Filename/ $>$ & Text & Name of each transect file \\
\hline$<$ StartDateTime $/>$ & Date & Start time and date of each transect \\
\hline$<$ EndDateTime/ $>$ & Date & End time and date of each transect \\
\hline \multicolumn{3}{|l|}{$<$ Discharge $>$} \\
\hline$<$ Top unitsCode $=" \mathrm{cms} " />$ & Double & Top discharge \\
\hline$<$ Middle unitsCode $=" \mathrm{cms} " />$ & Double & Middle or measured discharge \\
\hline$<$ Bottom unitsCode $=$ "cms" $/>$ & Double & Bottom discharge \\
\hline$<$ Left unitsCode $=" \mathrm{cms} " />$ & Double & Left edge discharge \\
\hline$<$ Right unitsCode $=" \mathrm{cms} " />$ & Double & Right edge discharge \\
\hline$<$ Total unitsCode="cms"/> & Double & Total discharge for transect \\
\hline$<$ MovingBedPercentCorrection/ $>$ & Double & $\begin{array}{l}\text { Percent correction if a moving-bed correction } \\
\text { was applied }\end{array}$ \\
\hline$</$ Discharge $>$ & & \\
\hline
\end{tabular}




\section{Appendix 2. Extensible Markup Language (XML) Output Format.- Continued}

\begin{tabular}{|c|c|c|}
\hline QRev XML format & $\begin{array}{l}\text { Data } \\
\text { type }\end{array}$ & Description \\
\hline \multicolumn{3}{|l|}{$<$ Edge $>$} \\
\hline$<$ StartEdge $/>$ & Text & Edge where transect started: Left, Right \\
\hline$<$ RectangularEdgeMethod/> & Text & $\begin{array}{l}\text { Method used for rectangular edges: TRDI, } \\
\text { SonTek, QRev }\end{array}$ \\
\hline$<$ VelocityMethod/ $>$ & Text & $\begin{array}{l}\text { Method used to compute edge velocity: } \\
\text { TRDI, SonTek, QRev }\end{array}$ \\
\hline$<$ LeftType/> & Text & $\begin{array}{l}\text { Left edge type: Triangular, Square, Custom, } \\
\text { User }\end{array}$ \\
\hline$<$ LeftEdgeCoefficient $/>$ & Double & Coefficient used to compute left edge Q \\
\hline$<$ LeftUserQ unitsCode="cms" $/>$ & Double & User supplied left edge Q \\
\hline$<$ LeftDistance unitsCode $=" \mathrm{~m} " />$ & Double & Distance from left bank \\
\hline$<$ LeftNumberEnsembles/> & Integer & $\begin{array}{l}\text { Number of valid ensembles used for the left } \\
\text { edge computation }\end{array}$ \\
\hline$<$ RightType/> & Text & $\begin{array}{l}\text { Right edge type: Triangular, Square, Custom, } \\
\text { User }\end{array}$ \\
\hline$<$ RightEdgeCoefficient/> & Double & Coefficient used to compute right edge $\mathrm{Q}$ \\
\hline$<$ RightUserQ unitsCode="cms" $>>$ & Double & User supplied right edge $\mathrm{Q}$ \\
\hline$<$ RightDistance unitsCode $=" \mathrm{~m} " />$ & Double & Distance from right bank \\
\hline$<$ RightNumberEnsembles/> & Integer & $\begin{array}{l}\text { Number of valid ensembles used for the right } \\
\text { edge computation }\end{array}$ \\
\hline \multicolumn{3}{|l|}{$</$ Edge $>$} \\
\hline \multicolumn{3}{|l|}{$<$ Sensor $>$} \\
\hline$<$ TemperatureSource/> & Text & $\begin{array}{l}\text { Source of temperature for computing speed } \\
\text { of sound: ADCP, User }\end{array}$ \\
\hline$<$ MeanTemperature unitsCode $=" \operatorname{degC} " />$ & Double & Mean water temperature for transect \\
\hline$<$ MeanSalinity unitsCode="ppt" $/>$ & Double & Mean salinity for transect \\
\hline$<$ SpeedofSoundSource/> & Text & $\begin{array}{l}\text { Source of speed of sound for transect: } \\
\text { ADCP, Calc, User }\end{array}$ \\
\hline$<$ MeanSpeedofSound unitsCode="mps"/> & Double & Mean speed of sound for transect \\
\hline \multicolumn{3}{|l|}{$</$ Sensor $>$} \\
\hline
\end{tabular}




\section{Appendix 2. Extensible Markup Language (XML) Output Format.- Continued}

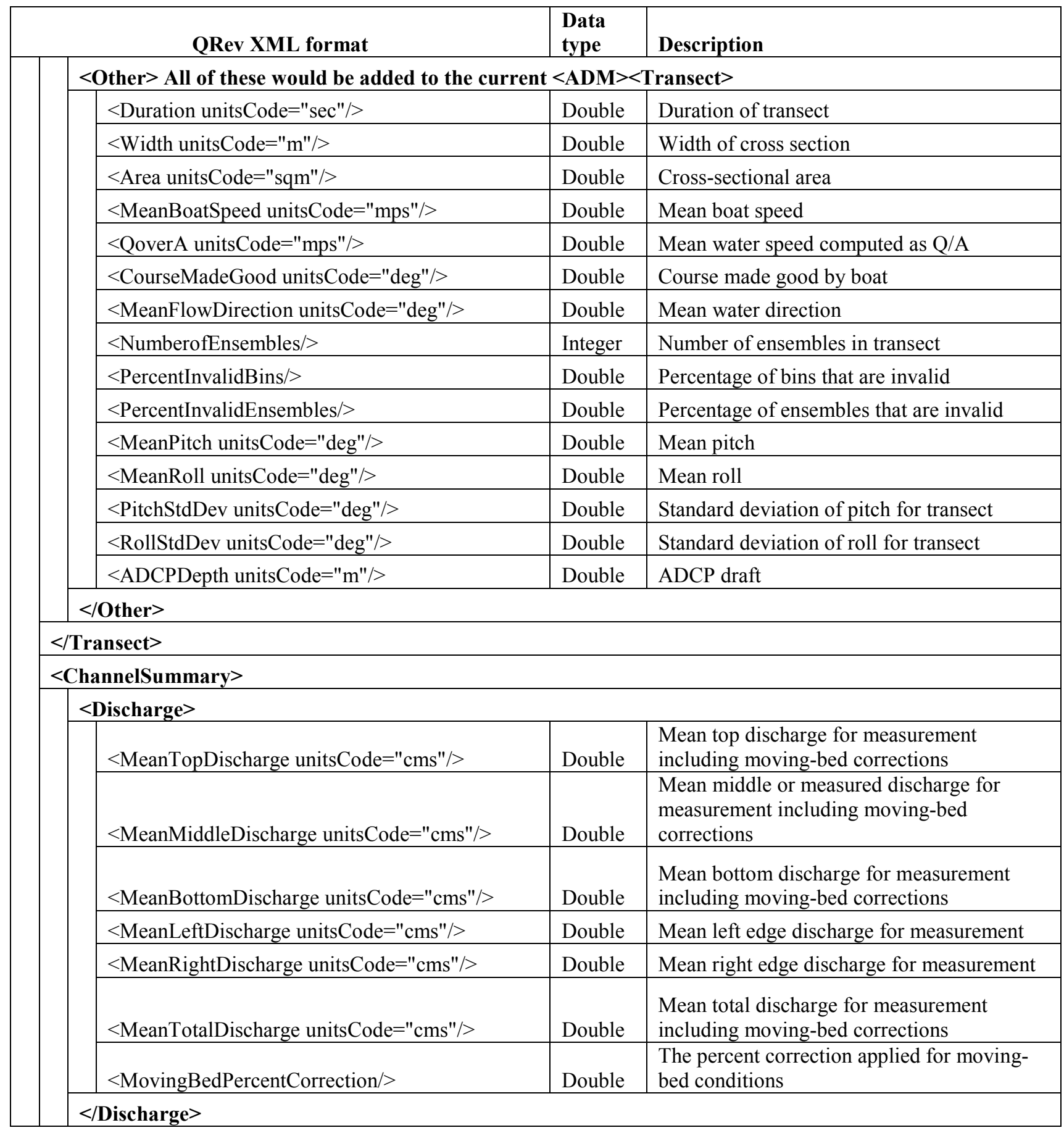




\section{Appendix 2. Extensible Markup Language (XML) Output Format.- Continued}

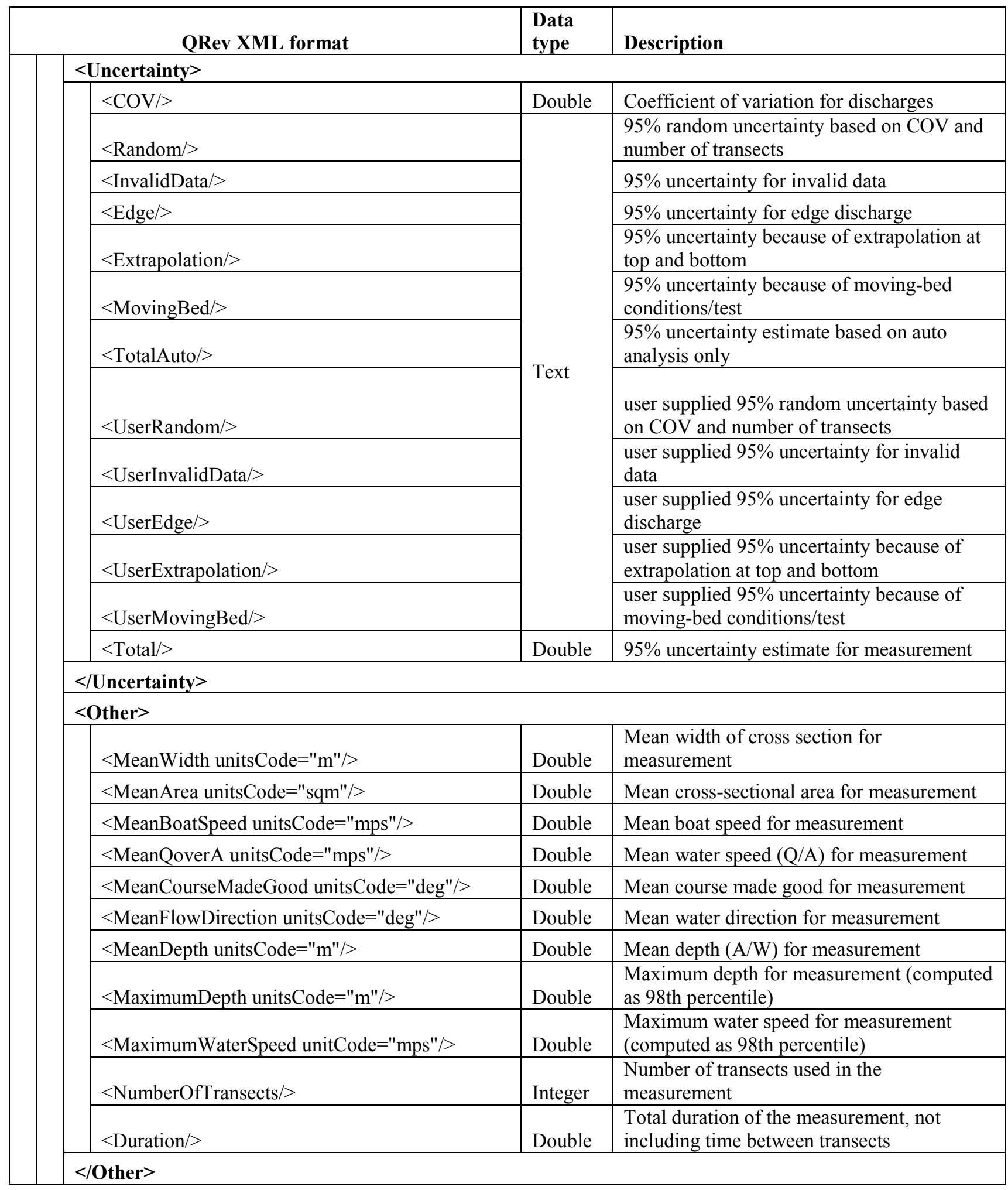




\section{Appendix 2. Extensible Markup Language (XML) Output Format.-}

Continued

\begin{tabular}{|c|c|c|}
\hline QRev XML format & $\begin{array}{l}\text { Data } \\
\text { type }\end{array}$ & Description \\
\hline \multicolumn{3}{|l|}{$</$ ChannelSummary $>$} \\
\hline$<$ UserComment $>$ & Text & $\begin{array}{l}\text { Comments from MMT notes and remarks } \\
\text { and user comments entered into QRev }\end{array}$ \\
\hline
\end{tabular}


For additional information, contact:

Chief, USGS Office of Surface Water

415 National Center

12201 Sunrise Valley Drive

Reston, VA 20192 (703) 648-5301

Or visit the Office of Surface Water Web site at: http://water.usgs.gov/osw/ 
ISSN 2331-1258 (online)

http://dx.doi.org/10.3133/ofr20161052 